CERN / PPE 94-184

November 21, 1994

\title{
QCD Results from the Study of Hadronic $Z$-Decays
}

\author{
Michael Schmelling / CERN
}

\begin{abstract}
The high statistics data collected from hadronic $Z$-decays have lead to remarkable progress in the understanding of the dynamics of strong interactions. In addition to precision measurements of the strong coupling constant, the colour factors defining the gauge structure of QCD were determined with large accuracy. They are found to agree with the expectation for an unbroken SU(3) symmetry, thus establishing unambigously the non-abelian nature of QCD. Higher order effects in many cases are known to next-to-leading logarithmic precision. Coherence effects as predicted in the framework of the modified leading-log approximation and local parton-hadron duality are experimentally well established. Studies of the hadronization process favour a chain-like production mechanism which bridges the gap between perturbative QCD as described by coherent parton shower models and the final state hadrons.
\end{abstract}

Submitted to Physica Scripta 


\section{Introduction}

After the discovery of the partonic structure of hadrons which lead to the quark-parton model, QCD was formulated in analogy to QED as a gauge theory which describes strong interactions between quarks via exchange of massless gauge bosons, the "gluons". Using the knowledge obtained e.g. from hadron spectroscopy [1], the measurement of the $\pi^{0}$ decay rate [2] or the total $e^{+} e^{-}$-annihilation cross section into hadronic final states [3], that quarks have three internal degrees of freedom, it was natural to assume that those degrees of freedom are associated with the charge of QCD, called "colour". The additional requirement that bound states of three quarks or a quark-antiquark pair exist as colour singlets, i.e. without net colour charge, made SU(3) the natural candidate for the gauge group of QCD [4].

An important difference between QED and QCD is that the gauge bosons carry of QCD colourcharge. Gluons thus couple directly to gluons. A consequence is that vacuum polarization effects produce an anti-screening of the bare QCD charges, which results in the strong coupling constant to diverge at large distances and to become small at short distances $[5,6]$. This explains why quarks are not observed as free particles [7] and at the same time renders perturbation theory applicable to describe processes involving large momentum transfers.

The $Z$ produced in $e^{+} e^{-}$-annihilations is not only an ideal laboratory to study electro-weak interactions. It also permits precision measurements of strong interactions by studying QCD corrections to the well defined initial state of a $Z$ decaying into a quark-antiquark pair. The LEP centre-of-mass energy of $91.2 \mathrm{GeV}$ is about three times higher than at PEP/PETRA and about $50 \%$ larger than at TRISTAN. Perturbative QCD predicts corrections which evolve as $1 / \ln \left(E_{c m}\right)$ whereas non-perturbative effects are expected to scale with $1 / E_{c m}$. Thus already the higher energy improves the prospects for precision tests of perturbative QCD. As an added advantage the cross section on the $Z$-resonance is much larger than for any of the machines mentioned above. The energy dependence of the total hadronic cross section as function of the centre-of-mass energy is shown in fig.1. Since the startup of LEP each of the four experiments has collected several million hadronic $Z$ decays which were analyzed for precision measurements of the strong coupling constant, tests of its flavour independence, to probe the gauge structure of QCD and to study coherence effects and the hadron formation mechanism.

Up to gauge fixing terms the Lagrangian of $\mathrm{QCD}, \mathrm{L}_{\mathrm{QCD}}$, is the Yang-Mills Lagrangian for an unbroken $\mathrm{SU}(3)$ gauge symmetry. Figure 2 gives a pictorial representation of $\mathrm{L}_{\mathrm{QCD}}$, showing the free fields and interaction terms together with the factors which determine the relative coupling strengths. Quarks have three and gluons have eight colour degrees of freedom. The amplitude for a quark changing its colour from $i$ to $j$ by emitting a gluon of type $c$ is given by $g \lambda_{c}^{i j} / 2$, i.e. proportional to an unknown gauge coupling $g$ and the element $i j$ of the Gell-Mann matrix $\lambda_{c}$. Similarly, the amplitude for a gluon of type $a$ changing to $b$ by emitting a gluon of type $c$ is given by $g f^{a b c}$, with $f^{a b c}$ the structure constants of SU(3). The existence of the latter kind of coupling $f^{a b c} \neq 0$ is characteristic for a non-abelian theory. Although the gauge symmetry is unbroken, the conceptually simple situation is complicated by the large value of the strong coupling constant which renders perturbative calculations reliable only in the limit of large momentum transfers. In this regime QCD tests can be understood intuitively as probing the diagrams that constitute the terms of $\mathrm{L}_{\mathrm{QCD}}$, focusing on the determination of the coupling constant and the gauge structure of the theory. 


\section{Phenomenology of Hadronic Z decays}

Although not yet amenable to a rigorous mathematical treatment, the production of multihadronic final states in $e^{+} e^{-}$-annihilation processes can be described rather accurately by Monte-Carlo simulations, which combine results from perturbative QCD with phenomenological models for the non-perturbative hadronization process. Starting from an initial quark-antiquark pair those models have to generate final states with an average of around twenty charged and about the same number of neutral particles.

The basic scenario is depicted in fig.3. The inital quarks start radiating gluons, which in turn can radiate further gluons or split into secondary quark-antiquark pairs. That way an initial virtuality of the primary quarks gradually decreases in a parton showering process until it falls below a cutoff, at which point the cascade stops. Then the final state partons are combined into colour neutral hadrons. This hadronization process is described by phenomenological models. Once the primary hadrons are formed, their decay is modeled according to measured branching ratios and lifetimes.

\subsection{The Perturbative Phase}

Various implementations of this scenario are available as Monte-Carlo programs for direct simulation of multihadron events. Here only a brief overview will be given. Detailed discussions of the material presented below can be found in [8] and references therein.

One approach is realized by the "matrix element" models, available for example as the JETSET ME option of the Lund Monte-Carlo [9], where a fixed order QCD calculation is used to describe the perturbative phase by generating partonic final states according to the exact QCD matrix elements. The obvious advantage of those models is, that interference effects are taken into account properly. However, as complete calculations of the differential matrix elements do not exist beyond $\mathrm{O}\left(\alpha_{s}^{2}\right)$, only 2,3 and 4 parton final states can be generated. In addition, MonteCarlo implementations usually introduce a cutoff parameter such, that different sub-processes only contribute in regions of phase space where the finite order calculation of the cross section is positive. Although based on exact matrix elements this cutoff dependence leads to slight deviations from the proper QCD prediction. The average parton multiplicity in those models is typically around 2.7, i.e. the modeling of the non-perturbative phase has to bridge the gap from this to a much larger number of final state particles. Matrix element models work reasonably well up to PEP/PETRA energies. At LEP energies they are outperformed by the parton shower approach.

The parton shower picture for higher order QCD processes is derived from the leading-log approximation, which for collinear emissions can be interpreted as a stochastic process where a parton cascade develops from an initial quark-antiquark state without interference effects between subsequent emissions. It is thus well suited for an implementation as a Monte-Carlo model. The momentum sharing between the daughter partons is determined by the Altarelli-Parisi splitting kernels. The simplest approach to incorporate also transverse momenta into the shower is to assign them independently in each subsequent splitting process, e.g. according to a gaussian probability distribution. This constitutes what is referred to as a "conventional" or "incoherent" 
parton shower model. The most important representative for this class of models is the COJETS program $[10,11,12,13]$.

QCD corrections beyond leading-logarithmic accuracy can be included by certain refinements of this basic scenario. The most important one, taking into account the bulk of the interference effects between subsequent splitting processes in the parton cascade is "angular ordering", i.e. decreasing emission angles as the parton shower proceeds. Models with angular ordering are also referred to as "coherent" parton shower models. Further improvements are obtained by matching the first gluon emission to the exact $\mathrm{O}\left(\alpha_{s}\right)$ matrix element, thereby ensuring that a hard radiation process in the initial phase of the parton shower is properly described by QCD. Nowadays most parton shower models are of the coherent type including the $\mathrm{O}\left(\alpha_{s}\right)$ matching. The currently most widely used generator with these features is the JETSET PS model $[14,15,16]$.

Additional refinements of the parton-shower picture are possible. The HERWIG generator [17, 18, 19] e.g. includes inter-jet interference and gluon polarization effects. In the NLLJET program $[20,21]$ the leading-order Altarelli-Parisi splitting kernels are replaced by the next-toleading order ones, which contain the $\mathrm{O}\left(\alpha_{s}^{2}\right)$ corrections to the $2 \rightarrow 2$ splitting functions and describe also $2 \rightarrow 3$ splitting processes. ARIADNE $[22,23,24]$ implements an alternative way to formulate the parton showering process as colour-dipole radiation which includes many of the non-trivial effects automatically. Here the initial quark-antiquark pair is considered as a colourdipole that radiates a gluon according to the leading-oder QCD matrix element. This gluon splits the initial dipole into two secondary ones which can radiate in turn. Iterating this process gives rise to a parton shower, which automatically includes the matching to the $\mathrm{O}\left(\alpha_{s}\right)$ matrix element, angular ordering and some of the azimuthal correlations between jets.

The angular ordering property of higher order QCD corrections has an important phenomenological implication as it predicts that the energy flow in the shower remains collimated around the direction of the initial partons. This explains why most hadronic $Z$ decays exhibit a pronounced 2-jet structure, i.e. a topology where most of the energy of the final state particles is emitted into rather narrow back-to-back angular regions. Furthermore, if in the initial stage of the parton shower a hard gluon is emitted at a large angle, it gives rise to a well separated jet of its own. Intuitively jets thus can be interpreted as the hard partonic skeleton of a hadronic $Z$ decay.

\subsection{The Hadronization Process}

Phenomenological models are employed to describe the hadronization process, i.e. the nonperturbative conversion of a partonic configuration into final state hadrons. Historically first was the "independent fragmentation" ansatz, where each parton gives rise to its own jet of hadrons. It can be characterized as a simple iterative procedure, where an inital quark picks up an antiquark from a vacuum fluctuation to form a meson leaving behind the other quark. The sharing of momentum between meson and remaining quark is described by a "fragmentation function" $f(z)$, which is the probability density for the meson to carry a fraction $z$ of the initial quark momentum.

The leftover quark carries the momentum fraction $1-z$. Transverse momentum components are introduced e.g. according to a gaussian distribution with zero mean and a standard deviation of typically $300 \mathrm{MeV}$. The procedure is iterated with the remaining quark, until the energy falls 
below a cutoff. Gluons can be treated by splitting them into an quark-antiquark pair first and then going through the same mechanism for hadron production. A refinement of this scheme is realized in the COJETS Monte-Carlo model.

Another approach is given in the framework of the LUND string model $[25,26]$. Here the nonperturbative interaction between partonic colour charges and the formation of the final state hadrons is modeled by the breakup of a colour flux tube. This ansatz is motivated by the observation, that due to the gluon self-coupling the field lines of a static colour field attract each other to form a flux tube. Assuming that this "string" has constant energy per unit length one expects the QCD potential to grow linearly at large distances. Such a long distance behaviour is supported by lattice calculations and provides a natural explanation for quark confinement. In the string fragmentation model a string is stretched from a quark to an antiquark. Gluons, which have the colour structure of an quark-antiquark state, can be viewed as simultaneously terminating one and starting another string, i.e. they act as kinks in the original string. As the partons move apart, more and more energy goes into the string until it breaks by the production of a new quarkantiquark pair, which terminates the resulting daughter-strings. At large enough energies further breaks of the daughter-strings occur until only on-shell hadrons remain, each hadron corresponding to a small piece of string. Again the breakup of the string is governed by a fragmentation function. Interpreting the breakup mechanism as a tunneling phenomenon and treating different breakups to be causally disconnected fixes the functional form of the fragmentation function, leaving only two (highly correlated) free parameters. Furthermore, the finite transverse dimensions of the string provide a natural scale for the creation of transverse momenta.

The last model to be mentioned here is the cluster fragmentation model. Here the gluons at the end of the perturbative phase are split into quark-antiquark pairs. Colour-neutral pairs of quarks that are close in phase space then recombine into massive clusters which decay isotropically into observable hadrons.

The cluster fragmentation scheme is attractive as no explicit assumptions about fragmentation functions and the generation of transverse momenta are needed. The Lund-string model on the other hand, based on a QCD inspired concept, has the potential to account also for genuine QCDeffects in the transition region between the perturbative and the non-perturbative phase if the free parameters of the fragmentation function are suitably adjusted. The basic ideas of the two models are depicted in fig.4.

\subsection{Comparison with Data}

The concepts outlined in the previous sections are realized in various Monte-Carlo programs. The most important ones with their key ingredients are listed in table 1 . In addition to the parameters given in the table there are many others which fine tune the properties of the generator. The LEP-collaborations performed parameter adjustments in order to optimize the agreement between data and Monte-Carlo. Results are collected in the appendix. Figures 5,7,6,8 illustrate the level of agreement that has been reached for a few characteristic distributions.

One of the simplest quantities to describe the properties of multihadron events is the charged particle multiplicity distribution. Figure 5 shows a measurement compared to various model calculations, where some really give an excellent description of the experimental data. Also the 


\begin{tabular}{|l|c|c|c|c|c|c|}
\hline & JETSET PS & HERWIG & ARIADNE & NLLJET & COJETS & JETSET ME \\
\hline Parton Level & coherent PS & coherent PS & colour dipole & coherent PS & LL - PS & O $\left(\alpha_{s}^{2}\right) \mathrm{ME}$ \\
Splitting & $\mathrm{LL}$ & $\mathrm{LL}$ & $\mathrm{O}\left(\alpha_{s}\right) \mathrm{ME}$ & $\mathrm{NLL}$ & $\mathrm{LL}$ & - \\
First Gluon & $\mathrm{O}\left(\alpha_{s}\right)$ & $\mathrm{O}\left(\alpha_{s}\right)$ & $\mathrm{O}\left(\alpha_{s}\right)$ & $\mathrm{O}\left(\alpha_{s}^{2}\right)$ & $\mathrm{O}\left(\alpha_{s}\right)$ & - \\
Ang. Ordering & yes & yes & yes & yes & no & - \\
Jet-Jet correl. & no & yes & yes & no & no & yes \\
Parameters & $\Lambda_{\mathrm{LL}}, Q_{0}$ & $\Lambda_{\mathrm{LL}}, M_{g}$ & $\Lambda_{\mathrm{LL}}, P_{\mathrm{min}}^{\mathrm{T}}$ & $\Lambda_{\mathrm{NLL}}, Q_{0}$ & $\Lambda_{\mathrm{LL}}, Q_{0}$ & $\Lambda_{\mathrm{Mf}}^{\mathrm{eff}}, f, y_{\min }$ \\
Typical values & $0.4,1.0$ & $0.18,0.75$ & $0.2,1.0$ & $0.2,1.0$ & $\mathbf{0 . 1 7}, 3.0$ & $\mathbf{0 . 5 , 0 . 0 0 2 , 0 . 0 2}$ \\
$\langle \#$ partons $\rangle$ & 11.3 & 6.2 & 5.8 & 5.4 & 4.4 & 2.7 \\
\hline Hadronization & String & Cluster & String & String & Independent & String \\
Parameters & $\sigma, \mathrm{B}$ & $M_{\max }$ & $\sigma, \mathrm{B}$ & $\sigma, \mathrm{B}$ & $(\sigma, b, d)_{q, g}$ & $\sigma, \mathrm{B}$ \\
\hline
\end{tabular}

Table 1: Characteristics of the most common Monte-Carlo models. The notations PS, ME, LL and NLL stand for Parton Shower, Matrix Element, Leading-Logarithmic and Next-to-Leading Logarithmic, respectively. The upper part of the table refers to the perturbative phase, the lower one to the hadronization stage. For a detailed explanation of the meaning of the individual parameters see [8] and references therein. Results from parameter adjustments are collected in the appendix. The numbers given above apply for the default versions of JETSET 7.3, HERWIG 5.6, ARIADNE 4.02, NLLJET 2.0 and COJETS 6.22. All except the Herwig model also allow to use the Peterson et al. [27] fragmentation functions for the heavy quarks, adding two additional parameters $\varepsilon_{\mathbf{c}}$ and $\varepsilon_{\mathrm{b}}$.

momentum sharing between the final state particles fig.6 is well reproduced. An important global property is the collimation of the momentum flow in an event, measured by the variable "Thrust" $T[30]$ :

$$
T=\max _{\vec{n}} \sum_{p} \frac{|\vec{p} \cdot \vec{n}|}{P_{\text {tot }}} \quad \text { with } \quad \vec{n}^{2}=1 \quad \text { and } \quad P_{\text {tot }}=\sum_{p}|\vec{p}|
$$

An ideal 2-jet event has $T=1$, a perfectly isotropic one has $T=1 / 2$. The "Thrust axis" $\vec{n}$ is the direction along which the momentum flow is maximal. It thus defines a natural event axis, which can be taken as an estimator for the direction of flight of the initial quark-antiquark pair. The Thrust distribution is shown in fig.7. Also here the models successfully reproduce the experimental data. Figure 8 finally demonstrates that also the jet production rates are modeled correctly.

The parton shower models tuned for a centre-of-mass energy of $91.2 \mathrm{GeV}$ not only describe the data at that particular energy rather well, they also extrapolate successfully to lower energies. The matrix-element models are competitive only if the parameter settings are readjusted with varying centre-of-mass energies. This is partly due to the fact that the cutoff parameter which separates the perturbative and the non-perturbative phase is expressed as a constant fraction of the total centre-of-mass energy $y_{\min }$ whereas the parton shower models cut the perturbative evolution at a fixed energy scale $Q_{0}$. 


\section{Parton Spins}

The simplest consistency check of QCD is to verify that the partons have the spin assignments according to $\mathrm{L}_{\mathrm{QCD}}$. Although demonstrated convincingly already at lower energies in [31, 32] for quarks and in $[33,34,35,36]$ for gluons, respectively, this test was also done by the LEP collaborations.

That quarks are spin-1/2 particles can be seen from the angular distribution of the Thrust axis, which reflects the direction of the primary quarks that couple to the $Z$. From simple angularmomentum considerations the angular distribution for two spin- $1 / 2$ fermions from the decay of a spin-1 particle $(Z)$ is given by $1+\cos ^{2} \Theta$, where $\Theta$ denotes the angle to the beam direction. Figure 9 shows the uncorrected angular distribution of the Thrust axis seen in the ALPEH detector compared to a Monte-Carlo calculation which includes the full detector simulation. The data are in perfect agreement with the spin-1/2 assignment for the quarks. The sensitivity to the quark spin is illustrated by comparing the measurements to the expectation from a spin- 0 assignment, which is clearly excluded.

Studies of the gluon spin are published by all four LEP experiments. Information about the spin of the gluon can be obtained from internal correlations in 3 -jet events $[37,38,39]$ or the angular distribution of the event plane as function of the Thrust [40]. The sensitivity of those observables can be gauged by comparing the expectation from the spin-1 vector gluon against a toy model where the gluon is a scalar higgs-like particle. The sensitivity of the differential $q \bar{q} g$ cross-section to the spin of the gluon arises from the fact that a scalar gluon induces a helicity flip for the fermion from which it is emitted, while the vector gluon coupling is helicity conserving.

Correlations inside 3 -jet events can be studied by analyzing the differential cross-section as function of the scaled jet energies $x_{i}$ :

$$
x_{i}=\frac{2 E_{i}}{\sqrt{s}} \quad, \quad x_{1}>x_{2}>x_{3} \quad, \quad x_{1}+x_{2}+x_{3}=2 .
$$

On the $Z$-resonance the leading order cross-sections for the vector and the scalar gluon hypothesis, ignoring mass effects, are given by $[41,39]$

$$
\frac{d^{2} \sigma^{V}}{d x_{1} d x_{2}} \sim \frac{x_{1}^{3}+x_{2}^{3}+x_{3}^{3}}{\left(1-x_{1}\right)\left(1-x_{2}\right)\left(1-x_{3}\right)}
$$

and

$$
\frac{d^{2} \sigma^{S}}{d x_{1} d x_{2}} \sim \frac{x_{1}^{2}\left(1-x_{1}\right)+x_{2}^{2}\left(1-x_{2}\right)+x_{3}^{2}\left(1-x_{3}\right)}{\left(1-x_{1}\right)\left(1-x_{2}\right)\left(1-x_{3}\right)}-10 \frac{\sum c_{a}^{2}}{\sum c_{a}^{2}+c_{v}^{2}}
$$

respectively. The coefficients $c_{v}$ and $c_{a}$ are the vector and axial couplings of the quarks to the $Z$ where the sums run over all active quark flavours. In both cases the differential cross section is singular for $x_{3} \rightarrow 0$, proportional to $1 / x_{3}^{2}$ for the vector gluon and proportional $1 / x_{3}$ for the scalar gluon. The cross section for the latter is less singular, because the spin flip induced by the emission of a soft scalar gluon results in a final state with antiparallel spins for the two fermions, which has less overlap with the vector state of the initial $Z$-boson.

The difference in the singularity structure is probed e.g. by the $x_{3}$-projection or by the EllisKarliner angle $\lambda_{\mathrm{EK}}[42]$, the angle between the highest energy jet and the two lower energy 
ones in the rest-frame of the two lower energy jets. It is related to the scaled jet-energies via $\cos \lambda_{\mathrm{EK}}=\left(x_{2}-x_{3}\right) / x_{1}$. Figure 10 shows measurements by the L3-collaboration compared to the expectation for a vector and a scalar gluon. The data are well described by the more singular behaviour expected for the vector gluon hypothesis and are in clear disagreement with the scalar gluon model.

The sensitivity of the orientation of the event plane to the spin of the gluon is a particular feature of the different vector and axial-vector couplings of the $Z$ to the primary quarks [43, 44]. The angular distribution of the normal vector of the event plane with respect to the direction of the incoming beams can be described by

$$
\frac{d \sigma}{d \cos \Theta_{n}} \sim 1+a(T) \cos ^{2} \Theta_{n}
$$

with an asymmetry parameter $a(T)$, which in general is a function of the Thrust $T$ of the event. For vector gluons the prediction is $a(T)_{V} \equiv-1 / 3$, for the scalar gluon $a(T)$ increases with decreasing values of $T$. Experimental results from the DELPHI collaboration [40] are found to be in good agreement with the vector gluon hypothesis while the scalar gluon model can be ruled out.

\section{Measurements of the Strong Coupling Constant}

Much effort has gone into precise measurements of the strong coupling constant $\alpha_{s}$ at LEP and elsewhere, in order to test that the same universal flavour independent coupling is involved in all strong interaction processes.

Determinations of the strong coupling constant based on perturbative QCD yield a measurement $\alpha_{s}(\mu)$ at a renormalization point $\mu$ which should be close to the natural energy scale $Q$ of the process under study. A priory the choice of $\mu$ is arbitrary, but at finite order the theoretical prediction is most reliable for $\mu \approx Q$, because very different scales $\mu$ and $Q$ result in $\alpha_{s} \ln \left(\mu^{2} / Q^{2}\right)$ as the effective expansion parameter of the perturbative series instead of $\alpha_{s}$. The numerical value obtained by an $\alpha_{s}$-measurement in general depends on the choice of the renormalization scheme. The most common scheme for higher order calculations is the modifiedminimal subtraction ( $\overline{\mathrm{MS}})$ scheme and the implicit convention has become to assume that this scheme has been used if not specified otherwise, i.e. $\alpha_{s}=\alpha_{s} \overline{\mathrm{MS}}$.

The renormalization scale dependence of $\alpha_{s}$ is controlled by the beta-function

$$
\mu \frac{d \alpha_{s}(\mu)}{d \mu}=-\frac{\beta_{0}}{2 \pi} \alpha_{s}^{2}(\mu)-\frac{\beta_{1}}{8 \pi^{2}} \alpha_{s}^{3}(\mu)-\ldots
$$

with

$$
\beta_{0}=11-\frac{2}{3} n_{f} \quad \text { and } \quad \beta_{1}=102-\frac{38}{3} n_{f}
$$

and $n_{f}$ the number of active quark flavours. These first two coefficients of the beta-function are independent of the renormalization prescription. Measurements of $\alpha_{s}$ obtained at different renormalization points can be compared by using eq.(2) to evolve all measurements to a common reference scale. With the advent of LEP this reference scale has become the $Z$-mass, $M_{Z}$. Another 
way of comparing measurements is related to the observation that $\alpha_{s}(\mu)$ becomes infinite at a certain point when evolving towards lower energies, signaling the breakdown of perturbation theory below a certain energy scale. Different measurements thus also can be compared by giving the scale $\Lambda_{\overline{\mathrm{MS}}}^{\left(n_{f}\right)}$ where $\alpha_{s} \overline{\mathrm{MS}}$ diverges in an evolution with $n_{f}$ quark flavours.

In principle both ways of comparing experimental results are equivalent. However, since the integral of eq.(2) cannot be expressed in terms of elementary functions in closed form, approximate formulae which differ in $\mathrm{O}\left(\alpha_{s}^{4}\right)$ usually are employed to relate $\alpha_{s}(\mu), \alpha_{s}\left(M_{Z}\right)$ and $\Lambda_{\overline{\mathrm{MS}}}$. In many cases the resulting differences are small, except when very small energy scales are involved. An expression which directly relates the strong coupling constant at two different scales is given by

$$
\alpha_{s}\left(M_{Z}\right)=\frac{\alpha_{s}(\mu)}{w}\left(1-\frac{\beta_{1}}{\beta_{0}} \frac{\alpha_{s}(\mu)}{4 \pi} \frac{\ln w}{w}\right) \quad \text { with } \quad w=1-\beta_{0} \frac{\alpha_{s}(\mu)}{4 \pi} \ln \frac{\mu^{2}}{M_{Z}{ }^{2}}
$$

This formula is exact to leading and next-to-leading logarithmic accuracy, i.e. it contains all terms and only those terms of the full solution which are of the type $\alpha_{s}^{n}(\mu) \ln ^{m}\left(\mu^{2} / Q^{2}\right)$ with $m=n-1, n-2$. The scale $\Lambda_{\overline{\mathrm{MS}}}$ usually is defined implicitly by

$$
\alpha_{s}(\mu)=\frac{4 \pi}{\beta_{0} L}\left(1-\frac{\beta_{1}}{\beta_{0}^{2}} \frac{\ln L}{L}\right) \quad \text { with } \quad L=\ln \frac{\mu^{2}}{\Lambda_{\overline{\mathrm{MS}}}^{2}} .
$$

Neither of the two expressions is an exact solution to eq.(2).

Comparison of $\alpha_{s}$-measurements at different energy scales often also involves comparing different numbers of active flavours. The evolution thus has to be performed over flavour thresholds. To the accuracy discussed here this is done by keeping the strong coupling $\alpha_{s}$ continuous at the flavour thresholds $\mu_{t h}=m_{q}$ while having discontinous parameters $\beta_{0}, \beta_{1}$

and $\Lambda_{\overline{\mathrm{MS}}}$. Further details concerning the energy-dependence of the strong coupling are discussed in $[45,46]$.

\section{1 $\alpha_{s}$ from $R_{Z}$}

The conceptually simplest measurement of $\alpha_{s}\left(M_{Z}\right)$ is obtained from $R_{Z}$, the ratio of the hadronic to the electronic width of the $Z$

$$
R_{Z}=\frac{\Gamma(Z \rightarrow \text { hadrons })}{\Gamma(Z \rightarrow \text { electrons })}=R_{Z}^{E W}\left(1+\delta_{Q C D}\right)
$$

The value of $R_{Z}$ is dominated by the bare electro-weak couplings of the $Z$ to the final state fermions. The sensitivity to $\alpha_{s}$ is due to the fact that QCD-corrections open up additional final states, thereby increasing the hadronic partial width of the $Z$. The measurement is fully inclusive. The perturbative prediction of the QCD correction has been calculated to $\mathrm{O}\left(\alpha_{s}^{3}\right)$. Nonperturbative effects on a measurement of $\alpha_{s}$ are expected to be of the order $\left(\Lambda / M_{Z}\right)^{2}$, which is negligible. Taking mass effects and electro-weak corrections into account the theoretical prediction can be parametrized as $[47]$

$$
R_{Z}=19.937 \cdot\left(1+1.060 a+0.85 a^{2}-15 a^{3}\right) \quad \text { with } \quad a=\frac{\alpha_{s}\left(M_{Z}\right)}{\pi} .
$$


This expression assumes a Higgs mass of $300 \mathrm{GeV} / c^{2}$ and a top mass of $174 \mathrm{GeV} / c^{2}$ [48]. The theoretical uncertainty of $\alpha_{s}\left(M_{Z}\right)$ derived from $R_{Z}$ is estimated to be

$$
\delta\left(\alpha_{s}\left(M_{Z}\right)\right)= \pm 0.002(\mathrm{QCD}) \pm 0.002(\text { electro weak }) \pm 0.002\left(m_{\text {Higgs }}\right) \pm 0.001\left(m_{\text {top }}\right) .
$$

The QCD-uncertainty is dominated by the renormalization scale dependence of the prediction for $R_{Z}$, the electro-weak error by factorization versus non-factorization assumptions for the QED and QCD corrections. The Higgs mass was varied between $60 \mathrm{GeV} / c^{2}$ and $1000 \mathrm{GeV} / c^{2}$, the uncertainty due to the top mass corresponds to an error $\delta\left(m_{\text {top }}\right)= \pm 16 \mathrm{GeV} / c^{2}$. With the latest LEP average [49], $R_{Z}=20.795 \pm 0.040$, one obtains

$$
\alpha_{s}\left(M_{Z}\right)=0.126 \pm 0.006(\exp ) \pm 0.004(\text { theo })=0.126 \pm 0.007
$$

The largest uncertainty still comes from the experimental error on $R_{Z}$. The combined uncertainties related to the electro-weak sector are next, and only third largest is the genuine QCD error.

\section{2 $\alpha_{s}$ from Event Shape Variables}

\subsubsection{Event Shape Variables}

A measurement of the strong coupling constant from event-shape variables is based on the idea that to leading order the ratio of the 3 -jet and the 2 -jet cross section is proportional to $\alpha_{s}$. To exploit this concept one needs to define variables which are sensitive to the topology of multijet events. Those variables have to be "infrared" and "collinear" safe in order to have them defined in perturbation theory, i.e. they must not change in the limit that the energy of an additional soft gluon goes to zero or if any of the final state momenta is split into two collinear ones.

Various observables satisfying the above criteria have been defined. Central to many subsequent definitions is Thrust as already defined in eq.(1). An analogous analysis of the momentum flow in the plane orthogonal to the Thrust axis $\vec{n}$ defines a quantity "Major-value" $M$ and a second event axis $\vec{m}$. The two vectors $\vec{n}$ and $\vec{m}$ span the event plane. The momentum flow out of this plane is a third event shape variable "Minor value" $Q$.

$$
M=\max _{\vec{m} \perp \vec{n}} \sum_{p} \frac{|\vec{p} \cdot \vec{m}|}{P_{\text {tot }}} \quad \text { with } \quad \vec{m}^{2}=1 \quad \text { and } \quad Q=\sum_{p} \frac{|\vec{p} \cdot(\vec{n} \times \vec{m})|}{P_{\text {tot }}} .
$$

From those observables "Oblateness" [50] is obtained as

$$
O=M-Q
$$

Dividing an event into two hemispheres "+" and "-" by the plane orthogonal to the Thrust axis, event-shape variables can be derived from the invariant hemisphere-masses. Normalizing to the centre-of-mass energy one defines "heavy jet-mass" $M_{\mathrm{H}, \mathrm{T}}$ and "mass difference" $M_{\mathrm{D}, \mathrm{T}}[51]$ by

$$
M_{\mathrm{H}, \mathrm{T}}=\frac{\max \left(m_{+}^{2}, m_{-}^{2}\right)}{s} \quad \text { and } \quad M_{\mathrm{D}, \mathrm{T}}=\frac{\left|m_{+}^{2}-m_{-}^{2}\right|}{s} .
$$


The index $T$ serves as a reminder of the fact, that the hemispheres are defined by the Thrust axis. An alternative definition exists where the division into hemispheres is done such, that the difference between the two hemisphere masses becomes maximal. The corresponding event-shape variables are denoted by $M_{\mathrm{H}, \mathrm{m}}$ and $M_{\mathrm{D}, \mathrm{m}}$.

Other variables probing the transverse structure of an event are jet broadening measures, the "total jet broadening" $B_{t o t}$ and the "wide jet broadening" $B_{w}[52]$. Based on the total transverse momenta $P_{ \pm}^{T}$ measured in the \pm -hemispheres with respect to the Thrust axis, the definitions are

$$
B_{\text {tot }}=\frac{P_{+}^{T}+P_{-}^{T}}{2 P_{\text {tot }}} \text { and } B_{w}=\frac{\max \left(P_{+}^{T}, P_{-}^{T}\right)}{2 P_{\text {tot }}} \quad \text { with } \quad P_{ \pm}^{T}=\sum_{p \in \pm}|\vec{p} \times \vec{n}| .
$$

An alternative variable to measure the collimation of the total momentum flow is the " $\mathrm{C}$ parameter", defined through the quadratic invariant of the momentum tensor $T_{i j}[53,54]$ as

$$
C=3\left(T_{11} T_{22}+T_{22} T_{33}+T_{33} T_{11}-T_{12}^{2}-T_{23}^{2}-T_{31}^{2}\right) \quad \text { with } \quad T_{\alpha \beta}=\frac{1}{P_{t o t}}\left(\sum_{p} \frac{p_{\alpha} p_{\beta}}{|\vec{p}|}\right) .
$$

The indices $\alpha$ and $\beta$ refer to the cartesian components of the momentum vectors. For a perfect 2 -jet event one has $C=0$, for an isotropic event $C=1$.

The observables discussed so far describe the event topology by one global number per event. An alternative approach is pursued by the energy-energy-correlation function [55] EEC, defined by

$$
\operatorname{EEC}(\Theta)=\frac{1}{N_{E V}} \sum_{E V}\left(\sum_{i j} \frac{E_{i} E_{j}}{E_{v i s}^{2}} \delta\left(\Theta-\Theta_{i j}\right)\right) .
$$

The sum runs over all events and inside each event over all pairs $i j$ of final state particles. Each event contributes with the energy-weighted distribution of the opening angles $\Theta_{i j}$ between all pairs. Experimental distributions are integrals of the above expression over finite angular intervals. For ideal 2-jet events $\operatorname{EEC}(\Theta)$ consists only of two spikes, one at $\Theta=0$ and the other one at $\Theta=\pi$. Multijet production fills the gap in between, i.e. the cross section there is sensitive to the strong coupling constant. The 2 -jet component can be removed by studying the energy-energy-correlation asymmetry AEEC

$$
\operatorname{AEEC}(\Theta)=\operatorname{EEC}(\Theta)-\operatorname{EEC}(\pi-\Theta)
$$

A related quantity is the planar triple energy correlation function PTEC. Conceptually similar to the EEC, it is defined as the two-dimensional energy wheighted histogram of the angles between planar triplets of particles within an event [56].

All quantities introduced above are defined directly in terms of the final state particles. On the experimental side this means the final state hadrons, for the theoretical prediction it refers to the final state partons. As the respective multiplicities are rather different non-perturbative effects can lead to considerable uncertainties in an $\alpha_{s}$ measurement. The situation can be improved by reconstructing the partonic skeleton, the jets, of an event before comparing to the theoretical prediction. Aside from removing part of the uncertainties related to the hadronization phase this approach is also the more intuitive one, because it is closer to the basic idea of an $\alpha_{s}$ measurements based on event topologies. 
For a quantitative analysis a proper definition of a "jet" is required, which must be applicable both on parton and on hadron level. Two basic concepts of jet-definition exist. A "cone-based" definition [57], where a jet is defined by a certain minimum energy deposited in a fixed angular cone, or a "cluster-based" definition [58] where jets are understood as the combined momentum of neighbouring tracks.

Cone-based definitions are mainly used for the calorimetric jet-studies done at hadron colliders. An exception is an analysis by the OPAL collaboration [59], where the strong coupling constant is determined from $D_{2}(R)$ and $D_{2}(E)$, the change of the 2-jet rate with cone size and minimum energy inside the cone, respectively.

Most jet analyses at $e^{+} e^{-}$-colliders are based on the cluster approach. Here jets are formed by iteratively combining the closest 4 -vectors, based on a distance measure ("metric") $y_{i j}$, which quantifies the phase-space distance of two 4-momenta $p_{i}$ and $p_{j}$, and a recombination scheme that defines how to merge two 4-momenta into a single one. There is a certain freedom in defining a metric and a recombination scheme. The most common choices for the metric are the invariantmass metric $(M)$, the JADE-metric $(J)$ and the Durham-metric $(D)$ :

$$
y_{i j}^{M}=\frac{\left(p_{i}+p_{j}\right)^{2}}{s}, y_{i j}^{J}=\frac{2 E_{i} E_{j}}{s}\left(1-\cos \Theta_{i j}\right) \text { and } y_{i j}^{D}=\frac{2 \min \left(E_{i}^{2}, E_{j}^{2}\right)}{s}\left(1-\cos \Theta_{i j}\right)
$$

In practical applications the centre-of-mass energy $\sqrt{s}$ usually is replaced by the total visible energy $E_{v i s}$ in order to partly correct for detector effects. For massless particles JADE- and invariantmass metric are equivalent. For particles of the same energy the Durham-metric is identical to the JADE-metric. For small opening angles $\Theta_{i j}$ it is proportional to the square of the transverse momentum of the two particles with respect to the resultant direction of flight. Theoretically the Durham-metric is the preferred choice, since it yields a jet-definition for which the perturbative prediction can be resummed to all orders to leading and next-to-leading logarithmic accuracy. Further details are discussed in reference [60].

The most important recombination schemes are the so called $\mathrm{E}, \mathrm{E} 0$ and $\mathrm{P}$ schemes, where the combination of two 4 -vectors $p_{i}$ and $p_{j}$ into one 4 -vector $p_{i j}$ is defined by

$$
\mathrm{E}:\left(\begin{array}{c}
E_{i j}=E_{i}+E_{j} \\
\vec{p}_{i j}=\vec{p}_{i}+\vec{p}_{j}
\end{array}\right) \quad \mathrm{E} 0:\left(\begin{array}{c}
E_{i j}=E_{i}+E_{j} \\
\vec{p}_{i j}=E_{i j} \frac{\vec{p}_{i}+\vec{p}_{j}}{\left|\vec{p}_{i}+\vec{p}_{j}\right|}
\end{array}\right) \quad \mathrm{P}: \quad\left(\begin{array}{c}
E_{i j}=\left|\vec{p}_{i j}\right| \\
\vec{p}_{i j}=\left(\vec{p}_{i}+\vec{p}_{j}\right)
\end{array}\right) \text {. }
$$

The E-scheme is Lorentz invariant and strictly conserves energy and momentum. The E0-scheme conserves only energy, the P-scheme only momentum. Both schemes yield massless 4-vectors, which turns out to result in smaller theoretical uncertainties when comparing experimental data to the prediction from perturbative QCD. With a given metric and recombination scheme jets are reconstructed either until the minimum distance is above a certain threshold $y_{\text {cut }}$ or until a predefined number of jets is reached. Precise determinations of the strong coupling constant are obtained from the 3 -jet rate $R_{3}$ or the mean number of jets $\left\langle n_{\text {jet }}\right\rangle$ [61] measured for a certain value $y_{c u t}$, or from the distribution of the event-shape variable $y_{3}$, which, having clustered a given event until only 3 -jets remain, is the resolution parameter $y_{i j}$ where the event makes the transition from a 3 -jet to a 2 -jet configuration. It thus is also referred to as the "differential 2 -jet rate":

$$
y_{3}=\min \left(y_{12}, y_{23}, y_{31}\right) \quad \Rightarrow \quad \frac{1}{\sigma} \frac{d \sigma}{d y_{3}}=-\frac{d R_{2}}{d y_{\text {cut }}}
$$


In cases where variables depend on the choice of metric and recombination scheme this will be made explicit by quoting them with an argument (metric,scheme) which specifies the two. The quantity $y_{3}(\mathrm{D}, \mathrm{E})$ for example refers to $y_{3}$ as determined with the Durham-metric and the E-scheme.

The concept of jet-clustering can also be applied to the "classical" event-shape variables in order to reduce their sensitivity to hadronization effects. The ALEPH-collaboration [62] has done an analysis of pre-clustered Thrust, Oblateness, C-parameter and energy-energy-correlation, CT, $\mathrm{CO}, \mathrm{CC}$ and CEEC, where prior to evaluating the event-shape variables the tracks in the event were clustered according to the JADE-E0 scheme with resolution parameters $y_{\text {cut }}=0.03$ for $\mathrm{CT}, \mathrm{CO}$, $C C$ and $y_{c u t}=0.02$ for CEEC.

\subsubsection{Theoretical Predictions}

According to general theorems $[63,64,65]$ the perturbative prediction for the cumulative crosssection of any event shape variable $x$, which vanishes in the limit of perfect 2 -jet topologies, can be expressed in the form

$$
R(L) \equiv \frac{\sigma(-\ln (x)>L)}{\sigma_{\text {tot }}}=C\left(\alpha_{s}\right) \exp \left(G\left(\alpha_{s}, L\right)\right)+D\left(\alpha_{s}, L\right)
$$

with $C\left(\alpha_{s}=0\right)=1$ and $D(L \rightarrow \infty)=0$. The argument of the exponential function in general has the structure

$$
G\left(\alpha_{s}, L\right)=\sum_{n=1}^{\infty} \sum_{m=1}^{2 n} G_{n m} \alpha_{s}{ }^{n} L^{m} .
$$

The terms in this sum are classified as leading-logarithms for $m>n$, next-to-leading for $m=n$ and sub-leading for $m<n$. If the perturbative prediction exponentiates, one has $G_{n m}=0$ for $m>n+1$. In this case the perturbative prediction for $\ln R$ contains the following terms:

\begin{tabular}{|cc|cccc|}
\hline LL & NLL & \multicolumn{4}{|c|}{ Subleading Terms } \\
\hline$\alpha_{s} L^{2}$ & $\alpha_{s} L$ & $\alpha_{s}$ & $\alpha_{s} \mathrm{O}\left(\frac{1}{L}\right)$ & & \\
$\alpha_{s}{ }^{2} L^{3}$ & $\alpha_{s}{ }^{2} L^{2}$ & $\alpha_{s}{ }^{2} L$ & $\alpha_{s}{ }^{2}$ & $\alpha_{s}{ }^{2} \mathrm{O}\left(\frac{1}{L}\right)$ & \\
\hline$\alpha_{s}{ }^{3} L^{4}$ & $\alpha_{s}{ }^{3} L^{3}$ & $\alpha_{s}{ }^{3} L^{2}$ & $\alpha_{s}{ }^{3} L$ & $\alpha_{s}{ }^{3}$ & $\alpha_{s}{ }^{3} \mathrm{O}\left(\frac{1}{L}\right)$ \\
$\alpha_{s}{ }^{4} L^{5}$ & $\alpha_{s}{ }^{4} L^{4}$ & $\alpha_{s}{ }^{4} L^{3}$ & $\cdots$ & & \\
$\vdots$ & $\vdots$ & $\vdots$ & $\ddots$ & & \\
\hline
\end{tabular}

The first two columns are the leading-log and next-to-leading-log terms which for some event shape variables have been resummed into analytic functions $L G_{L L}\left(\alpha_{s} L\right)$ and $G_{N L L}\left(\alpha_{s} L\right)$. The first two rows constitute the theoretical prediction in second order perturbation theory. Based on numerical integration [67] of the ERT-matrix elements [54] the corresponding expressions are known for all event shape variables.

Having both the second order and the leading- plus next-to-leading-log resummed predictions, one can construct an improved theoretical prediction by combining the two. The combination is exact to $\mathrm{O}\left(\alpha_{s}^{2}\right)$ over the whole phase-space and in the vicinity of the 2 -jet region $(x \rightarrow 0)$ contains the dominant terms to all orders. 
There is a certain freedom in performing the matching of the theoretical predictions [66] which can be employed to probe the sensitivity of an $\alpha_{s}$ measurement to the unknown higher order corrections. Typical examples are the so called $\ln R$-matching and the $R$-matching schemes. For the $\ln R$-scheme the resummed prediction for $\ln R$ as displayed above is combined with the second order prediction. All terms in the lower right field of the above scheme are assumed to be zero. Exponentiating the resulting expression for $\ln R$, however, does create those kinds of terms in the prediction for $R$. Alternatively one can perform the matching for $R$. To $\mathrm{O}\left(\alpha_{s}^{2}\right)$ and in leadingand next-to-leading-log accuracy this gives exactly the same terms as the $\ln R$-matching, but now all sub-leading terms of higher than second order in $\alpha_{s}$ are zero. The two ways of matching thus differ in the unknown higher order terms.

Another common way to assess theoretical uncertainties is the variation of the renormalization scale. Technically this is done by using the solution of the $\beta$-function

$$
\alpha_{s}(Q)=\alpha_{s}(\mu)+\lambda \alpha_{s}^{2}(\mu)+\mathcal{O}\left(\alpha_{s}^{3}(\mu)\right) \quad \text { where } \quad \lambda=\frac{\beta_{0}}{4 \pi} \ln \frac{\mu^{2}}{Q^{2}}
$$

to substitute $\alpha_{s}(Q)$ by $\alpha_{s}(\mu)$ and then to reevaluate the modified theoretical prediction to the same formal precision as the original one. For the fixed second order QCD prediction this means

$$
\alpha_{s}(Q) A+\alpha_{s}^{2}(Q) B \rightarrow \alpha_{s}(\mu) A+\alpha_{s}^{2}(\mu)(B+\lambda A),
$$

where the functions $A$ and $B$ contain all kinematic terms proportional to $\alpha_{s}$ and $\alpha_{s}^{2}$, respectively. The resummed predictions transform according to

$$
\begin{array}{cccc}
L G_{L L}(v)+G_{N L L}(v) & \rightarrow & L G_{L L}(v)+G_{N L L}(v)+\lambda v^{2} \frac{\partial}{\partial v} G_{L L}(v) \\
v=\alpha_{s}(Q) L & \rightarrow & v=\alpha_{s}(\mu) L .
\end{array}
$$

Using the transformed prediction in an analysis of experimental data yields a measurement of $\alpha_{s}(\mu)$ instead of $\alpha_{s}(Q)$. Evolved back to the original scale $Q$, the resulting value for $\alpha_{s}(Q)$ in general will be different from the result of a measurement done directly at the scale $Q$. Formally this scale-dependence comes about because in the substitution step a truncated solution of the $\beta$-function is used, while the re-evolution uses a more complete expression. The whole procedure is mathematically equivalent to staying at the original scale $Q$ and introducing higher order terms of the type $\alpha_{s}^{3}(Q) \ln \left(\mu^{2} / Q^{2}\right)$. The scale dependence thus is of the order of the first uncalculated higher order corrections. For a complete perturbative prediction there would be no renormalization scale dependence.

Other ways to estimate the theoretical uncertainties of a perturbative prediction exist. Examples can be found in $[68,69,70]$. As a safeguard against accidental cancellations usually several methods are combined in order to assess the error due to unknown higher order perturbative effects.

Another class of theoretical uncertainties, which lies outside the domain of perturbative QCD, is due to the hadronization process. In some special cases the non-perturbative contributions are formally understood and can even be determined experimentally. Examples are deep-inelastic scattering experiments or the determination of $\alpha_{s}$ from hadronic $\tau$-decays. For $\alpha_{s}$-measurements based on event-shape variables the situation is less fortunate. Here the estimates of size and uncertainty of non-perturbative effects so far rely on Monte-Carlo models. 


\subsubsection{Experimental Results}

A compilation of measurements of $\alpha_{s}\left(M_{Z}\right)$ from single event shape variables is given in the appendix, sorted according to the individual experiments. Measurements are available based on the purely second order QCD prediction, the purely leading- plus next-to-leading-log resummed predictions and the combined ones. An example how the experimental data compare to the theoretical prediction, together with the size of the hadronization corrections is shown in fig. 11 for the Thrust distribution. The individual results for $\alpha_{s}\left(M_{Z}\right)$ using purely second order or resummed QCD predictions are displayed in fig.12 and fig.13, respectively. The errors are dominated by the theoretical uncertainties. The higher accuracy of the resummed predictions reflects in the smaller errors of the corresponding $\alpha_{s}$-measurements. In addition to the single measurements the LEP experiments also published combined results, based on whole sets of event shape variables, where the correlations between all contributing variables were taken properly into account. The individual averages are listed in table 2 and displayed together with a global average based on those numbers in fig. 14.

Due to the common theoretical background even different experiments are not independent. In order to combine the single experiment averages into one global average for $\alpha_{s}\left(M_{Z}\right)$, correlations between the contributing experiments have to be taken into account. A consistent way to achieve this even when the exact correlation pattern is unknown is presented in ref. [83]. Using the procedure described there and combining the single experiment averages into one "correlated average" yields $\alpha_{s}\left(M_{Z}\right)=0.1215 \pm 0.0059$, which is rounded to

$$
\left.\alpha_{s}\left(M_{Z}\right)\right|_{\text {event shapes }}=0.122 \pm 0.006 .
$$

All individual measurements within their error estimates are consistent with this global average. The error of the average is almost entirely theoretical and essentially determined by the more precise resummed results. As the theoretical error is the limitig factor in $\alpha_{s}$-determinations from event-shape variables, further improvements can only be expected from an improved theoretical prediction to $\mathrm{O}\left(\alpha_{s}^{3}\right)$.

\section{3 $\alpha_{s}$ from Scaling Violations}

The $Q^{2}$-dependence of structure functions observed in deep-inelastic scattering (DIS) experiments is a well established way to measure the strong coupling constant. In a completely analogous way it can be extracted from scaling violations in fragmentation functions which determine the inclusive momentum distribution in hadronic final states observed in $e^{+} e^{-}$-annihilation processes. The theoretical framework is described in detail in references [84, 85, 86, 87], experimental data sufficiently far away from all flavour thresholds are available between $\sqrt{s}=22 \mathrm{GeV}$ and $\sqrt{s}=91.2 \mathrm{GeV}$.

Determinations of $\alpha_{s}\left(M_{Z}\right)$ from scaling violations have to disentangle the logarithmic variations $\sim \ln (s)$ predicted by perturbative QCD from non-perturbative power-law corrections and trivial effects due to the change of the primary flavour composition when going from photon mediated lower energy processes towards the $Z$-resonance. An important difference to deep-inelastic scattering is the fact, that the non-perturbative effects for the time-like domain probed in $e^{+} e^{-}$annihilation are expected to decay only proportional to $1 / \sqrt{s}$, i.e. much slower than the $1 / Q^{2}$ 


\begin{tabular}{|c|c|c|c|c|c|}
\hline \multicolumn{2}{|c|}{ Experiment } & $\alpha_{s}\left(M_{Z}\right)$ & Total Error & Exp. Error & Theory \\
\hline ALEPH & {$[62]^{*}$} & 0.117 & $\pm_{0.010}^{0.008}$ & & $\mathrm{O}\left(\alpha_{s}^{2}\right)$ \\
\hline DELPHI & {$[71]^{*}$} & 0.113 & \pm 0.007 & $\pm \mathbf{0 . 0 0 2}$ & $\mathrm{O}\left(\alpha_{s}^{2}\right)$ \\
\hline L3 & {$[72]^{*}$} & 0.118 & \pm 0.010 & $\pm \mathbf{0 . 0 0 4}$ & $\mathrm{O}\left(\alpha_{s}^{2}\right)$ \\
\hline OPAL & [73] & 0.122 & \pm 0.007 & & $\mathrm{O}\left(\alpha_{s}^{2}\right)$ \\
\hline OPAL & {$[74]^{*}$} & 0.122 & $\pm_{0.005}^{0.006}$ & & $\mathrm{O}\left(\alpha_{s}^{2}\right)$ \\
\hline OPAL & [75] & 0.118 & \pm 0.008 & & $\mathrm{O}\left(\alpha_{s}^{2}\right)$ \\
\hline MARK II & {$[76]^{*}$} & 0.123 & \pm 0.010 & \pm 0.005 & $\mathrm{O}\left(\alpha_{s}^{2}\right)$ \\
\hline SLD & {$[77]^{*}$} & 0.118 & \pm 0.011 & $\pm \mathbf{0 . 0 0 4}$ & $\mathrm{O}\left(\alpha_{s}^{2}\right)$ \\
\hline ALEPH & {$[78]^{*}$} & 0.125 & \pm 0.005 & \pm 0.002 & $\mathrm{O}\left(\alpha_{s}^{2}\right)+\mathrm{LL}+\mathrm{NLL}$ \\
\hline ALEPH & [79] & 0.125 & \pm 0.005 & \pm 0.002 & $\mathrm{O}\left(\alpha_{s}^{2}\right)+\mathrm{LL}+\mathrm{NLL}$ \\
\hline DELPHI & [80] & 0.118 & \pm 0.007 & $\pm \mathbf{0 . 0 0 2}$ & $\mathrm{LL}+\mathrm{NLL}$ \\
\hline DELPHI & {$[80]^{*}$} & 0.123 & \pm 0.006 & $\pm \mathbf{0 . 0 0 2}$ & $\mathrm{O}\left(\alpha_{s}^{2}\right)+\mathrm{LL}+\mathrm{NLL}$ \\
\hline L3 & {$[81]^{*}$} & 0.124 & \pm 0.009 & \pm 0.003 & $\mathrm{O}\left(\alpha_{s}^{2}\right)+\mathrm{LL}+\mathrm{NLL}$ \\
\hline L3 & [82] & 0.125 & \pm 0.009 & \pm 0.003 & $\mathrm{O}\left(\alpha_{s}^{2}\right)+\mathrm{LL}+\mathrm{NLL}$ \\
\hline OPAL & {$[73]^{*}$} & 0.120 & \pm 0.006 & & $\mathrm{O}\left(\alpha_{s}^{2}\right)+\mathrm{LL}+\mathrm{NLL}$ \\
\hline SLD & {$[77]^{*}$} & 0.126 & \pm 0.007 & $\pm \mathbf{0 . 0 0 4}$ & $\mathrm{O}\left(\alpha_{s}^{2}\right)+\mathrm{LL}+\mathrm{NLL}$ \\
\hline
\end{tabular}

Table 2: Compilation of combined $\alpha_{s}$-measurements published by the LEP and SLC collaborations. The starred results are displayed in fig. 14 and also used in the determination of the global average for $\alpha_{s}\left(M_{Z}\right)$ from event-shape variables.

behaviour one has in DIS. As a consequence the dynamic range available to disentangle the effects of perturbative and non-perturbative QCD when comparing measurements between $\sqrt{s}=22 \mathrm{GeV}$ and $\sqrt{s}=91.2 \mathrm{GeV}$ is much smaller than the one available for the DIS-measurements, which cover the range between $Q^{2}=0.5 \mathrm{GeV}^{2}$ and $Q^{2}=260 \mathrm{GeV}^{2}[88]$.

The DELPHI-collaboration [89] first did this type of analysis at LEP, where the theoretical prediction was determined by the LUND matrix-element model with the cutoff of the perturbative phase held at a fixed mass. Using a complete Monte-Carlo model which combines fixed second order perturbative QCD with non-perturbative effects leaves only a small number of free parameters in addition to $\alpha_{s}\left(M_{Z}\right)$. From this a precise measurement of the strong coupling constant $\alpha_{s}\left(M_{Z}\right)=0.118 \pm 0.005$ was obtained, in good agreement with other determinations. A measurement based on the exact NLO theoretical framework without using information from a Monte-Carlo model was later presented by the ALEPH-Collaboration [90]. Here not only the strong coupling constant, but also parametrizations for the fragmentation functions of all quark flavours and the gluon together with the energy dependence of the non-perturbative effects were extracted from the data. The result of this model-independent analysis is $\alpha_{s}\left(M_{Z}\right)=0.127 \pm 0.011$. The larger error is the price that is paid for a reduced model dependence. A comparison between the experimental data and the QCD prediction is shown in fig. 15 . 


\section{$4.4 \quad \alpha_{s}$ from $R_{\tau}$}

Another fully inclusive measurement of the strong coupling constant can be obtained from the analysis of hadronic $\tau$-decays. The quantity $R_{\tau}$

$$
R_{\tau}=\frac{\Gamma\left(\tau^{-} \rightarrow \text { hadrons }\right)}{\Gamma\left(\tau^{-} \rightarrow \nu_{\tau} \bar{\nu}_{e} e^{-}\right)}=3 S_{E W}\left(1+\delta_{E W}+\delta_{\mathrm{QCD}}+\delta_{m}+\delta_{n p}\right)
$$

contains information about the strong coupling constant in the same way as does $R_{Z}$. In the above expression $S_{E W}=1.0194$ and $\delta_{E W}=0.0010$ are the purely electroweak corrections to $R_{\tau}$, $\delta_{\mathrm{QCD}}$ the perturbative QCD correction for massless quarks, $\delta_{m}$ the corrections due to the finite quark masses and $\delta_{n p}$ the non-perturbative correction in the Shifman-Vainshtein-Zhakharov (SVZ) approach [91]. The theoretical prediction is described in detail in [92, 93, 94, 95]. Here only a short summary can be given.

The perturbative correction $\delta_{\mathrm{QCD}}$ to $R_{\tau}$ is obtained by integrating the $\mathrm{O}\left(\alpha_{s}^{3}\right)$ correction to $R_{\gamma}$, the ratio of hadronic to the leptonic cross section for $e^{+} e^{-}$-annihilation into virtual photons, over the mass spectrum of the hadronic system produced in $\tau$-decays. Technically this is done via a contour integral which sums up certain terms of the perturbative expansion to all orders [93] and leads to a rapidly converging series. The result as function of $\alpha_{s}\left(M_{\tau}\right)$ cannot be expressed in terms of elementary functions in closed form. An empirical parametrization is

$$
\delta_{\mathrm{QCD}}=0.0368-0.1706 \alpha_{s}+2.8013 \alpha_{s}{ }^{2}-2.8520 \alpha_{s}{ }^{3} \pm \alpha_{s}{ }^{3}\left(0.1939-2.7388 \alpha_{s}+4.4160 \alpha_{s}{ }^{2}\right) .
$$

In the range $0.24<\alpha_{s}\left(M_{\tau}\right)<0.44$ the exact next-to-next-to-leading order result is reproduced with a relative precision better than $0.02 \%$. The only relevant source of theoretical uncertainty in $\delta_{\mathrm{QCD}}$ comes from the unknown $\mathcal{O}\left(\alpha_{s}^{4}\right)$ correction to $R_{\gamma}$. The estimate given above was obtained by varying the coefficient of a fourth order term by \pm twice the size expected from a geometric progression. The quark mass dependent corrections can be parametrized by

$$
\delta_{m}=-0.0041-0.0136 \alpha_{s}-0.0439 \alpha_{s}^{2} \pm\left(0.0004+0.0028 \alpha_{s}+0.0096 \alpha_{s}^{2}\right) .
$$

Assuming the validity of the SVZ-approach implies that all power-law corrections proportional to $1 / M_{\tau}{ }^{2}$ are contained in $\delta_{m}$. The remaining non-perturbative terms $\delta_{n p}$ can then be organized as

$$
\delta_{n p}=\frac{11}{4} \alpha_{s}{ }^{2} \frac{\left\langle\frac{\alpha_{s}}{\pi} G G\right\rangle}{m_{\tau}^{4}}-12 \pi^{2} \frac{O(6)}{m_{\tau}^{6}}-8 \pi^{2} \frac{O(8)}{m_{\tau}^{8}}+\mathcal{O}\left(1 / m_{\tau}^{10}\right),
$$

where $\left\langle\frac{\alpha_{s}}{\pi} G G\right\rangle$ is the so-called Gluon-condensate and $O(6)$ and $O(8)$ vacuum expectation values of dimension- 6 and 8 operators. Numerical values from phenomenological fits to different sets of data are quoted in $[92,94]$ as $\left\langle\frac{\alpha_{s}}{\pi} G G\right\rangle=0.02 \pm 0.01 \mathrm{GeV}^{4}$ and $O(6)=0.002 \pm 0.001 \mathrm{GeV}^{6}$. The $O(8)$-term is neglected there. As described in [94] one can determine these condensates also from moments of the invariant mass distribution of hadronic $\tau$-decays, thus extracting simultaneously the strong coupling constant and the non-perturbative corrections. Such an analysis was performed by the ALEPH collaboration [68], where the condensate terms were measured in Cabibbo-allowed transitions. The (highly correlated) results are $\left\langle\frac{\alpha_{s}}{\pi} G G\right\rangle=0.02 \pm 0.02 \mathrm{GeV}^{4}$, $O(6)=-0.003 \pm 0.002 \mathrm{GeV}^{6}$ and $O(8)=0.003 \pm 0.003 \mathrm{GeV}^{8}$. Preliminary new results are given in $[96,97]$. 
Experimental measurements of $R_{\tau}$ are obtained from the leptonic branching ratios. Assuming the validity of the completeness relation for the $\tau$-branching ratios into hadrons, electrons and muons, $B_{\text {hadr }}+B_{e}+B_{\mu}=1$, the ratio $R_{\tau}$ can be expressed as

$$
R_{\tau}=\frac{1-B_{e}-B_{\mu}}{B_{e}}=\frac{1}{B_{e}}-1-f_{\mu}=f_{\mu}\left(\frac{1}{B_{\mu}}-1\right)-1 \quad \text { with } \quad f_{\mu} \equiv \frac{B_{\mu}}{B_{e}} .
$$

The factor $f_{\mu}=0.9728$ takes into account that the muon decay is slightly suppressed due to the larger mass of the muon. Using the calculated value for $f_{\mu}$ as input, two independent determinations of $R_{\tau}$ are obtained from $B_{e}$ and $B_{\mu}$. Another measurement is extracted by comparing the $\tau$-mass and lifetime to the muon mass and lifetime. Assuming lepton universality the electro-weak standard model predicts

$$
\frac{\Gamma_{e}(\mu)}{\Gamma_{e}(\tau)}=\frac{\Gamma_{t o t}(\mu)}{B_{e} \Gamma_{t o t}(\tau)}=\frac{m_{\mu}^{5}}{m_{\tau}^{5}} \quad \Rightarrow \quad B_{e}=\frac{\tau_{\tau}}{\tau_{\mu}}\left(\frac{m_{\tau}}{m_{\mu}}\right)^{5}
$$

Using the current world averages $m_{\tau}=1777.0 \pm 0.3 \mathrm{MeV} / c^{2}[98], \tau_{\tau}=290.8 \pm 1.5 \mathrm{fs}$ [98], $B_{e}=0.1766 \pm 0.0011[98]$ and $B_{\mu}=0.1742 \pm 0.0017[99,100]$ gives the combined result $R_{\tau}=3.655 \pm 0.022$. From this the value of $\alpha_{s}\left(M_{\tau}\right)$ is inferred as

$$
\alpha_{s}\left(M_{\tau}\right)=0.373 \pm 0.010(\exp ) \pm_{0.031}^{0.041}(\text { theo })
$$

with the condensate terms taken from $[92,94]$.

In order to compare this measurement to $\alpha_{s}$-determinations done at the scale of the $Z$-mass, $\alpha_{s}\left(M_{\tau}\right)$ has to be evolved up to $M_{Z}$. Runge-Kutta integration of the NNLO beta-function yields

$$
\alpha_{s}\left(M_{Z}\right)=0.125 \pm 0.001(\exp ) \pm 0.004(\text { theo }) \pm 0.001(\text { evol })=0.125 \pm 0.004
$$

The value for $\alpha_{s}\left(M_{\tau}\right)$ was determined for $n_{f}=3$ active flavours. Following [46] the evolution to the 5 -flavour $\alpha_{s}\left(M_{Z}\right)$ was done by first going back to the charm threshold $\mu_{t h}^{c}=1.4 \pm 0.2 \mathrm{GeV}$ with the beta-function for three flavours, then going from $\mu_{t h}^{c}$ to the bottom threshold $\mu_{t h}^{b}=4.7 \pm 0.5 \mathrm{GeV}$ with the 4-flavour evolution equation, and finally from $\mu_{t h}^{b}$ to $M_{Z}$ with the 5 -flavour beta-function. The result is in good agreement with the measurement from $R_{Z}$ and event shape variables, thus supporting the SVZ-ansatz. The evolution error of 0.001 contains the uncertainties from the position of the flavour thresholds, which were taken to be fully correlated, plus the effect of missing higher order terms in the beta-function. The latter uncertainty again was estimated by adding a next higher order term with a coefficient of \pm twice the size expected from a geometric progression.

\subsection{The Running of $\alpha_{s}$}

One of the fundamental tests of QCD is to verify that $\alpha_{s}$-measurements done at different renormalization scales are related as decribed by the $\beta$-function eq.(2). LEP alone provides two points, $\alpha_{s}\left(M_{\tau}\right)$ and $\alpha_{s}\left(M_{Z}\right)$. For $\alpha_{s}\left(M_{Z}\right)$ two independent measurements are available, one from $R_{Z}$ and one from global event shapes. In order to test that QCD really is the universal theory describing strong interaction processes further information has to be included. As detailed 
compilations can be found e.g. in $[101,102]$, here only a short summary of the available material will be presented.

Measurements of the strong coupling constant exist from lepton-nucleon scattering experiments with neutrino, electron and muon beams. Using charged leptons, values for $\Lambda \frac{(4)}{\mathrm{MS}}$ are published by the BCDMS [103] and EMC [104]/NMC [105] collaboration and in a combined analysis of SLAC and BCDMS data [88]. Results from neutrino beams are given by the CHARM [106], CDHSW [107] and the CCFR [108] collaboration. All numbers in good agreement. The average quoted below uses the re-analyzed CDHSW-result as presented and discussed in detail in reference [109]. The weighted average considering only the experimental errors is $\Lambda_{\overline{\mathrm{MS}}}^{(4)}=245 \pm 26 \mathrm{MeV}$, with a $\chi^{2} / \mathrm{df}=2.6 / 6$. Assuming that the theoretical uncertainties determined in [88] apply throughout, one obtains at the typical scale quoted for DIS experiments $\alpha_{s}(7.1 \mathrm{GeV})=0.177 \pm 0.005 \pm 0.010$.

A measurement of $\alpha_{s}$ from the analysis of heavy quarkonia decays is given in [70]. Expressed at a reference scale $Q=10 \mathrm{GeV}$ the result is $\alpha_{s}(10 \mathrm{GeV})=0.167 \pm_{0.011}^{0.015}$.

Another measurement is based on $R_{\gamma}$ which is sensitive to $\alpha_{s}$ and the weak mixing angle $\Theta_{w}$. Results are available based on data with centre-of-mass energies between $7 \mathrm{GeV}$ and $57 \mathrm{GeV}[110]$, and on data with centre-of-mass energy $<52 \mathrm{GeV}$ [111]. Fixing $\sin ^{2} \Theta_{w}$ to the LEP average [112], the mean value of both results expressed at a common scale is $\alpha_{s}(31.6 \mathrm{GeV})=0.142 \pm 0.015$. As both measurements are based on essentially the same data the combined error is taken to be the smaller of the two individual errors. The published analyses are using the erroneous coefficient $k_{3}=64.8[113]$ instead of $k_{3}=-12.8[114,115]$ for the $\mathrm{O}\left(\alpha_{s}^{3}\right)$ correction to $R_{\gamma}$. Correcting for this, finally yields $\alpha_{s}(31.6 \mathrm{GeV})=0.163 \pm 0.022$.

Non-LEP/SLC measurements of the strong coupling constant from event shape variables exist from CESR, PEP/PETRA and TRISTAN for energies around $10 \mathrm{GeV}, 29 \mathrm{GeV} / 34 \mathrm{GeV}$ and $58 \mathrm{GeV}$, respectively. The recent CESR result based on jet production rates is $\alpha_{s}(10.53 \mathrm{GeV})=$ $0.165 \pm 0.018$ (CLEO, [116]). The PEP/PETRA results are summarized by $\alpha_{s}(34 \mathrm{GeV})=$ $0.14 \pm 0.02[117,118,119]$. Measurements quoted from TRISTAN are based on fitting the NLLJET Monte-Carlo to the data, where the theoretical content of the Monte-Carlo corresponds to the next-to-leading-log improved second order QCD prediction. For $Q=58 \mathrm{GeV}$ the experiments found $\alpha_{s}(Q)=0.129 \pm 0.004 \pm_{0.005}^{0.004}($ VENUS, $[120]), \alpha_{s}(Q)=0.134 \pm_{0.005}^{0.006}(\mathrm{AMY},[121])$ and $\alpha_{s}(Q)=0.125 \pm 0.009$ (TOPAZ, [122]). The TOPAZ collaboration also determined a value for $\alpha_{s}$ using the analytical predictions instead of the Monte-Carlo model as $\alpha_{s}(58 \mathrm{GeV})=0.132 \pm 0.008$. The correlated average of those results yields $\alpha_{s}(58 \mathrm{GeV})=0.131 \pm \mathbf{0 . 0 0 6}$.

With progress in lattice gauge calculations also improved determinations of the strong coupling constant from analysing level splittings in the charmonium and upsilon system became available. While the first results [123] were still significantly below the LEP numbers, later calculations with an improved conversion of the coupling constant evaluated on the lattice towards the $\overline{\mathrm{MS}}$ coupling constant of perturbative QCD gave larger values [124, 125]. The currently quoted result from lattice calculations is $\alpha_{s}(5 \mathrm{GeV})=0.203 \pm 0.010$, corresponding to $\alpha_{s}\left(M_{Z}\right)=$ $0.115 \pm 0.003[126,127]$.

Other measurements of $\alpha_{s}$ come from collider experiments. An analysis of $b \bar{b}+$ jets production [128] gives $\alpha_{s}(20 \mathrm{GeV})=0.138 \pm_{0.019}^{0.028}$, a study of jet-production in W-decays [129] yields $\alpha_{s}(80.6 \mathrm{GeV})=0.123 \pm 0.025$. A further determination of $\alpha_{s}$ at low $Q^{2}$ comes from a comparison of direct photon production in proton-proton and proton-antiproton collisions [130]. 
At the typical scale $Q=P_{T}=4 \mathrm{GeV}$ the value found for $\Lambda_{\overline{\mathrm{MS}}}^{(4)}=235 \pm 106 \pm_{9}^{146} \mathrm{MeV}$ corresponds to $\alpha_{s}(4 \mathrm{GeV})=0.206 \pm_{0.032}^{0.045}$.

\begin{tabular}{|c|c|c|c|}
\hline Measurement & $\mathrm{Q} / \mathrm{GeV}$ & $\alpha_{s}(Q)$ & $\alpha_{s}\left(M_{Z}\right)$ \\
\hline$R_{\tau}$ & 1.777 & $0.373 \pm \begin{array}{l}0.042 \\
0.033\end{array}$ & $0.125 \pm 0.004$ \\
\hline$p \bar{p}, p p \rightarrow \gamma+\mathrm{X}$ & 4.0 & $0.206 \pm \begin{array}{l}0.045 \\
0.032\end{array}$ & $0.112 \pm \begin{array}{l}0.012 \\
0.010\end{array}$ \\
\hline Lattice Gauge Theory & 5.0 & $0.203 \pm 0.010$ & $0.115 \pm 0.003$ \\
\hline DIS & 7.1 & $0.177 \pm 0.011$ & $0.113 \pm 0.005$ \\
\hline $\mathrm{J} / \Psi, \Upsilon$ & 10.0 & $0.167 \pm \begin{array}{l}0.015 \\
0.011\end{array}$ & $0.113 \pm \begin{array}{l}0.007 \\
0.005\end{array}$ \\
\hline$e^{+} e^{-} \rightarrow$ hadrons: event shapes & 10.53 & $0.165 \pm 0.018$ & $0.113 \pm 0.006$ \\
\hline$p \bar{p} \rightarrow b \bar{b}+\mathrm{jets}$ & 20.0 & $0.138 \pm \begin{array}{l}0.028 \\
0.019\end{array}$ & $0.109 \pm \begin{array}{l}0.016 \\
0.012\end{array}$ \\
\hline$R_{\gamma}$ & 31.6 & $0.163 \pm 0.022$ & $0.133 \pm 0.015$ \\
\hline$e^{+} e^{-} \rightarrow$ hadrons: event shapes & 34.0 & $0.140 \pm 0.020$ & $0.119 \pm 0.015$ \\
\hline$e^{+} e^{-} \rightarrow$ hadrons: event shapes & 58.0 & $0.131 \pm 0.006$ & $0.122 \pm 0.005$ \\
\hline$p \bar{p} \rightarrow \mathrm{W}+\mathrm{jets}$ & 80.6 & $0.123 \pm 0.025$ & $0.121 \pm 0.024$ \\
\hline$R_{Z}$ & 91.2 & $0.126 \pm 0.007$ & $0.126 \pm 0.007$ \\
\hline $\mathrm{Z} \rightarrow$ hadrons: event shapes & 91.2 & $0.122 \pm 0.006$ & $0.122 \pm 0.006$ \\
\hline
\end{tabular}

Table 3: Summary of $\alpha_{s}$-measurements at different energies. The result from lattice gauge theories is based on measurements of the level-splitting in the $\Upsilon$-system.

Table 3 gives an overview over the energy dependence of $\alpha_{s}$. The data are displayed in fig.16. Evolved up to the $Z$-mass all measurements are compatible with one common value. The correlated average according to $[83]$ is $\alpha_{s}\left(M_{Z}\right)=0.1183 \pm 0.0025$. A more conservative average is obtained if the two most precise measurements, $\alpha_{s}$ from lattice gauge theories and the measurement of $\alpha_{s}$ from $R_{\tau}$ are ignored. This more cautious approach is motivated by the fact, that the former measurement has been less stable in the past while the latter one is potentially controversial as the insensitivity to non-perturbative effects which follows from the assumption of the SVZ-approach may not be valid $[102,131,132]$. One then obtains the global average

$$
\alpha_{s}\left(M_{Z}\right)=0.1180 \pm 0.0045
$$

Some remarks are in order here. First, this global average contains a certain subjective element in the way the input data were selected. Here the point of view was taken, to use all types of measurements on equal footing and exploit the fact that the averaging procedure [83] to some extent can account for correlations in the data. An alternative would be to pick by hand single and - hopefully - almost uncorrelated measurements and average those. Trying this the central value for $\alpha_{s}\left(M_{Z}\right)$ essentially remains unchanged, but the error typically decreases by $30 \%$, i.e. the 
above result can be viewed as a conservative estimate for the precision to which $\alpha_{s}\left(M_{Z}\right)$ is known today.

It also has to be pointed out, that single determinations of $\alpha_{s}\left(M_{Z}\right)$ with a precision in the 0.002-range are becoming available from lattice-gauge calculations and the analysis of hadronic $\tau$ decays, where in addition to $R_{\tau}$ the non-perturbative terms in the framework of the SVZ-approach are extracted from the spectral moments of the hadronic mass spectrum [96]. If the high precision for either of the two will be confirmed, they will dominate the future knowledge about the strong coupling constant.

Finally it should be noted, that the measurements of the strong coupling constant apparently fall into two groups, although at the current level of uncertainty all results are consistent. No unambigous pattern emerges, but it seems that either the low-energy measurements or those measurements which are affected by bound state effects, like nucleon structure functions or Charmonium and Upsilon wave functions, tend towards lower values for $\alpha_{s}$ while the complementary set seems to prefer larger values. It will be interesting to see, whether this trend persists in the future.

\subsection{Tests of the Flavour Independence of $\alpha_{s}$}

According to QCD the strong coupling constant is the same for all quark flavours. The fact that the measurements of $\alpha_{s}$ from a multitude of reactions and energy scales appear to be consistent with a common value for $\alpha_{s}\left(M_{Z}\right)$ already supports this notion. More direct tests can be performed with dedicated measurements of the strong coupling constant based on data samples where the composition of the active quark flavours can be controlled experimentally. Any observation of a flavour dependence would be a strong indication for new physics, either by establishing a dependence between quark flavour and colour charge or by pointing towards new particles that couple to the strong interactions sector.

Allowing for a flavour dependence of the strong coupling constant, the second order QCD prediction for the differential cross section of a global event shape variable from hadronic $Z$-decays into a quark-antiquark pair of flavour $f$ can be written as

$$
\frac{d \sigma(f)}{d x}=A(x, f) \alpha_{s}(f)+B(x, f) \alpha_{s}^{2}(f)
$$

The functions $A(x, f)$ and $B(x, f)$ are kinematic functions where the flavour dependence enters through the different quark masses. For $b$-quarks those purely kinematic effects amount to a correction of about $5 \%$ in the 3 -jet cross section at a JADE-resolution parameter $y_{\text {cut }}^{J}=0.04$, i.e. for a precision test of the flavour independence of $\alpha_{s}$ they cannot be ignored. For the other quark flavours the mass effects are negligible. The quark mass dependence of $A(x, f)$ has been fully calculated $[133,134]$. For $B(x, f)$ only a partial evaluation of the mass effects exists $[135,136]$.

Experimentally various tagging techniques exist to select data samples with different flavour compositions. Requiring for instance a lepton with large $\mathrm{p}_{T}$ relative to the Thrust axis, a displaced secondary vertex or large impact parameters in an event yields a $b$-quark enriched sample. Antitagging on lifetime or simply requiring a leading particle in the event with a momentum of more than $70 \%$ of the beam momentum enriches light flavours $u d s$. Selecting $\mathrm{D}^{*} \mathrm{~s}$ or leading $\mathrm{K}^{0} \mathrm{~s}$ 


\begin{tabular}{|ll|ll|}
\hline \multicolumn{3}{|c|}{$\alpha_{s}(b) / \alpha_{s}(u d s c)$} \\
\hline $0.996 \pm_{0.023}^{0.026}(0.016)$ & ALEPH & {$[137]$} \\
$1.014 \pm_{0.027}^{0.037}$ & & ALEPH & {$[138]$} \\
$1.00 \pm 0.05(0.04)$ & DELPHI & {$[139]$} \\
$1.00 \pm 0.08(0.05)$ & L3 & {$[140]$} \\
$1.017 \pm 0.036(0.016)$ & OPAL & {$[141]$} \\
$0.992 \pm{ }_{0.017}^{0.015}(0.007)$ & OPAL & {$[142]$} \\
\hline $0.999 \pm 0.025$ & \multicolumn{2}{|l|}{ LEP Average } \\
\hline
\end{tabular}

Table 4: Measurements of $\alpha_{s}(b) / \alpha_{s}(u d s c)$ based on lepton-tag $[137,139,140,141]$ or lifetimetag $[138,142]$ in order to obtain a $b$-quark enriched sample. The average of the correlated individual results was calculated according to reference [83]. The errors are the total uncertainties. In parentheses the purely statistical componenent is given.

\begin{tabular}{|c|c|c|}
\hline Measurement & Result & Correlation Coefficients \\
\hline$\alpha_{s}(u d s) / \alpha_{s}($ incl. $)$ & $0.99 \pm 0.06(0.03)$ & 1. \\
\hline$\alpha_{s}(c) / \alpha_{s}($ incl. $)$ & $1.05 \pm 0.24(0.11)$ & -0.79 \\
\hline$\alpha_{s}(b) / \alpha_{s}($ incl. $)$ & $1.02 \pm 0.08(0.04)$ & $+0.26-0.51$ \\
\hline
\end{tabular}

Table 5: Tests of the flavour independence of $\alpha_{s}$ performed by the SLD-collaboration [143]. The various flavours are separated using impact parameter information. The errors are the total uncertainties. In parentheses the purely statistical component given.

produces a sample enriched in $c$ - or $s$-quarks, respectively. The actual flavour composition due to a specfic tag is usually estimated by Monte-Carlo simulations. When measuring ratios of the strong coupling constant for a tagged compared to the complementary quark flavours most of the otherwise dominant theoretical errors cancel.

The most obvious test, probing the mass dependence of $\alpha_{s}$, is a measurement of $\alpha_{s}(b) / \alpha_{s}(u d s c)$. Measurements done by the LEP-collaborations are collected in table 4. All experiments find a ratio compatible with unity. The correlated average [83] is $\alpha_{s}(b) / \alpha_{s}(u d s c)=0.999 \pm 0.025$, which constitutes the so far most stringent limit on a flavour dependence of $\alpha_{s}$. Apart from mass dependence one can study whether a flavour dependence of the strong coupling might be correlated with electric charge or weak isospin. No dependence on any of those quantum numbers was found [141].

Lifetime information allows to distinguish between $b, c$ and light flavours. A corresponding analysis was done by the SLD-collaboration [143]. The results as shown in table 5 are perfectly consistent with the assumption that the strong coupling constant is flavour independent. 


\begin{tabular}{|c|c|c|c|c|c|c|}
\hline Measurement & Result & \multicolumn{5}{|c|}{ Correlation Coefficients } \\
\hline$\alpha_{s}(u) / \alpha_{s}$ (incl.) & $0.951 \pm 0.209(0.103)$ & 1. & & & & \\
\hline$\alpha_{s}(d) / \alpha_{s}($ incl. $)$ & $0.933 \pm 0.195(0.087)$ & -0.531 & 1. & & & \\
\hline$\alpha_{s}(s) / \alpha_{s}($ incl. $)$ & $1.141 \pm 0.148(0.043)$ & -0.348 & -0.386 & 1. & & \\
\hline$\alpha_{s}(c) / \alpha_{s}($ incl. $)$ & $0.912 \pm 0.091(0.067)$ & -0.180 & -0.345 & -0.051 & 1. & \\
\hline$\alpha_{s}(b) / \alpha_{s}$ (incl. $)$ & $1.021 \pm 0.026(0.013)$ & -0.010 & -0.002 & -0.036 & -0.207 & 1. \\
\hline
\end{tabular}

Table 6: Measurements by the OPAL collaboration [141] of the strong coupling constant for all individual quark flavours. In addition to an untagged sample, data sets with different flavour composition were selected using high- $\mathrm{p}_{T}$ leptons $, \mathrm{D}^{*}, \mathrm{~K}^{0}$ and leading particle tags. From these the $\alpha_{s}$-values for the individual quark flavours were unfolded. The errors are the total uncertainties. In parentheses the purely statistical component is given. Note that the correlation coefficients are updated [144] with respect to the original reference [141].

Ultimately all results concerning the flavour independence of $\alpha_{s}$ can be expressed through the ratios $\alpha_{s}(i) / \alpha_{s}$ (incl.) with $i=u, d, s, c, b$. Such a measurement can be obtained from any set of five data samples with different flavour composition. In a first analysis of this kind [141] all ratios were found to be compatible with unity. The results together with the full correlation matrix are given in table 6 .

\section{Colour Factors of QCD}

Another important test of QCD is to verify that the dynamics is decribed by an unbroken SU(3) gauge symmetry. Although it is known that quarks come in three "colours", the relation between these internal degrees of freedom and the dynamics of strong interactions is not fixed a priori. Assuming that all three colours are charges of strong interactions suggests a simple Lie-group like $\mathrm{SU}(3), \mathrm{SO}(3)$ or an abelian $\mathrm{U}(1)_{3}$. Only the additional input that three quarks or a quarkantiquark pair can exist in a colour neutral state singles out $\mathrm{SU}(3)$. Accepting that quarks transform as $\mathrm{SU}(3)$ triplets it is still conceivable that not all internal degrees of freedom of contribute to the dynamics of QCD. In this case subgroups of SU(3) like $\mathrm{SU}(2), \mathrm{SO}(2)$ or $\mathrm{U}(1)$ become possible candidates for the gauge symmetry. Going one step further one can also imagine strong interactions to be described by a spontaneously broken SU(3) symmetry. The resulting massive gauge bosons would lead to a dynamical structure which would deviate from the SU(3) expectation. Deviations can also be caused by the existence of new physics which couples to the strong interactions sector. A popular example for the latter is the case of a light gluino, the supersymmetric partner of the gluon, which at $O\left(\alpha_{s}^{2}\right)$ contributes three additional fermionic degrees of freedom in $e^{+} e^{-}$-annihilation processes [69].

Experimentally the full gauge structure of QCD becomes accessible in $\mathrm{O}\left(\alpha_{s}^{2}\right)$. The types of diagrams contributing to the process $e^{+} e^{-} \rightarrow$ Hadrons at that order are shown in fig.17. In addition to the abelian double-bremsstrahlung diagrams $(\mathrm{a}, \mathrm{b})$ and the splitting of an intermediate 
gluon into a secondary quark-antiquark pair (d) there is also the process of a gluon splitting into secondary gluons (c), the defining characteristic of a non-abelian gauge theory.

The contribution of individual diagrams to the observable cross section is not gauge invariant. It is therefore not possible to experimentally isolate e.g. the triple-gluon contribution to the 4-jet cross section. A gauge invariant way to probe the structure of the underlying theory is to measure the Casimir operators or "Colour factors" $C_{F}, C_{A}$ and $T_{F}$. For a given representation of a gauge group describing the interaction, the colour factors are defined through the structure constants $f^{a b c}$ and the generators $T_{i j}^{a}$ :

$$
\sum_{a=1}^{N_{A}}\left(T^{a} T^{\dagger a}\right)_{i j}=\delta_{i j} C_{F} \quad, \quad \sum_{a, b=1}^{N_{A}} f^{a b c} f^{* a b d}=\delta^{c d} C_{A} \quad, \quad \sum_{i, j=1}^{N_{F}} T_{i j}^{a} T_{j i}^{\dagger b}=\delta^{a b} T_{F} .
$$

For QCD the generators are proportional to the Gell-Mann matrices $T_{i j}^{a}=\lambda_{i j}^{a} / 2$. The colour factor $C_{F}$ is the Casimir operator of the fermionic representation with dimension $N_{F}, C_{A}$ the one of the adjoint representation of the gluons with dimension $N_{A}$. Summing over all indices in the defining equations for $C_{F}$ and $T_{F}$ one finds $N_{F} C_{F}=N_{A} T_{F}$, i.e. a connection between the dimensionalities of the fermionic and the gluonic representation:

$$
\frac{T_{F}}{C_{F}}=\frac{N_{F}}{N_{A}}
$$

In an intuitive way the colour factors can be identified with the fundamental couplings of the theory as illustrated in fig.18. The factor $C_{F}$ determines the coupling strength of a gluon to a quark or antiquark, $C_{A}$ the strength of the gluon self-coupling and $T_{F}$ the probability for the splitting of a gluon into a quark-antiquark pair. In other words, $C_{F}$ and $C_{A}$ can be viewed as the square of the colour charge of a quark and a gluon, respectively. Absorbing a factor $C_{F}$ into the definition of the coupling constant one sees, that the gauge structure of the underlying theory can be parametrized by two ratios: $C_{A} / C_{F}$, the ratio of gluon-self coupling to the quark-gluon coupling, and $T_{F} / C_{F}=N_{F} / N_{A}$, the number of colours caried by the quarks divided by the number of gluons.

Experimentally colour factor information can be extracted from every process where the theoretical prediction is known at least to $\mathrm{O}\left(\alpha_{s}^{2}\right)$. Typical examples from $e^{+} e^{-}$-annihilation processes are the 2 -jet rate as function of the jet-resolution parameter $y_{\text {cut }}$ or the internal structure of 3 - and 4-jet events. The generic form of the differential cross section can be written as

$$
d^{n} \sigma=\left(\alpha_{s} C_{F}\right) A+\left(\alpha_{s} C_{F}\right)^{2} B\left(\frac{C_{A}}{C_{F}}, \frac{T_{F}}{C_{F}} n_{f}\right)+\mathrm{O}\left(\alpha_{s}^{3}\right)
$$

with $n=1$ for the 2 -jet rate, $n=2$ to describe the kinematics of 3 -jet events and $n=5$ to specify the internal structure of 4 -jet events. The functions $A$ and $B$ are universal kinematic functions which are the same for all types of unbroken gauge symmetries based on a simple Lie-group. The dependence on the gauge group enters through $B$, which is a function of the colour factor ratios. The contributions from the gluon-splitting process proportional to $T_{F} / C_{F}$ is always multiplied with the number of active fermion flavours $n_{f}$. The function $A$ is independent of the gauge structure. Measurements of colour factors at LEP exist based on 2-, 3- and 4-jet events. In the case of 4-jet events the coefficient $A$ is zero and the gauge structure determines the theoretical prediction at 
leading order. For 2- and 3-jet events it appears in the next-to-leading order corrections, which, despite higher statistics, renders a measurement more difficult.

Two approaches are used in the analysis of 4-jet events. The first one is an unbinned maximum likelihood fit of the colour factor ratios to the observed 5-dimensional differential cross section $[145,146,147]$. This guaranties that all available information is used in the measurement at the expense that the quality of the fit is difficult to asses. A more intuitive approach is based on using test-variables with particular sensitivity to the gauge structure of the theory. Candidate variables are discussed in $[148,149,150,151,152]$, comparisons with experimental data can be found in $[153,154,155,156,157]$. In all cases the results are found to be compatible with the QCD prediction, while an abelian toy-model, based on an $\mathrm{U}(1)_{3}$ gauge symmetry, could be excluded. Actual measurements of the colour factors based on test variables were done in $[158,159,160,161]$. The analysis by the DELPHI-collaboration for instance is based on the 2-dimensional distribution of the opening angle $\alpha_{34}$ between the two lowest energy jets and the modified Nachtmann-Reiter angle $\Theta_{\mathrm{NR}}^{*}$ [152], defined as the angle between the difference of the momentum vectors of the two highest energy jets and the two lowest energy jets. Both test variables are motivated by the properties of the diagrams where an intermediate gluon splits into a pair of secondary partons fig.17(c) and (d). The contribution of these gluon-splitting processes to the observed cross section is sensitive to $\alpha_{34}$. As the low energy jets are produced dominantly from higher order radiative corrections and small opening angles imply small invariant masses, one has a handle to control the contribution from the gluon propagator which also favours low mass systems. The other variable $\Theta_{\mathrm{NR}}^{*}$ probes the spin structure of the gluon splitting and thus is sensitive to the gauge structure of the theory. An angle around $\Theta_{\mathrm{NR}}^{*}=90^{\circ}$ is suppressed for a gluon splitting into a quark-antiquark pair while no suppression exists for a splitting into secondary gluons.

A compilation of the most recent LEP analyses, partially superseding previously shown results, is given in table 7. Colour-factor measurements based on 4 -jet events were presented by all four LEP experiments. The ALEPH collaboration [162] has also performed a measurement using information from 2- and 3-jet events. Although the uncertainties are large, the correlations are such that for $T_{F} / C_{F}>0$ a value $C_{A} / C_{F}=0$ is excluded with more than 4.7 standard deviations, giving clear evidence for the non-abelian nature of QCD. Combining these colour factor measurements one obtains $C_{A} / C_{F}=2.22 \pm 0.22$ and $T_{F} / C_{F}=0.33 \pm 0.12$. The partial average over the correlated 4 -jet results is determined according to [83] while the information from 2- and 3 -jet events is considered to be independent. The resulting average together with the individual measurements is displayed in fig.19.

The OPAL collaboration [164] has presented an analysis based on global event-shape variables. Here the individual colour factors $C_{A}, T_{F}$ and $C_{F}$ were fitted one at a time together with the strong coupling constant, fixing the values of the other two to the $\mathrm{SU}(3)$ prediction. The results again are in agreement with the expectation from QCD, but as not all independent parameters were varied at the same time, those results are not included into global averages. The values derived for $C_{A} / C_{F}$ and $T_{F} / C_{F}$ are given in table 7 .

As the coefficients of the beta-function depend not only on the number of active fermions but also on the gauge structure of the theory, the colour factors of QCD are also constrained by the running of the strong coupling constant. The complete expressions for the leading coefficients of 


\begin{tabular}{|c|c|c|c|c|c|}
\hline Analysis & \multicolumn{2}{|c|}{ Reference } & $C_{A} / C_{F}$ & $T_{F} / C_{F}$ & Correlation \\
\hline 4-Jet & ALEPH & {$[145]$} & $2.24 \pm 0.40$ & $0.58 \pm 0.29$ & +0.043 \\
\hline 4-Jet & DELPHI & {$[159]$} & $2.12 \pm 0.35$ & $0.46 \pm 0.19$ & \\
\hline 4-Jet & DELPHI & {$[160]$} & $2.32 \pm 0.25$ & $0.27 \pm 0.15$ & -0.242 \\
\hline 4-Jet & L3 & {$[147]$} & $1.95 \pm 0.37$ & $0.23 \pm 0.14$ & \\
\hline 4-Jet & OPAL & {$[161]$} & $2.11 \pm 0.32$ & $0.40 \pm 0.17$ & -0.450 \\
\hline 2 - and 3 -Jet & ALEPH & {$[162]$} & $4.49 \pm 1.35$ & $2.01 \pm 0.99$ & +0.945 \\
\hline individual colour factors & OPAL & {$[164]$} & $2.40 \pm 0.45$ & $0.32 \pm 0.23$ & - \\
\hline running of $\alpha_{s}$ & & {$[69]$} & - unl & ounded cont & $\mathrm{r}-$ \\
\hline$p \bar{p}$ jet production & & {$[163]$} & $2.14 \pm_{0.74}^{0.51}$ & $0.30 \pm_{0.24}^{0.38}$ & \\
\hline Average (4-Jet) & {$[145,16$} & $61,147]$ & $2.20 \pm 0.26$ & $0.32 \pm 0.14$ & -0.220 \\
\hline Average $(2,3,4$-Jet $)$ & as abov & as $[162]$ & $2.22 \pm 0.22$ & $0.33 \pm 0.12$ & +0.007 \\
\hline Global Average & as above & {$[69,163]$} & $2.21 \pm_{0.19}^{0.18}$ & $0.33 \pm_{0.10}^{0.11}$ & \\
\hline
\end{tabular}

Table 7: Measurements of QCD colour factors.

equation eq.(2) are

$$
\beta_{0}=\frac{11}{3} C_{A}-\frac{4}{3} T_{F} n_{f} \quad \text { and } \quad \beta_{1}=\frac{34}{3} C_{A}{ }^{2}-\frac{20}{3} C_{A} T_{F} n_{f}-4 C_{F} T_{F} n_{f} .
$$

For a consistent analysis of the running of $\alpha_{s}$ it is important to take into account, that a variation of the colour factors not only affects the running but also the theoretical predictions on which a measurement of $\alpha_{s}$ at a certain scale is based. Consequently the value extracted for $\alpha_{s}$ in a particular measurement changes with a variation of the colour factors. Taking this into account, an analysis has been performed using the $1993 \mathrm{LEP}$ averages of $R_{Z}$ and $R_{\tau}$ in order to establish for which pairs of colour-factor ratios $C_{A} / C_{F}$ and $T_{F} / C_{F}$ both measurements are compatible with the same coupling constant $\alpha_{s}\left(M_{Z}\right)[69]$.

The information from $R_{Z}$ and $R_{\tau}$ alone cannot be used to measure simultaneously the two colour-factor ratios and the strong coupling constant. It nevertheless restricts the possible combinations of $C_{A} / C_{F}$ and $T_{F} / C_{F}$ to a rather narrow band. The allowed region in the colour factor plane is displayed in fig.20. Also shown are the combined results from all available jetstudies and a measurement obtained from $p \bar{p}$-collider data. The latter is based on the fact that jet-production at the CERN $p \bar{p}$-collider is dominated by gluon-gluon scattering processes. Colour factor information then can be extracted from a comparison of the total jet cross section to the production cross section for secondary quark pairs [163].

Combining the information from the running of $\alpha_{s}$, the collider measurement and the colourfactor result from jet studies and symmetrizing the slightly asymmetric errors yields the global average

$$
\frac{C_{A}}{C_{F}}=2.21 \pm 0.19 \quad \text { and } \quad \frac{T_{F}}{C_{F}}=0.33 \pm 0.11
$$

This result is in good agreement with the expectation for $\mathrm{SU}(3), C_{A} / C_{F}=2.25$ and $T_{F} / C_{F}=0.375$. It confirms the non-abelian nature of QCD $\left(C_{A}>0\right)$ with a significance of more than 11 standard 
deviations. Using as input that quarks come in three colours, the value for $T_{F} / C_{F}$ corresponds to a number of gluons $N_{A}=9 \pm 3$.

\section{The Modified Leading-Log Approximation}

The "modified leading-log approximation" (MLLA) refers to a treatment of higher order QCD effects equivalent to the coherent parton shower picture, where interference effects between subsequent emissions are taken into account through angular ordering. Theoretical predictions in the framework of MLLA $[165,166]$ can be calculated analytically for various processes. The connection between the MLLA-prediction for a multiparton final state, and the actually observable hadronic final state is established by "local parton-hadron duality" (LPHD) which postulates that the cross section on hadron-level is proportional to the one on parton level [167].

\subsection{Momentum Distributions}

Experimental evidence showing the existence of the postulated coherence effects can be obtained from the study of inclusive momentum spectra. Here the consequence of angular ordering is understandable from a simple model, which assumes all emission processes to happen with the same transverse momentum relative to the mother parton. Having the transverse momentum fixed, decreasing emission angles imply increasing total momenta in subsequent emission processes. This forced increase does not exist in the absence coherence. In other words, coherence effects lead to a relative suppression of low momentum partons [165], and via LPHD also to a suppression of low momentum hadrons.

An observable sensitive to this suppression is $\xi=\ln (1 / x)$, where $x=2 p / \sqrt{s}$ is the scaled momentum of a final state particle. Coherence effects lead to a suppression at large values of $\xi$ such that $d \sigma / d \xi$ develops a maximum $\xi^{\star}$. The MLLA and LPHD theoretical prediction is

$$
\frac{1}{\sigma} \frac{d \sigma}{d \xi}=K_{\mathrm{LPHD}} \cdot f_{\mathrm{MLLA}}\left(\xi, E_{c m} / \Lambda_{\mathrm{eff}}\right)
$$

with $K_{\text {LPHD }}$ a phenomenological factor relating the parton-level prediction $f_{\text {MLLA }}$ to the observable cross-section on hadron-level. The parton-level prediction depends only on $\xi$ and the ratio $E_{c m} / \Lambda_{\text {eff }}$, where $E_{c m}$ is the centre-of-mass energy and $\Lambda_{\text {eff }}$ an effective QCD scale parameter. The peak position $\xi^{\star}$ is independent of $K_{\text {LPHD }}$. Its $E_{c m}$-dependence thus allows a direct test of perturbative QCD in the framework of the MLLA, which predicts

$$
\xi^{\star}\left(E_{c m}\right)=\frac{1}{2} \ln \frac{E_{c m}}{2 \Lambda_{\mathrm{eff}}}+K \sqrt{\ln \frac{E_{c m}}{2 \Lambda_{\mathrm{eff}}}}
$$

with

$$
K=\frac{1}{36} \frac{297+2 n_{f}}{\sqrt{297-18 n_{f}}}=\frac{101}{324} \sqrt{3} \quad \text { for } \quad n_{f}=3 .
$$

The choice $n_{f}=3$ active flavours is motivated by the fact that the evolution of the parton shower is dominated by the light flavours. The variation of the peak position is predicted with only one 
free parameter $\Lambda_{\text {eff }}$ which determines the cutoff of the perturbative phase described by a parton shower. As this cutoff is expected to grow with the mass of the final state hadrons the peak position $\xi^{\star}$ should shift to smaller values (larger momenta) for heavier particles.

It is instructive to compare the full MLLA-prediction to a simple analytical model introduced in reference [165]. The expressions obtained there for the $\xi$-distribution can be summarized through

$$
\frac{d \sigma}{d \xi}=1+\alpha_{s} \xi\left(\ln \frac{E_{c m}}{2 \Lambda_{\mathrm{eff}}}-\frac{\xi}{k}\right) .
$$

For $\alpha_{s}=0$ one obtains a simple fragmentation model without gluon emission in the perturbative phase, for $\alpha_{s}>0$ the parameter $k$ distinguishes between different types of parton shower models. The value $k=2$ is characteristic for an incoherent parton shower without angular ordering, $k=1$ corresponds to a coherent parton shower with angular ordering. For $\alpha_{s}>0$ the spectrum exhibits a maximum $\xi^{\star}$, which evolves with energy according to

$$
\xi^{\star}\left(E_{c m}\right)=\frac{k}{2} \ln \frac{E_{c m}}{2 \Lambda_{\mathrm{eff}}} .
$$

As function of $\ln E_{c m}$ it varies with a slope of $S=E_{c m} d \xi^{\star} / d E_{c m}=1 / 2$ for a coherent parton shower and with a slope $S=1$ for an incoherent one. The same slope of $S=1$ is expected from simple phase-space suppression of low momentum massive particles, which due to energy conservation cuts in at a fixed fractional momentum $x_{c u t}=m_{e f f} / E_{c m}$.

Measurements of the $\xi$-distribution obtained at $E_{c m}=91.2 \mathrm{GeV}$ for identified charged and neutral particles are displayed in fig.22 and fig.23, respectively. Also shown is the inclusive distribution for all charged particles. The predicted maximum is clearly visible and the distributions are well described by the MLLA+LPHD theoretical prediction. Using the parameters $\Lambda_{\text {eff }}$ determined at $E_{c m}=91.2 \mathrm{GeV}$, fig.24 demonstrates that the energy evolution of the peak position $\xi^{\star}$ for neutral particles is predicted correctly by QCD without additional free parameters.

A similar analysis can be performed for the inclusive distributions of all charged particles. Where the peak position is not given in the reference it was determined from the published $x$ - or $\xi$-distributions by fitting the experimental data around the maximum to the functional form

$$
\frac{d \sigma}{d x} \sim x^{a}(1-x)^{b} \exp \left(-c \ln (x)^{2}\right)
$$

This ansatz combines a gaussian shape $[165,166]$ which the $\xi$-distribution exhibits in the vicinity of the maximum with the usual power-law behaviour $(1-x)^{b}$ observed for fragmentation functions in the limit $x \rightarrow 1$. The results are collected in table 8 and displayed in fig. 25 . The errors cover both the experimental errors, which were taken to be bin-to-bin errors, and the systematic uncertainty due to the choice of the fit interval. All intervals covering at least six data points around the one with the largest cross section where considered if the confidence level of the fit was above $C L=0.1$. The weighted average of the accepted results was taken as the nominal peak position, the RMS-spread as the systematic error. The energy evolution is in good agreement with the MLLA-expectation and clearly incompatible with the assumption of an incoherent parton shower or a simple phase-space model.

Finally studying the mass dependence of the peak position for identified particles at the $Z$ peak, fig.26, one clearly sees the expected behaviour of $\xi^{\star}$ becomimg smaller for increasing particle 


\begin{tabular}{|c|c|ll|}
\hline$\sqrt{s} / \mathrm{GeV}$ & $\xi^{*}$ & \multicolumn{2}{|c|}{ Reference } \\
\hline 14. & $2.353 \pm \mathbf{0 . 0 4 3}$ & TASSO & {$[171]$} \\
22. & $2.651 \pm \mathbf{0 . 0 4 1}$ & TASSO & {$[171]$} \\
29. & $2.771 \pm \mathbf{0 . 0 1 5}$ & MARK II & {$[172]$} \\
29. & $2.866 \pm \mathbf{0 . 0 6 0}$ & TPC $/ 2 \gamma$ & {$[173]$} \\
35. & $3.063 \pm \mathbf{0 . 0 2 4}$ & TASSO & {$[171]$} \\
35. & $2.929 \pm \mathbf{0 . 0 7 2}$ & CELLO & {$[174]$} \\
44. & $3.120 \pm \mathbf{0 . 0 5 4}$ & TASSO & {$[171]$} \\
55. & $3.147 \pm \mathbf{0 . 0 9 3}$ & AMY & {$[175]$} \\
$\mathbf{9 1 . 2}$ & $3.618 \pm \mathbf{0 . 0 2 8}$ & ALEPH & {$[176]$} \\
$\mathbf{9 1 . 2}$ & $3.67 \pm \mathbf{0 . 1 0}$ & DELPHI & {$[177]$} \\
91.2 & $3.71 \pm \mathbf{0 . 0 5}$ & L3 & {$[178]$} \\
$\mathbf{9 1 . 2}$ & $3.603 \pm \mathbf{0 . 0 4 2}$ & OPAL & {$[168]$} \\
\hline
\end{tabular}

Table 8: Centre-of-mass energy dependence of the peak positions $\xi^{*}$ measured for the inclusive charged particle distribution. For all lower energy data the peak position was fitted to the published spectra.

mass. The qualitative behaviour can be decribed as $\xi^{\star}=\ln \left(m_{0} / m\right)$ with two distinct parameters $m_{0}$ for mesons and baryons. The numerical $\xi^{\star}$-values are listed in table 9 . Where not available from the original reference it was determined as described above.

\subsection{Charged Particle Multiplicities}

Another piece of experimental evidence comes from the measurement of charged particle multiplicities. Although the average multiplicity cannot be calculated perturbatively, its energy evolution is predicted in the framework of MLLA and LPHD [183] to be coupled to the running of the strong coupling constant. The prediction is

$$
<n_{c h}\left(E_{c m}\right)>=K_{\mathrm{LPHD}} \cdot \alpha_{s}^{b}\left(E_{c m}\right) \cdot \exp \left(\frac{a}{\sqrt{\alpha_{s}\left(E_{c m}\right)}}\right)
$$

with

$$
a=\sqrt{6 \pi} \frac{12}{33-2 n_{f}} \quad \text { and } \quad b=\frac{297+22 n_{f}}{1188-72 n_{f}}
$$

and one free parameter $K_{\mathrm{LPHD}}$. Studying ratios of expectation values the unknown constant $K_{\text {LPHD }}$ cancels. The first non-trivial case is the second binomial moments $R_{2}$ with a predicted energy dependence $[184,185]$

$$
R_{2}=\frac{\langle n(n-1)>Q C D}{\langle n\rangle^{2}} \frac{11}{8}\left(1-c \sqrt{\alpha_{s}\left(E_{c m}\right)}\right) \quad \text { with } \quad c=\frac{1}{\sqrt{6 \pi}} \frac{4455-40 n_{f}}{1782} .
$$

Experimental data for the mean charged particle multiplicity $\langle n\rangle$ and the second binomial moment are listed in table 10 . The numbers refer to the convention where all particles with a 


\begin{tabular}{|c|l|c|ll|}
\hline Particle & Mass/GeV & $\xi^{*}$ & \multicolumn{2}{|c|}{ Reference } \\
\hline$\pi^{0}$ & 0.135 & $4.11 \pm \mathbf{0 . 1 8}$ & L3 & {$[178]^{*}$} \\
$\pi^{0}$ & 0.135 & $3.96 \pm \mathbf{0 . 1 3}$ & L3 & {$[170]^{*}$} \\
$\pi^{ \pm}$ & 0.140 & $3.81 \pm \mathbf{0 . 0 2}$ & OPAL & {$[169]^{*}$} \\
all charged & 0.22 & $3.618 \pm \mathbf{0 . 0 2 8}$ & ALEPH & {$[176]^{*}$} \\
all charged & 0.22 & $3.67 \pm \mathbf{0 . 1 0}$ & DELPHI & {$[177]^{*}$} \\
all charged & 0.22 & $3.71 \pm \mathbf{0 . 0 5}$ & L3 & {$[178]^{*}$} \\
all charged & 0.22 & $3.603 \pm \mathbf{0 . 0 4 2}$ & OPAL & {$[168]^{*}$} \\
$K^{ \pm}$ & 0.494 & $2.63 \pm \mathbf{0 . 0 4}$ & OPAL & {$[169]^{*}$} \\
$K_{s}^{0}$ & 0.498 & $2.63 \pm \mathbf{0 . 0 4}$ & ALEPH & {$[179]^{*}$} \\
$K_{s}^{0}$ & 0.498 & $2.62 \pm \mathbf{0 . 1 1}$ & DELPHI & {$[177]^{*}$} \\
$K_{s}^{0}$ & 0.498 & $2.89 \pm \mathbf{0 . 0 5}$ & L3 & {$[170]^{*}$} \\
$K_{s}^{0}$ & 0.498 & $2.91 \pm \mathbf{0 . 0 4}$ & OPAL & {$[180]^{*}$} \\
$\eta$ & 0.547 & $2.60 \pm \mathbf{0 . 1 5}$ & L3 & {$[181]$} \\
$\eta$ & 0.547 & $2.52 \pm \mathbf{0 . 1 0}$ & L3 & {$[170]^{*}$} \\
$p \bar{p}$ & 0.938 & $3.00 \pm \mathbf{0 . 0 9}$ & OPAL & {$[169]^{*}$} \\
$\Lambda \bar{\Lambda}$ & 1.116 & $2.67 \pm \mathbf{0 . 1 4}$ & ALEPH & {$[179]^{*}$} \\
$\Lambda \bar{\Lambda}$ & 1.116 & $2.82 \pm \mathbf{0 . 2 5}$ & DELPHI & {$[177]^{*}$} \\
$\Lambda \bar{\Lambda}$ & 1.116 & $2.83 \pm \mathbf{0 . 1 3}$ & L3 & {$[170]^{*}$} \\
$\Lambda \bar{\Lambda}$ & 1.116 & $2.77 \pm \mathbf{0 . 0 5}$ & OPAL & {$[182]^{*}$} \\
$\Xi^{-}$ & 1.321 & $2.57 \pm \mathbf{0 . 1 1}$ & OPAL & {$[182]^{*}$} \\
\hline
\end{tabular}

Table 9: Measured peak positions $\xi^{*}$ as function of the particle mass. The effective mass for the inclusive measurement of all charged particles is taken as the weighted average of the Pion, Kaon and Proton mass, with the weights given by the average multiplicity of the respective particle type as measured [169] or estimated by the Jetset Monte-Carlo. Numbers from the starred references are displayed in fig. 26 .

mean lifetime below $1 \mathrm{~ns}$ are forced to decay while the others are assumed to be absolutely stable. In most cases the second binomial moment $R_{2}$ is not explicitly given in the respective reference. In those cases it was inferred from mean value $\langle n\rangle$ and dispersion $D$ of the distribution which are related to $R_{2}$ through $R_{2}=1+(D /\langle n\rangle)^{2}-1 /\langle n\rangle$. If $\langle n\rangle, D$ and $\langle n\rangle / D$ are given the correlation between $\langle n\rangle$ and $D$ was estimated from these three numbers and propagated into the error of $R_{2}$. If only two of the three quantities were available the correlation was assumed to be such that it maximizes the error of $R_{2}$.

Comparing the LEP measurements to lower energy results one finds excellent agreement between experimental data and the QCD expectation. The energy dependence of the mean charged particle multiplicity is perfectly described by fixing $\alpha_{s}\left(M_{Z}\right)=0.118$ and adjusting only the phenomenological parameter $K_{\mathrm{LPHD}}$. The results are displayed in fig.27. Also shown is the energy dependence expected for the second binomial moment. While the data are in clear disagreement with the leading order QCD prediction, the bulk of the higher order corrections is taken into account when including the correction proportional to $\sqrt{\alpha_{s}}$. Still, some discrepancies remain which may be due to missing perturbative higher orders or to non-perturbative effects. It is intesting to note that the data are well reproduced by a full simulation as implemented e.g. in 


\begin{tabular}{|c|c|c|c|c|c|c|}
\hline$\sqrt{s} / \mathrm{GeV}$ & $<n>$ & $D$ & $<n>/ D$ & $R_{2}$ & \multicolumn{2}{|c|}{ Reference } \\
\hline 12.0 & $8.4 \pm 0.7$ & - & $2.86 \pm 0.25$ & $1.003 \pm 0.031$ & JADE & {$[186]$} \\
\hline 12.3 & $8.7 \pm 0.6$ & - & $2.75 \pm 0.26$ & $1.017 \pm 0.033$ & PLUTO & {$[187]$} \\
\hline 14.0 & $9.3 \pm 0.41$ & $3.07 \pm 0.28$ & $3.03 \pm 0.31$ & $1.0014 \pm 0.0245$ & TASSO & [188] \\
\hline 17.0 & $9.4 \pm 0.7$ & 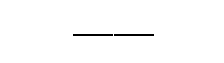 & $2.4 \pm 0.4$ & $1.067 \pm 0.065$ & PLUTO & {$[187]$} \\
\hline 22.0 & $11.2 \pm 1.0$ & - & $2.9 \pm 0.9$ & $1.030 \pm 0.082$ & PLUTO & {$[187]$} \\
\hline 22.0 & $11.3 \pm 0.47$ & $3.55 \pm 0.36$ & $3.19 \pm 0.36$ & $1.010 \pm 0.025$ & TASSO & [188] \\
\hline 27.6 & $12.0 \pm 0.8$ & . & $2.9 \pm 0.4$ & $1.036 \pm 0.038$ & PLUTO & {$[187]$} \\
\hline 29 . & $12.8 \pm 0.6$ & s & — & 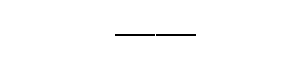 & $\mathrm{TPC} / 2 \gamma$ & {$[189]$} \\
\hline 29 . & $12.87 \pm 0.30$ & $3.67 \pm 0.18$ & $3.51 \pm 0.18$ & $1.0036 \pm 0.0097$ & HRS & {$[190]$} \\
\hline 30.0 & $13.1 \pm 0.7$ & & $3.05 \pm 0.16$ & $1.031 \pm 0.015$ & JADE & {$[186]$} \\
\hline 30.6 & $12.3 \pm 0.8$ & - - & $2.81 \pm 0.15$ & $1.044 \pm 0.019$ & PLUTO & {$[187]$} \\
\hline 34.8 & $13.59 \pm 0.46$ & $4.14 \pm 0.39$ & $3.28 \pm 0.33$ & $1.019 \pm 0.022$ & TASSO & [188] \\
\hline 35 . & $13.6 \pm 0.7$ & 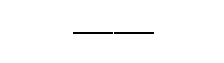 & $3.20 \pm 0.15$ & $1.024 \pm 0.013$ & JADE & {$[186]$} \\
\hline 43.6 & $15.08 \pm 0.47$ & $4.59 \pm 0.37$ & $3.28 \pm 0.28$ & $1.026 \pm 0.019$ & TASSO & {$[188]$} \\
\hline 52. & $15.99 \pm 0.23$ & 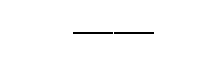 & & & TOPAZ & [191] \\
\hline 55. & $16.85 \pm 0.27$ & 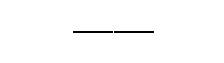 & 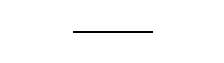 & - & TOPAZ & [191] \\
\hline 57. & $17.19 \pm 0.49$ & $5.03 \pm 0.26$ & $3.42 \pm 0.04$ & $1.0274 \pm 0.0084$ & AMY & {$[192]$} \\
\hline 91.2 & $20.85 \pm 0.24$ & $6.34 \pm 0.11$ & $3.29 \pm 0.06$ & $1.0444 \pm 0.0033$ & ALEPH & {$[193]$} \\
\hline 91.2 & $20.71 \pm 0.77$ & $6.28 \pm 0.43$ & $3.30 \pm 0.20$ & $1.044 \pm 0.015$ & DELPHI & {$[194]$} \\
\hline 91.2 & $21.40 \pm 0.43$ & $6.49 \pm 0.20$ & $3.30 \pm 0.11$ & $1.0452 \pm 0.0074$ & L3 & {$[195]$} \\
\hline 91.2 & $20.79 \pm 0.52$ & - - & & & OPAL & {$[196]$} \\
\hline 91.2 & $20.1 \pm 1.3$ & 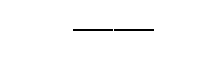 & & & MARK II & {$[197]$} \\
\hline
\end{tabular}

Table 10: Measurements of mean value $\langle n>$, Dispersion $D,<n>/ D$ and second binomial moment $R_{2}$ of the charged particle multiplicity distribution in hadronic events for centre-of-mass energies above $\sqrt{s}=10 \mathrm{GeV}$. The errors are the total statistical and systematic errors. For reference [187] the results quoted at $\sqrt{s}=12.3$ and $30.6 \mathrm{GeV}$ were combined from measurements at $\sqrt{s}=12$ and $13 \mathrm{GeV}$ and $\sqrt{s}=30.2,30.7$ and $31.3 \mathrm{GeV}$, respectively. The numbers for $\langle n\rangle$ were corrected for $V^{0}$-decays, using a linear fit as function of $\sqrt{s}$ to the shifts quoted in [186]. The ratios $\langle n\rangle / D$ given in [187] are assumed to be unaffected by this correction. 
the LUND parton-shower model.

\section{The Structure of the Parton-Shower}

The evolution of an initial quark-antiquark pair into a multihadron final state is well understood to proceed via a parton shower, where an initial four-momentum is distributed on a large number of final state particles in a self-similar cascading process. Experimental evidence corroborating this picture is obtained e.g. from the charged particle multiplicity distribution. Based on rather general assumptions one expects from a cascading model that its shape should be described by a log-normal distribution [198, 199], which is in good agreement with the experimental findings [193, 195]. Another piece of evidence comes from the study of intermittency, i.e. non-poissonian fluctuations of particle multiplicities in restricted phase space intervals. Here the assumption of a self similar cascading mechanism leads to the prediction, that the size of the fluctuations as measured by factorial moments should grow proportionally to a negative power of the size of the phase space interval [200]. Experimental results [201, 202, 203] confirm this behaviour. A quantitative formulation of the parton shower picture exists in the framework of the MLLA. Further information about its structure beyond establishing the self-similarity of the cascade and the angular-ordering property can be obtained e.g. from the study of isolated hard photons or subjet multiplicities.

\subsection{Isolated Photons}

One probe to the early parton showering stage are high energy isolated photons in hadronic events. Being emitted with a large momentum transfer those photons test the short distance properties of the multiparton system constituting the parton shower. For a given formulation of the parton shower in terms of gluon radiation from quarks and gluons also the properties of a photon emitted in the cascade are fixed. As both are massless vector particles, the only difference between a photon and a gluon is, that the former couples to the electric charge of the quarks and the latter to the colour charge. There are no new free parameters. As the photon is blind to colour charges, once emitted it penetrates without further interaction the complicated colour fields of the parton shower, thereby allowing to "see" the partonic cascade directly.

Experimental results are given in $[204,205,206]$. Figure 28 shows how a measurement of the isolated photon rate as function of a resolution parameter $y_{c u t}$ compares to various partonshower models. One finds that for large isolation parameters $y_{c u t}>0.1$ the production rate is reasonably well described by the JETSET and HERWIG models and slightly overestimated by ARIADNE. For small resolution parameters the picture changes. There both ARIADNE and HERWIG provide a good description of the data while JETSET underestimates the photon rate. As the various parton-shower models only differ in the way how next-to-leading logarithmic effects are implemented, these findings demonstrate that the detailed structure of the parton cascade can be studied with isolated hard photons.

While the JETSET model appears to be at variance with the data, this does not mean that the parton shower implementation of this model is conceptually inferior to the other approaches. One has to keep in mind that the model parameters are adjusted such that the overall properties 
of hadronic events as measured by the final state hadrons are well reproduced by the combined parton showering and hadronization stage. Perturbative and non-perturbative effects thus are not disentangled. Isolated photons on the other hand, which see through the hadronization phase, offer a way to decouple the parameters describing the two regimes.

\subsection{Subjet Multiplicities}

Another approach to test the structure of the parton shower is by means of "subjet multiplicities". In a first step a hadronic final state is clustered with a jet-resolution parameter $y_{1}$ into a well defined number of initial jets. Then the same event is looked at with a different resolution parameter $y_{0}<y_{1}$ and the number of clusters studied as function of $y_{0}$. The increase in the number of jets when lowering the jet-resolution parameter is predicted in perturbative QCD with a resummation of leading and next-to-leading logarithms [207].

Let $M_{3}$ and $M_{2}$ be the jet-multiplicities at a resolution $y_{0}$ when the initial multiplicities at $y_{1}$ are 3 and 2 jets, respectively. Figure 29 shows experimental results [208] for the ratio of the additional jets $\left(M_{3}-3\right) /\left(M_{2}-2\right)$ as function of $y_{0}$. After a sharp rise when going to smaller values $y_{0}$ the ratio starts to level off around $y_{0}=4 \cdot 10^{-4}$. The rise is well described by the perturbative QCDprediction including an all orders resummation of leading and next-to-leading logarithms. The importance of resumming those logarithms is illustrated by comparing the resummed prediction to a fixed order calculation which fails to describe the data. The levelling off of the experimental data at very small values $y_{0}$ is interpreted as being due to non-perturbative effects which exhibit a universal behaviour independent of the number of jets at the hard scale $y_{1}$. It is therefore illustrative to confront the data with model calculations that take non-perturbative effects into account. Using models that have been tuned to reproduce the data, one finds that the coherent parton shower models with cluster or string hadronization successfully reproduce the experimental results over the full $y_{0}$-range. The models based on an incoherent parton shower and independent fragmentation only reproduce the qualitative trend, but fail to describe the data quantitatively. It appears that non-perturbative effects indeed account for the levelling off of the measurements, independent of the details of the hadronization mechanism. The discrepancies between coherent and non-coherent parton shower models are of similar size as the differences between resummed and the fixed order QCD calculations, which again supports the picture of a coherent parton shower to describe higher order perturbative effects.

\section{The Hadronization Process}

The properties of the perturbative evolution are well described within QCD. In contrast to this the understanding of the non-perturbative hadron formation is still in a more exploratory state, guided by phenomenological models and asymptotic predictions.

\subsection{Bose-Einstein Correlations}

A possibility to measure the size of the particle emitting source is through Bose-Einstein correlations of identical bosons. Symmetrization of the wave function implies that the production 
of identical particles is enhanced if they are close together in phase space. The correlation function is usually parametrized through

$$
R\left(\vec{p}_{1}, \vec{p}_{2}\right)=\frac{\sigma\left(\vec{p}_{1}, \vec{p}_{2}\right)}{\sigma\left(\vec{p}_{1}\right) \sigma\left(\vec{p}_{2}\right)}=1+\lambda \exp \left(-r^{2} Q^{2}\right) \quad \text { with } \quad Q^{2}=\left(p_{1}-p_{2}\right)^{2}-4 m^{2},
$$

where $r$ denotes the radius of the particle emitting source and $\lambda$ a parameter that depends on the dynamic properties of the source. Uncorrelated emission implies $\lambda=1$. The $Q^{2}$-dependence of the correlation function thus can be used to measure the size of the particle emitting region.

Experimental results from $[209,210]$ for same charge pions and $K_{s}^{0}$-mesons are shown in figs.30 and 31, respectively. Additional measurements are available from [211, 212, 213]. The enhancement at small $Q^{2}$ is clearly visible and well described by the above parametrization. A compilation of results obtained by the different LEP experiments taken from [210] is displayed in fig.32. The inner line shows the $68 \%$ and the outer one the $90 \%$ confidence-level contour based on the statistical errors only. The comparatively large spread between the individual results may be explained by different correction procedures for non-interfering pairs and different reference samples. Mainly affected is the amplitude $\lambda$ of the enhancement term. The measurements of the source radius appear to be much more consistent, suggesting a universal value around $0.8 \pm 0.2 \mathrm{fm}$. Via the uncertainty relation this radius translates into an energy scale governing the hadronization process of $Q_{h a d r}=0.24 \pm 0.06 \mathrm{GeV}$. Not surprisingly, this value is of the same order of magnitude as the QCD-scale parameter $\Lambda$, i.e. the energy scale where a diverging running coupling constant signals the breakdown of perturbative QCD.

\subsection{Strangeness Correlations}

The finding that the non-perturbative hadron formation process is related to an energy scale around $Q_{h a d r}=0.24 \mathrm{GeV}$ has an interesting phenomenological consequence in the context of parton shower models. As discussed before, those models are based on perturbative QCD down to an energy scale of typically $Q_{0}=1 \mathrm{GeV}$. According to LPHD the details of the hadronization model should not matter for the description of the hadronic final state, but this requires the perturbative phase to be modeled completely, i.e. to the point $Q_{\text {hadr }}$ where non-perturbative dynamics takes over. As this is not the case for the current models the hadronization step has to bridge the gap between $Q_{0}$ and $Q_{h a d r}$, and it is interesting to study experimentally how e.g. string and cluster hadronization compare in this respect.

One way to address this question is to study correlations between strange baryons $(\Lambda \bar{\Lambda})$, measured e.g. by the rapidity difference of the particles with respect to the Thrust axis. Such $\Lambda \bar{\Lambda}$-pairs trace secondary strange quarks produced in the hadronization step and thus allow to probe the dynamics of multiparticle production: a string-like mechanism oriented preferentially along the direction of the quark-antiquark pair from the primary $Z$-decay would result in a wider distribution for the rapidity differences than an isotropic cluster decay model. Experimental results are published in $[214,215]$. Figure 33 shows that the actual measurements clearly favour the chain-like production mechanism implemented in the Lund-string picture over an isotropic cluster decay. In fact, the rapidity differences are even larger than expected from the simplest version of the string-model, where the final state $\Lambda \bar{\Lambda}$-pair is produced as two neighbouring particles 
in phase space. The data rather support the assumption that in most cases an additional pion is produced between the two baryons ("popcorn model" [216]).

\subsection{Differences between Quark and Gluon Jets}

Asymptotically one expects the multiplicity of a gluon initiated jet to be a factor $C_{A} / C_{F}=2.25$ larger than the multiplicity of a quark jet [217, 218], i.e. a multiplicity proportional to the square of the colour charge which determines the amount of radiation from the respective parton. More recent theoretical studies have shown that due to coherence effects in the parton shower the enhancement factor is much closer to unity [207]. Other observable consequences of the fact that a gluon radiates more than a quark are a softer fragmentation function and a larger angular width of a gluon jet.

The so far most convincing experimental results concerning differences between quark and gluon jets come from the study of Y-shaped symmetric 3-jet configurations, where two lower energy jets recoil against a high energy jet. With an opening angle of $150^{\circ}$ between the high energy jet and the two low energy ones the high energy jet is a quark jet in about $97 \%$ of all cases. Without further tagging the sample of all low energy jets contains about equal amounts of quark and gluon jets of the same energy. Using lifetime information or a lepton-tag to identify the quark jets allows to select an unbiased gluon-jet enriched sample without changing the kinematical properties of the jets. From those two samples which have different quark-gluon composition the properties of quarks and gluons can be unfolded.

Results are given in $[219,220,221,222]$. The experimental findings are in qualitative agreement with the QCD expectation. For the chosen kinematical configuration the measured ratio of the charged particle multiplicities of quark and gluon jets, $R_{\mathrm{ch}}=<n_{\mathrm{ch}}($ Gluon $\left.)\right\rangle /<n_{\mathrm{ch}}($ Quark $\left.)\right\rangle$, is found to be $R_{\mathrm{ch}}=1.27 \pm 0.04($ stat $) \pm 0.06($ syst $)[220], R_{\mathrm{ch}}=1.17 \pm 0.03($ stat $)$ [221] and $R_{\text {ch }}=1.19 \pm 0.02($ stat $) \pm 0.03$ (syst) [222]. The ratio is significantly different from unity, although still far away from the asymptotic expectation $R_{\text {ch }}=2.25$. Consistent with the higher multiplicity, the fragmentation function of gluon jets is found to be softer than for quark jets. Studying the energy fraction contained in a fixed angular cone around the jet axis one finds a smaller value for gluon jets than for quark jets [220,222], which demonstrates that gluon jets indeed are less collimated than quark jets.

\subsection{The "String Effect"}

Another observable sensitive to coherence effects in soft-gluon emission processes is the particle flow in inter-jet regions. From the Lund-string model, which creates particles from the breakup of a colour flux-tube stretching from the quark to the gluon and from the gluon to the antiquark, one expects larger particle flows between the quarks and the gluon than between the two quarks. The same qualitative effect is predicted from colour coherence in the framework of the MLLA [223, 224]. It is also reproduced by coherent parton shower models.

Experimentally the string effect can be studied by exploiting the same technique that is used in the study of quark-gluon differences. Starting from symmetric configurations where one of the two low energetic jets is identified as a quark jet with a lifetime or lepton tag, one can directly 
measure the particle flow between a low energy quark or gluon and the high energy quark. As the high energy quark has the same angular separation from both of the two lower energy partons the quark-gluon the quark-antiquark system are kinematically equivalent. The differences found experimentally $[225,221]$ thus confirm in a model independent way the existence of the string effect for a fixed kinematic configuration.

A wider range of jet-topologies can be explored by comparing the particle flow between the two quarks in $q \bar{q} g$ events to the one in $q \bar{q} \gamma$ events. In the former case the quark-antiquark system again can be identified by lepton or lifetime tag, in the latter one it is immediately given by the whole hadronic system. Due to the string effect the particle flow between the quark-antiquark system measured in $q \bar{q} g$ events should be suppressed compared to the one in $q \bar{q} \gamma$ events. The experimental results agree with this expectation $[226,221]$.

\section{Summary}

With the high statistics data collected in hadronic $Z$-decays a remarkable understanding of the dynamics of QCD has been achieved, spanning from the hard perturbative regime over the parton showering process to the non-perturbative region of hadron formation.

A compilation of $\alpha_{s}$-determinations from a large variety of reactions and energy-scales shows that all data are compatible with one global average

$$
\alpha_{s}\left(M_{Z}\right)=0.1180 \pm 0.0045
$$

Within the current experimental precision the strong coupling constant has been found to be flavour independent. The LEP average of the most precise single test is

$$
\alpha_{s}(b) / \alpha_{s}(u d s c)=0.999 \pm 0.025
$$

The ratios of the colour factors are measured as

$$
\frac{C_{A}}{C_{F}}=2.21 \pm 0.19 \text { and } \quad \frac{T_{F}}{C_{F}}=0.33 \pm 0.11
$$

showing that the gauge structure of QCD is compatible with the expectation for an unbroken $\mathrm{SU}(3)$ symmetry, $C_{A} / C F=2.25$ and $T_{F} / C_{F}=0.375$. Its non-abelian nature, $C_{A}>0$, is demonstrated with a significance of more than 11 standard deviations.

Experimental evidence exists that the parton showering process proceeds via a self-similar stochastic branching process as implemented in coherent parton shower models. The importance of coherence effects is demonstrated by the energy evolution of inclusive momentum spectra and charged particle multiplicities, both of which are quantitatively described by the modified leadinglog approximation (MLLA) and local parton-hadron duality (LPHD).

The understanding of the hadronization stage still relies on phenomenological models. The Lund string-model successfully describes the dynamics of QCD close to the non-perturbative hadronization scale $Q_{\text {hadr }} \approx 0.2 \mathrm{GeV}$. It is thus well suited to interpolate between the typical cutoff-scale of a parton shower model $Q_{0} \approx 1 \mathrm{GeV}$ and $Q_{\text {hadr }}$, where the final state hadrons are being formed. 


\section{Acknowledgements}

It is a pleasure to thank all colleagues who contributed to this article for their help in collecting and understanding the material presented here. In particular I would like to mention Siggi Bethke, Stefano Catani, Achim Geiser, Giorgio Gratta, Thomas Hebbeker, Thomas Lohse, Peter Mättig, Ramon Miquel, Andreas Ricker, Andreas Seitz, Heinz-Georg Sander, Ron Settles, Torbjörn Sjostrand, Miriam Turner and Bryan Webber. 


\section{Appendix}

\section{A Parameter Adjustments}

The following tables collect optimized parameters sets for the description of hadronic $Z$ decays by the most common event generators. The specific version number for which the individual parameter sets were determined is given together with the reference. If no numerical value is quoted the respective default applies.

\begin{tabular}{|l|l|c|c|c|c|}
\hline JETSET PS & & $7.2 /$ ALEPH $[29]$ & $7.3 /$ L3 $[28]$ & $7.3 /$ L3 $[28]$ & $7.2 /$ OPAL [227] \\
\hline$\Lambda_{\text {LL }}$ & PARJ(81) & 0.318 & 0.30 & 0.29 & 0.29 \\
$Q_{0}$ & PARJ(82) & 1.43 & 1. & 2. & 1.0 \\
$\sigma$ & PARJ(21) & 0.360 & 0.39 & 0.48 & 0.37 \\
A & PARJ(41) & 0.5 & 0.5 & 0.5 & 0.18 \\
B & PARJ(42) & 0.92 & 0.76 & 0.82 & 0.34 \\
c,b fragmentation & MSTJ(11) & 3 & 3 & 3 & - \\
$\varepsilon_{c}$ & PARJ(54) & -0.020 & -0.07 & -0.07 & - \\
$\varepsilon_{b}$ & PARJ(55) & -0.015 & -0.008 & -0.008 & - \\
Bose-Einstein corr. & MSTJ(51) & - & 1 & 1 & - \\
BE parameter & PARJ(92) & - & 2.5 & 2.5 & - \\
\hline
\end{tabular}

Table 11: Optimized parameter sets for the JETSET parton shower model.

\begin{tabular}{|l|l|c|c|c|c|}
\hline HERWIG & & $4.3 /$ ALEPH $[29]$ & $5.0 /$ ALEPH $[29]$ & $5.4 /$ L3 [28] & $3.4 /$ OPAL [227] \\
\hline$\Lambda_{\mathrm{LL}}$ & QCDLAM & 0.099 & 0.179 & 0.17 & 0.110 \\
$M_{g}$ & RMASS(13) & 0.83 & 0.91 & 0.75 & 0.65 \\
$M_{\max }$ & CLMAX & 3.78 & 3.67 & 3.0 & 3.0 \\
\hline
\end{tabular}

Table 12: Optimized parameter sets for the HERWIG model.

\begin{tabular}{|l|l|c|c|c|}
\hline ARIADNE & & $3.1 /$ ALEPH [29] & $3.3 / \mathrm{L3}[28]$ & $3.1 /$ OPAL $[227]$ \\
\hline$\Lambda_{\mathrm{LL}}$ & VAR(1) & 0.212 & 0.22 & 0.20 \\
$P_{\min }^{\mathrm{T}}$ & VAR(3) & 0.90 & - & 1.0 \\
$\sigma$ & PARJ(21) & 0.364 & 0.50 & 0.37 \\
$\mathrm{~A}$ & PARJ(41) & - & 0.65 & 0.18 \\
$\mathrm{~B}$ & PARJ(42) & 0.76 & - & 0.34 \\
c,b fragmentation & MSTJ(11) & 3 & 3 & - \\
$\varepsilon_{\mathrm{c}}$ & PARJ(54) & -0.050 & -0.070 & - \\
$\varepsilon_{\mathrm{b}}$ & PARJ(55) & -0.006 & -0.008 & - \\
Bose-Einstein corr. & MSTJ(51) & - & 1 & - \\
BE parameter & PARJ(92) & - & 2.5 & - \\
\hline
\end{tabular}

Table 13: Optimized parameter sets for the ARIADNE model. 


\begin{tabular}{|l|l|c|}
\hline NLLJET & & $2.0 /$ L3 $[28]$ \\
\hline$\Lambda_{\mathrm{NLL}}$ & LAMBDA & 0.29 \\
$\sigma$ & PARJ(21) & 0.40 \\
$\mathrm{~B}$ & PARJ(42) & 0.70 \\
$\mathrm{c}, \mathrm{b}$ fragmentation & MSTJ(11) & 3 \\
$\varepsilon_{\mathrm{c}}$ & PARJ(54) & -0.07 \\
$\varepsilon_{\mathrm{b}}$ & PARJ(55) & -0.008 \\
Bose-Einstein corr. & MSTJ(51) & 1 \\
BE parameter & PARJ(92) & 2.5 \\
\hline
\end{tabular}

Table 14: Optimized parameters for the NLLJET model.

\begin{tabular}{|l|l|c|}
\hline COJETS & & $6.22 /$ L3 $[28]$ \\
\hline$b_{q}$ & FRALOQ(2) & 43. \\
$b_{g}$ & FRALOG(2) & 100. \\
$d_{q}$ & FRALOQ(4) & 2.1 \\
$d_{g}$ & FRALOG(4) & 2.1 \\
\hline
\end{tabular}

Table 15: Optimized Parameters for the COJETS model.

\begin{tabular}{|l|l|c|c|c|}
\hline JETSET ME & & $7.2 /$ ALEPH $[29]$ & $7.3 /$ L3 $[28]$ & $7.2 /$ OPAL $[228]$ \\
\hline$\Lambda \frac{\text { Mff }}{\text { MS }}$ & PARJ(122) & 0.140 & 0.17 & 0.13 \\
ren. scale $f=\mu^{2} / s$ & PARJ(129) & 0.00136 & 0.003 & 0.002 \\
$y_{\text {min }}$ & PARJ(125) & 0.01 & 0.01 & 0.01 \\
$\sigma$ & PARJ(21) & 0.440 & 0.5 & 0.42 \\
A & PARJ(41) & 1.0 & - & 1.50 \\
B & PARJ(42) & 0.496 & 0.42 & 0.60 \\
c,b fragmentation & MSTJ(11) & 3 & 3 & - \\
$\varepsilon_{\mathrm{c}}$ & PARJ(54) & -0.055 & -0.07 & - \\
$\varepsilon_{\mathrm{b}}$ & PARJ(55) & -0.012 & -0.008 & - \\
Bose-Einstein corr. & MSTJ(51) & - & 1 & - \\
BE parameter & PARJ(92) & - & 2.5 & - \\
\hline
\end{tabular}

Table 16: Optimized parameter sets for the JETSET matrix element model.

\section{B $\quad \alpha_{s}$ Measurements from Event Shape Variables}

A compilation of $\alpha_{s}$-measurements done at SLC and LEP based on individual event shape variables is given below in tables 17, 18, 19, 20 and 21. The starred results are displayed in fig.12 if the theoretical prediction is purely second order, or fig. 13 for improved calculations with resummed leading- and next-to-leading logarithmic corrections. The total error is given for all measurements. Where available from the original reference, also the purely experimental part is given. If not specified otherwise in the respective publications the total error of a result is taken to be the quadratic sum of all experimental and theoretical uncertainties. If several results based on the same theoretical prediction are quoted for one event shape variable in a single publication those results are combined into one number. Details about the treatment of the data in such cases are given below. 


\begin{tabular}{|l|c|c|c|c|c|}
\hline Observable & $\alpha_{s}\left(M_{Z}\right)$ & Total Error & Exp. Error & Reference & Theory \\
\hline$y_{3}(\mathrm{~J}, \mathrm{E})$ & $\mathbf{0 . 1 2 3}$ & $\pm \mathbf{0 . 0 1 0}$ & $\pm \mathbf{0 . 0 0 9}$ & {$[76]^{*}$} & $\mathrm{O}\left(\alpha_{s}^{2}\right)$ \\
$y_{3}(\mathrm{D}, \mathrm{E})$ & $\mathbf{0 . 1 2 5}$ & $\pm \mathbf{0 . 0 1 1}$ & $\pm \mathbf{0 . 0 0 4}$ & {$[77]^{*}$} & $\mathrm{O}\left(\alpha_{s}^{2}\right)$ \\
$y_{3}(\mathrm{M}, \mathrm{E} 0)$ & $\mathbf{0 . 1 1 8}$ & $\pm \mathbf{0 . 0 1 3}$ & $\pm \mathbf{0 . 0 0 4}$ & {$[77]^{*}$} & $\mathrm{O}\left(\alpha_{s}^{2}\right)$ \\
$y_{3}(\mathrm{M}, \mathrm{E})$ & $\mathbf{0 . 1 2 8}$ & $\pm \mathbf{0 . 0 2 1}$ & $\pm \mathbf{0 . 0 0 4}$ & {$[77]^{*}$} & $\mathrm{O}\left(\alpha_{s}^{2}\right)$ \\
$y_{3}(\mathrm{M}, \mathrm{P} 0)$ & $\mathbf{0 . 1 1 4}$ & $\pm \mathbf{0 . 0 0 8}$ & $\pm \mathbf{0 . 0 0 4}$ & {$[77]^{*}$} & $\mathrm{O}\left(\alpha_{s}^{2}\right)$ \\
$y_{3}(\mathrm{M}, \mathrm{P})$ & $\mathbf{0 . 1 1 6}$ & $\pm \mathbf{0 . 0 0 9}$ & $\pm \mathbf{0 . 0 0 4}$ & {$[77]^{*}$} & $\mathrm{O}\left(\alpha_{s}^{2}\right)$ \\
$y_{3}(\mathrm{G}, \mathrm{E})$ & $\mathbf{0 . 1 0 8}$ & $\pm \mathbf{0 . 0 0 9}$ & $\pm \mathbf{0 . 0 0 4}$ & {$[77]^{*}$} & $\mathrm{O}\left(\alpha_{s}^{2}\right)$ \\
$y_{3}(\mathrm{D}, \mathrm{E})$ & $\mathbf{0 . 1 2 6}$ & $\pm \mathbf{0 . 0 0 7}$ & $\pm \mathbf{0 . 0 0 4}$ & {$[77]^{*}$} & $\mathrm{O}\left(\alpha_{s}^{2}\right)+\mathrm{LL}+\mathrm{NLL}$ \\
\hline
\end{tabular}

Table 17: Compilation of individual $\alpha_{s}$-measurements published by the MARK II [76] and the SLD [77] collaborations.

\begin{tabular}{|l|c|c|c|c|c|}
\hline Observable & $\alpha_{s}\left(M_{Z}\right)$ & Total Error & Exp. Error & Reference & Theory \\
\hline $\mathrm{T}$ & 0.119 & $\pm_{0.029}^{0.018}$ & $\pm \mathbf{0 . 0 0 4}$ & {$[229]^{*}$} & $\mathrm{O}\left(\alpha_{s}^{2}\right)$ \\
$\mathrm{O}$ & 0.186 & $\pm_{0.036}^{0.058}$ & $\pm \mathbf{0 . 0 0 3}$ & {$[229]^{*}$} & $\mathrm{O}\left(\alpha_{s}^{2}\right)$ \\
$\mathrm{C}$ & 0.112 & $\pm_{0.030}^{0.021}$ & \pm 0.004 & {$[229]^{*}$} & $\mathrm{O}\left(\alpha_{s}^{2}\right)$ \\
$M_{\mathrm{H}, \mathrm{T}}$ & 0.136 & $\pm_{0.017}^{0.015}$ & \pm 0.004 & {$[229]^{*}$} & $\mathrm{O}\left(\alpha_{s}^{2}\right)$ \\
$M_{\mathrm{D}, \mathrm{T}}$ & 0.142 & $\pm_{0.015}^{0.015}$ & $\pm \mathbf{0 . 0 0 4}$ & {$[229]^{*}$} & $\mathrm{O}\left(\alpha_{s}^{2}\right)$ \\
$y_{3}(\mathrm{~J}, \mathrm{E} 0)$ & 0.121 & $\pm_{0.011}^{0.014}$ & $\pm \mathbf{0 . 0 0 4}$ & {$[229]^{*}$} & $\mathrm{O}\left(\alpha_{s}^{2}\right)$ \\
$\mathrm{CEEC}$ & 0.118 & $\pm_{0.008}^{0.011}$ & $\pm \mathbf{0 . 0 0 2}$ & {$[62]^{*}$} & $\mathrm{O}\left(\alpha_{s}^{2}\right)$ \\
$\mathrm{CT}$ & 0.123 & $\pm_{0.010}^{0.015}$ & $\pm \mathbf{0 . 0 0 4}$ & {$[62]^{*}$} & $\mathrm{O}\left(\alpha_{s}^{2}\right)$ \\
$\mathrm{CO}$ & 0.115 & $\pm_{0.009}^{0.012}$ & $\pm \mathbf{0 . 0 0 4}$ & {$[62]^{*}$} & $\mathrm{O}\left(\alpha_{s}^{2}\right)$ \\
$\mathrm{CC}$ & 0.124 & $\pm_{0.012}^{0.010}$ & $\pm \mathbf{0 . 0 0 4}$ & {$[62]^{*}$} & $\mathrm{O}\left(\alpha_{s}^{2}\right)$ \\
$y_{3}(\mathrm{D}, \mathrm{E})$ & 0.1257 & $\pm \mathbf{0 . 0 0 4 7}$ & $\pm \mathbf{0 . 0 0 1 8}$ & {$[78]^{*}$} & $\mathrm{O}\left(\alpha_{s}^{2}\right)+\mathrm{LL}+\mathrm{NLL}$ \\
$\mathrm{T}$ & 0.1263 & $\pm \mathbf{0 . 0 0 7 2}$ & $\pm \mathbf{0 . 0 0 1 3}$ & {$[78]^{*}$} & $\mathrm{O}\left(\alpha_{s}^{2}\right)+\mathrm{LL}+\mathrm{NLL}$ \\
$M_{\mathrm{H}, \mathrm{T}}$ & 0.1243 & $\pm \mathbf{0 . 0 0 7 9}$ & $\pm \mathbf{0 . 0 0 3 4}$ & {$[78]^{*}$} & $\mathrm{O}\left(\alpha_{s}^{2}\right)+\mathrm{LL}+\mathrm{NLL}$ \\
$\mathrm{EEC}$ & 0.126 & $\pm \mathbf{0 . 0 1 1 6}$ & $\pm \mathbf{0 . 0 0 2}$ & {$[79]^{*}$} & $\mathrm{O}\left(\alpha_{s}^{2}\right)+\mathrm{LL}+\mathrm{NLL}$ \\
$R \tau$ & 0.118 & $\pm \mathbf{0 . 0 0 5}$ & & {$[68]^{*}$} & $\mathrm{O}\left(\alpha_{s}^{3}\right)+\mathrm{SVZ}$ \\
$d \sigma / d x$ & 0.127 & $\pm \mathbf{0 . 0 1 1}$ & $\pm \mathbf{0 . 0 0 8}$ & {$[90]^{*}$} & $\mathrm{NLO}$ \\
\hline
\end{tabular}

Table 18: Compilation of individual $\alpha_{s}$-measurements performed by the ALEPH collaboration.

Measurements by the DELPHI collaboration are listed in table 19 . For the results from reference $[80]$ the numbers given with superscript (1) are for the $\ln R$-matching scheme which was declared to be the central matching scheme. The errors were inflated by adding in quadrature the scale and matching errors and the uncertainty due to the choice of the fit range. The superscript (2) attached to the measurements published in [71] indicates that the unweighted average of the results obtained with the two different correction schemes is given. Here the total errors are constructed such, that they touch the extremes reached by the total errors for either of the two schemes. 


\begin{tabular}{|c|c|c|c|c|c|}
\hline Observable & $\alpha_{s}\left(M_{Z}\right)$ & Total Error & Exp. Error & Reference & Theory \\
\hline$T$ & 0.122 & \pm 0.008 & & {$[80]$} & $\mathrm{LL}+\mathrm{NLL}$ \\
\hline$M_{\mathrm{H}, \mathrm{T}}$ & 0.117 & \pm 0.008 & & {$[80]$} & $\mathrm{LL}+\mathrm{NLL}$ \\
\hline$M_{\mathrm{H}, \mathrm{m}}$ & 0.119 & \pm 0.007 & & {$[80]$} & $\mathrm{LL}+\mathrm{NLL}$ \\
\hline$B_{t o t}$ & 0.116 & \pm 0.007 & & {$[80]$} & $\mathrm{LL}+\mathrm{NLL}$ \\
\hline$B_{w}$ & 0.113 & \pm 0.010 & & {$[80]$} & $\mathrm{LL}+\mathrm{NLL}$ \\
\hline $\mathrm{T}$ & 0.122 & $\pm 0.008^{(1)}$ & & {$[80]^{*}$} & $\mathrm{O}\left(\alpha_{s}^{2}\right)+\mathrm{LL}+\mathrm{NLL}$ \\
\hline$M_{\mathrm{H}, \mathrm{T}}$ & 0.123 & $\pm 0.010^{(1)}$ & & {$[80]^{*}$} & $\mathrm{O}\left(\alpha_{s}^{2}\right)+\mathrm{LL}+\mathrm{NLL}$ \\
\hline$M_{\mathrm{H}, \mathrm{m}}$ & 0.124 & $\pm 0.009^{(1)}$ & & {$[80]^{*}$} & $\mathrm{O}\left(\alpha_{s}^{2}\right)+\mathrm{LL}+\mathrm{NLL}$ \\
\hline$B_{\text {tot }}$ & 0.121 & $\pm 0.010^{(1)}$ & & {$[80]^{*}$} & $\mathrm{O}\left(\alpha_{s}^{2}\right)+\mathrm{LL}+\mathrm{NLL}$ \\
\hline$B_{w}$ & 0.110 & $\pm 0.010^{(1)}$ & & {$[80]^{*}$} & $\mathrm{O}\left(\alpha_{s}^{2}\right)+\mathrm{LL}+\mathrm{NLL}$ \\
\hline EEC & 0.134 & $\pm 0.009^{(1)}$ & & {$[80]^{*}$} & $\mathrm{O}\left(\alpha_{s}^{2}\right)+\mathrm{LL}+\mathrm{NLL}$ \\
\hline $\mathrm{T}$ & 0.1230 & $\pm 0.0120^{(2)}$ & & {$[71]^{*}$} & $\mathrm{O}\left(\alpha_{s}^{2}\right)$ \\
\hline 0 & 0.1205 & $\pm_{0.0155}^{0.0245(2)}$ & & {$[71]^{*}$} & $\mathrm{O}\left(\alpha_{s}^{2}\right)$ \\
\hline C & 0.1220 & $\pm 0.0110^{(2)}$ & & {$[71]^{*}$} & $\mathrm{O}\left(\alpha_{s}^{2}\right)$ \\
\hline$M_{\mathrm{H}, \mathrm{T}}$ & 0.1215 & $\pm_{0.0065}^{0.0085(2)}$ & & {$[71]^{*}$} & $\mathrm{O}\left(\alpha_{s}^{2}\right)$ \\
\hline$M_{\mathrm{D}, \mathrm{T}}$ & 0.1185 & $\pm_{0.0055}^{0.0075(2)}$ & & {$[71]^{*}$} & $\mathrm{O}\left(\alpha_{s}^{2}\right)$ \\
\hline EEC & 0.1155 & $\pm_{0.0085}^{0.0075(2)}$ & & {$[71]^{*}$} & $\mathrm{O}\left(\alpha_{s}^{2}\right)$ \\
\hline AEEC & 0.1075 & $\pm_{0.0045}^{0.0075(2)}$ & & {$[71]^{*}$} & $\mathrm{O}\left(\alpha_{s}^{2}\right)$ \\
\hline$y_{3}(\mathrm{~J}, \mathrm{E})$ & 0.1140 & $\pm_{0.0080}^{0.0090(2)}$ & & {$[71]^{*}$} & $\mathrm{O}\left(\alpha_{s}^{2}\right)$ \\
\hline AEEC & 0.106 & $\pm_{0.004}^{0.005}$ & \pm 0.003 & {$[230]$} & $\mathrm{O}\left(\alpha_{s}^{2}\right)$ \\
\hline$y_{3}(\mathrm{~J}, \mathrm{E})$ & 0.114 & \pm 0.013 & \pm 0.003 & {$[231]$} & $\mathrm{O}\left(\alpha_{s}^{2}\right)$ \\
\hline$d \sigma / d x$ & 0.118 & \pm 0.005 & \pm 0.002 & {$[89]$} & ERT-JETSET \\
\hline
\end{tabular}

Table 19: Compilation of individual $\alpha_{s}$-measurements published by the DELPHI collaboration.

\begin{tabular}{|l|c|c|c|c|c|}
\hline Observable & $\alpha_{\boldsymbol{s}}\left(M_{Z}\right)$ & Total Error & Exp. Error & Reference & Theory \\
\hline $\mathrm{T}$ & $\mathbf{0 . 1 2 3 3}$ & $\pm \mathbf{0 . 0 0 8 1}$ & $\pm \mathbf{0 . 0 0 2 5}$ & {$[81]^{*}$} & $\mathrm{O}\left(\alpha_{s}^{2}\right)+\mathrm{LL}+\mathrm{NLL}$ \\
$M_{\mathrm{H}, \mathrm{T}}$ & $\mathbf{0 . 1 2 2 8}$ & $\pm \mathbf{0 . 0 0 5 4}$ & $\pm \mathbf{0 . 0 0 1 3}$ & {$[81]^{*}$} & $\mathrm{O}\left(\alpha_{s}^{2}\right)+\mathrm{LL}+\mathrm{NLL}$ \\
$\mathrm{EEC}$ & $\mathbf{0 . 1 3 2 6}$ & $\pm \mathbf{0 . 0 0 6 2}$ & $\pm \mathbf{0 . 0 0 1 1}$ & {$[81]^{*}$} & $\mathrm{O}\left(\alpha_{s}^{2}\right)+\mathrm{LL}+\mathrm{NLL}$ \\
$B_{\text {tot }}$ & $\mathbf{0 . 1 2 5 8}$ & $\pm \mathbf{0 . 0 0 8 1}$ & $\pm \mathbf{0 . 0 0 2 0}$ & {$[81]^{*}$} & $\mathrm{O}\left(\alpha_{s}^{2}\right)+\mathrm{LL}+\mathrm{NLL}$ \\
$B_{w}$ & $\mathbf{0 . 1 1 6 5}$ & $\pm \mathbf{0 . 0 0 5 9}$ & $\pm \mathbf{0 . 0 0 2 2}$ & {$[81]^{*}$} & $\mathrm{O}\left(\alpha_{s}^{2}\right)+\mathrm{LL}+\mathrm{NLL}$ \\
$\left\langle n_{\text {jet }}\right\rangle$ & $\mathbf{0 . 1 3 1 5}$ & $\pm \mathbf{0 . 0 0 4 8}$ & $\pm \mathbf{0 . 0 0 1 5}$ & {$[81]^{*}$} & $\mathrm{O}\left(\alpha_{s}^{2}\right)+\mathrm{LL}+\mathrm{NLL}$ \\
$\mathrm{T}$ & 0.118 & $\pm_{0.007}^{0.009}$ & $\pm \mathbf{0 . 0 0 4}$ & {$[82]$} & $\mathrm{O}\left(\alpha_{s}^{2}\right)+\mathrm{LL}+\mathrm{NLL}$ \\
$M_{\mathrm{H}, \mathrm{T}}$ & 0.124 & $\pm_{0.006}^{0.007}$ & $\pm \mathbf{0 . 0 0 3}$ & {$[82]$} & $\mathrm{O}\left(\alpha_{s}^{2}\right)+\mathrm{LL}+\mathrm{NLL}$ \\
$\mathrm{EEC}$ & 0.134 & $\pm_{0.006}^{0.006}$ & $\pm \mathbf{0 . 0 0 3}$ & {$[82]$} & $\mathrm{O}\left(\alpha_{s}^{2}\right)+\mathrm{LL}+\mathrm{NLL}$ \\
$\left\langle n_{\text {jet }}\right\rangle$ & 0.132 & $\pm_{0.005}^{0.007}$ & $\pm \mathbf{0 . 0 0 3}$ & {$[82]$} & $\mathrm{O}\left(\alpha_{s}^{2}\right)+\mathrm{LL}+\mathrm{NLL}$ \\
$\mathrm{EEC}$ & 0.121 & $\pm_{0.010}^{0.012}$ & $\pm \mathbf{0 . 0 0 4}$ & {$[232]^{*}$} & $\mathrm{O}\left(\alpha_{s}^{2}\right)$ \\
$\mathrm{AEEC}$ & 0.115 & $\pm_{\mathbf{0 . 0 0 9}}^{0.007}$ & $\pm \mathbf{0 . 0 0 4}$ & {$[232]^{*}$} & $\mathrm{O}\left(\alpha_{s}^{2}\right)$ \\
$R_{3}(\mathrm{~J}, \mathrm{E})$ & 0.115 & $\pm_{\mathbf{0 . 0 1 3}}^{0.013}$ & $\pm \mathbf{0 . 0 0 5}$ & {$[233]^{*}$} & $\mathrm{O}\left(\alpha_{s}^{2}\right)$ \\
\hline
\end{tabular}

Table 20: Compilation of individual $\alpha_{s}$-measurements performed by the L3 collaboration. 


\begin{tabular}{|c|c|c|c|c|c|}
\hline Observable & $\alpha_{s}\left(M_{Z}\right)$ & Total Error & Exp. Error & Reference & Theory \\
\hline$\overline{D_{2}(E)}$ & 0.119 & \pm 0.008 & \pm 0.002 & {$[59]^{*}$} & $\mathrm{O}\left(\alpha_{s}^{2}\right)$ \\
\hline$D_{2}(R)$ & 0.116 & \pm 0.008 & \pm 0.003 & {$[59]^{*}$} & $\mathrm{O}\left(\alpha_{s}^{2}\right)$ \\
\hline $\mathrm{T}$ & 0.121 & $\pm_{0.008}^{0.009}$ & \pm 0.003 & {$[73]^{*}$} & $\mathrm{O}\left(\alpha_{s}^{2}\right)+\mathrm{LL}+\mathrm{NLL}$ \\
\hline$M_{\mathrm{H}, \mathrm{T}}$ & 0.119 & $\pm_{0.007}^{0.008}$ & \pm 0.002 & {$[73]^{*}$} & $\mathrm{O}\left(\alpha_{s}^{2}\right)+\mathrm{LL}+\mathrm{NLL}$ \\
\hline EEC & 0.132 & $\pm_{0.007}^{0.008}$ & \pm 0.003 & {$[73]^{*}$} & $\mathrm{O}\left(\alpha_{s}^{2}\right)+\mathrm{LL}+\mathrm{NLL}$ \\
\hline$y_{3}(\mathrm{D}, \mathrm{E})$ & 0.122 & $\pm_{0.007}^{0.007}$ & \pm 0.004 & {$[73]^{*}$} & $\mathrm{O}\left(\alpha_{s}^{2}\right)+\mathrm{LL}+\mathrm{NLL}$ \\
\hline$B_{t o t}$ & 0.120 & $\pm_{0.005}^{0.006}$ & \pm 0.003 & {$[73]^{*}$} & $\mathrm{O}\left(\alpha_{s}^{2}\right)+\mathrm{LL}+\mathrm{NLL}$ \\
\hline$B_{w}$ & 0.110 & $\pm_{0.005}^{0.006}$ & \pm 0.003 & {$[73]^{*}$} & $\mathrm{O}\left(\alpha_{s}^{2}\right)+\mathrm{LL}+\mathrm{NLL}$ \\
\hline$\left\langle n_{\text {jet }}\right\rangle$ & 0.123 & $\pm_{0.008}^{0.009}$ & \pm 0.003 & {$[73]^{*}$} & $\mathrm{O}\left(\alpha_{s}^{2}\right)+\mathrm{LL}+\mathrm{NLL}$ \\
\hline $\mathrm{T}$ & 0.123 & \pm 0.013 & & {$[73]$} & $\mathrm{O}\left(\alpha_{s}^{2}\right)$ \\
\hline$M_{\mathrm{H}, \mathrm{T}}$ & 0.123 & \pm 0.007 & & {$[73]$} & $\mathrm{O}\left(\alpha_{s}^{2}\right)$ \\
\hline$B_{t o t}$ & 0.136 & \pm 0.015 & & {$[73]^{*}$} & $\mathrm{O}\left(\alpha_{s}^{2}\right)$ \\
\hline$B_{w}$ & 0.122 & \pm 0.007 & & {$[73]^{*}$} & $\mathrm{O}\left(\alpha_{s}^{2}\right)$ \\
\hline EEC & 0.120 & \pm 0.009 & & {$[73]$} & $\mathrm{O}\left(\alpha_{s}^{2}\right)$ \\
\hline$y_{3}(\mathrm{D}, \mathrm{E})$ & 0.119 & \pm 0.010 & & {$[73]$} & $\mathrm{O}\left(\alpha_{s}^{2}\right)$ \\
\hline$\left\langle n_{\text {jet }}\right\rangle$ & 0.122 & \pm 0.012 & & {$[73]^{*}$} & $\mathrm{O}\left(\alpha_{s}^{2}\right)$ \\
\hline$C^{J}$ & 0.128 & $\pm_{0.016}^{0.016}$ & \pm 0.002 & {$[74]^{*}$} & $\mathrm{O}\left(\alpha_{s}^{2}\right)$ \\
\hline 0 & 0.122 & $\pm_{0.008}^{0.047}$ & \pm 0.002 & {$[74]^{*}$} & $\mathrm{O}\left(\alpha_{s}^{2}\right)$ \\
\hline $\mathrm{T}$ & 0.129 & $\pm_{0.017}^{0.017}$ & \pm 0.003 & {$[74]^{*}$} & $\mathrm{O}\left(\alpha_{s}^{2}\right)$ \\
\hline$M_{\mathrm{H}, \mathrm{T}}$ & 0.129 & $\pm_{0.010}^{0.010}$ & \pm 0.002 & {$[74]^{*}$} & $\mathrm{O}\left(\alpha_{s}^{2}\right)$ \\
\hline$M_{\mathrm{D}, \mathrm{T}}$ & 0.120 & $\pm_{0.009}^{0.013}$ & $\pm_{0.004}^{0.001}$ & {$[74]^{*}$} & $\mathrm{O}\left(\alpha_{s}^{2}\right)$ \\
\hline$M_{\mathrm{H}, \mathrm{m}}$ & 0.128 & $\pm_{0.010}^{0.010}$ & $\pm_{0.002}^{0.001}$ & {$[74]^{*}$} & $\mathrm{O}\left(\alpha_{s}^{2}\right)$ \\
\hline$M_{\mathrm{D}, \mathrm{m}}$ & 0.119 & $\pm_{0.007}^{0.012}$ & \pm 0.002 & {$[74]^{*}$} & $\mathrm{O}\left(\alpha_{s}^{2}\right)$ \\
\hline$y_{3}(\mathrm{D}, \mathrm{E})$ & 0.125 & $\pm_{0.007}^{0.008}$ & \pm 0.004 & {$[74]^{*}$} & $\mathrm{O}\left(\alpha_{s}^{2}\right)$ \\
\hline$y_{3}(\mathrm{M}, \mathrm{E} 0)$ & 0.120 & $\pm_{0.008}^{0.009}$ & \pm 0.002 & {$[74]^{*}$} & $\mathrm{O}\left(\alpha_{s}^{2}\right)$ \\
\hline$y_{3}(\mathrm{M}, \mathrm{E})$ & 0.128 & $\pm_{0.016}^{0.016}$ & \pm 0.003 & {$[74]^{*}$} & $\mathrm{O}\left(\alpha_{s}^{2}\right)$ \\
\hline$y_{3}(\mathrm{M}, \mathrm{P})$ & 0.122 & $\pm_{0.004}^{0.009}$ & \pm 0.002 & {$[74]^{*}$} & $\mathrm{O}\left(\alpha_{s}^{2}\right)$ \\
\hline AEEC & 0.118 & $\pm_{0.005}^{0.009}$ & \pm 0.003 & {$[74]^{*}$} & $\mathrm{O}\left(\alpha_{s}^{2}\right)$ \\
\hline PTEC & 0.112 & $\pm_{0.010}^{0.010}$ & \pm 0.009 & {$[74]^{*}$} & $\mathrm{O}\left(\alpha_{s}^{2}\right)$ \\
\hline$M_{\mathrm{H}, \mathrm{T}}$ & 0.117 & $\pm_{0.005}^{0.005}$ & & {$[74]$} & $\mathrm{O}\left(\alpha_{s}^{2}\right)+\mathrm{LL}+\mathrm{NLL}$ \\
\hline$M_{\mathrm{H}, \mathrm{m}}$ & 0.117 & $\pm_{0.006}^{0.006}$ & & {$[74]$} & $\mathrm{O}\left(\alpha_{s}^{2}\right)+\mathrm{LL}+\mathrm{NLL}$ \\
\hline $\mathrm{T}$ & 0.127 & $\pm_{0.007}^{0.002}$ & & {$[74]$} & $\mathrm{O}\left(\alpha_{s}^{2}\right)+\mathrm{LL}+\mathrm{NLL}$ \\
\hline AEEC & 0.118 & $\pm_{0.005}^{0.010}$ & \pm 0.003 & [234] & $\mathrm{O}\left(\alpha_{s}^{2}\right)$ \\
\hline$y_{3}(\mathrm{~J}, \mathrm{E})$ & 0.118 & \pm 0.009 & \pm 0.003 & {$[75]$} & $\mathrm{O}\left(\alpha_{s}^{2}\right)$ \\
\hline$y_{3}(\mathrm{M}, \mathrm{E})$ & 0.126 & \pm 0.014 & \pm 0.003 & {$[75]$} & $\mathrm{O}\left(\alpha_{s}^{2}\right)$ \\
\hline$y_{3}(\mathrm{M}, \mathrm{P} 0)$ & 0.118 & \pm 0.008 & \pm 0.003 & {$[75]^{*}$} & $\mathrm{O}\left(\alpha_{s}^{2}\right)$ \\
\hline$y_{3}(\mathrm{M}, \mathrm{P})$ & 0.118 & \pm 0.008 & \pm 0.003 & {$[75]$} & $\mathrm{O}\left(\alpha_{s}^{2}\right)$ \\
\hline EEC & 0.131 & \pm 0.009 & \pm 0.006 & [235] & $\mathrm{O}\left(\alpha_{s}^{2}\right)$ \\
\hline AEEC & 0.117 & \pm 0.009 & $\pm_{0.009}^{0.007}$ & {$[235]$} & $\mathrm{O}\left(\alpha_{s}^{2}\right)$ \\
\hline EEC & 0.117 & $\pm_{0.008}^{0.006}$ & & [235] & $\mathrm{O}\left(\alpha_{s}^{2}\right)$ \\
\hline$y_{3}(\mathrm{~J}, \mathrm{E})$ & 0.117 & \pm 0.015 & & [236] & $\mathrm{O}\left(\alpha_{s}^{2}\right)$ \\
\hline
\end{tabular}

Table 21: Compilation of individual $\alpha_{s}$-measurements done by the OPAL collaboration. The numbers for reference $[236]$ correspond to $80 \mathrm{MeV} \leq \Lambda \frac{(5)}{\mathrm{MS}} \leq 450 \mathrm{MeV}$. 


\section{References}

[1] M.Y. Han and Y. Nambu, Phys. Rev. B139 (1965) 1006.

[2] L. Montanet et al., Phys. Rev. D50 (1994) 1449.

[3] L. Montanet et al., Phys. Rev. D50 (1994) 1334.

[4] H. Fritzsch, M. Gell-Mann and H. Leutwyler, Phys. Lett. B47 (1973) 365.

[5] D.J. Gross and F. Wilczek, Phys. Rev. Lett. 30 (1973) 1343.

[6] H.D. Politzer, Phys. Rev. Lett. 30 (1973) 1346.

[7] ALEPH collaboration, D. Buskulic et al., Phys. Lett. B303 (1993) 198.

[8] B. Bambha et al., "QCD Generators for LEP", Preprint CERN-TH.5466/89.

[9] T. Sjöstrand, Comp. Phys. Comm. 39 (1986) 347.

[10] R. Odorico, Nucl. Phys. B228 (1983) 381.

[11] R. Odorico, Comp. Phys. Comm. 32 (1984) 139, Erratum Comp. Phys. Comm. 34 (1985) 43.

[12] R. Mazzanti and R. Odorico, Nucl. Phys. B370 (1992) 23.

[13] R. Mazzanti and R. Odorico, Bologna Preprint DFUB 92/1.

[14] T. Sjöstrand and M. Bengtson, Comp. Phys. Comm. 43 (1987) 367.

[15] M. Bengtson and T. Sjöstrand, Phys. Lett. B185 (1987) 435.

[16] M. Bengtson and T. Sjöstrand, Nucl. Phys. B289 (1987) 810.

[17] G. Marchesini and B.R. Webber, Nucl. Phys. B310 (1988) 461.

[18] I.G. Knowles, Nucl. Phys. B310 (1988) 571.

[19] G. Marchesini et al., Comp. Phys. Comm. 67 (1992) 465.

[20] K. Kato and T. Munehisa, Phys. Rev. D36 (1987) 61.

[21] K. Kato and T. Munehisa, Comp. Phys. Comm. 64 (1991) 67.

[22] U. Petterson, LU TP 88-5.

[23] L. Lönnblad and U. Petterson, LU TP 88-15.

[24] L. Lönnblad, LU TP 91-11.

[25] B. Anderson, G. Gustavson and C. Peterson, Z. Phys. C1 (1979) 105. 
[26] B. Anderson, G. Gustavson and B. Söderberg, Z. Phys. C20 (1983) 317.

[27] C. Peterson et al., Phys. Rev. D27 (1983) 105.

[28] L3 Collaboration, B. Adeva et al., Z. Phys. C55 (1992) 39.

[29] ALEPH Collaboration, D. Buskulic et al., Z. Phys. C55 (1992) 209.

[30] E. Fahri, Phys. Rev. Lett. 39 (1977) 1587.

[31] R.F. Schwitters et al., Phys. Rev. Lett. 35 (1975) 1320.

[32] G. Hanson et al., Phys. Rev. Lett. 35 (1975) 1609.

[33] TASSO Collaboration, R. Brandelik et al., Phys. Lett. B97 (1980) 453.

[34] PLUTO Collaboration, C. Berger et al., Phys. Lett. B97 (1980) 459.

[35] CELLO Collaboration, H.-J. Behrend et al., Phys. Lett. B110 (1982) 329.

[36] S.L. Wu, Phys. Rep. 107 (1984) 59.

[37] ALEPH Collaboration, contributed Paper to ICHEP94, Glasgow, 20-27 July 1994, Ref. 0529.

[38] L3 Collaboration, B. Adeva et al., Phys. Lett. B264 (1991) 551.

[39] OPAL Collaboration, G. Alexander et al., Z. Phys. C52 (1991) 543.

[40] DELPHI Collaboration, P. Abreu et al., Phys. Lett. B274 (1992) 498.

[41] E. Laermann, K.H. Streng and P.M. Zerwas, Z. Phys. C3 (1980) 289 and Erratum Z. Phys. C52 (1991) 352.

[42] J. Ellis and I. Karliner, Nucl. Phys. B148 (1979) 141.

[43] J.G. Körner, G.A. Schuler and F. Barreiro, Phys. Lett. B188 (1987) 272.

[44] G.A. Schuler and J.G. Körner, Nucl. Phys. B325 (1989) 557.

[45] W. Bernreuther and W. Wetzel, Nucl. Phys. B197 (1982) 228.

[46] G. Rodrigo and A. Santamaria, "QCD matching conditions at thresholds", CERNTH.6899/93.

[47] T. Hebbeker, M. Martinez, G. Passarino and G. Quast, CERN-PPE/94-44.

[48] CDF Collaboration, F. Abe et al., "Evidence for Top quark production in $p \bar{p}$ collisions at $\sqrt{s}=1.8 \mathrm{TeV}$ ", to be published in Phys. Rev. D.

[49] A. Blondel, CERN-PPE/94-133.

[50] MARK-J Collaboration, D.P. Barber et al., Phys. Rev. D43 (1979) 830. 
[51] T. Chandrahoman and L. Clavelli, Nucl. Phys. B184 (1981) 365.

[52] S. Catani, G. Turnock and B.R. Webber, CERN-TH.6570/92

[53] G. Parisi, Phys. Lett. B74 (1978) 65.

[54] R.K. Ellis, D.A. Ross and A.E. Terrano, Nucl. Phys. B178 (1981) 421.

[55] C. Basham, L. Brown, S. Ellis and S. Love, Phys. Rev. Lett. 41 (1978) 1585.

[56] F. Csikor, G. Pócik and A. Tóth, Phys. Rev. D31 (1985) 1025.

[57] G. Sterman and S. Weinberg, Phys. Rev. Lett. 39 (1977) 1436.

[58] S.D. Ellis, Z. Kunszt and D.E. Soper, Phys. Rev. 62 (1989) 726.

[59] OPAL Collaboration, R. Akers et al., CERN-PPE/94-51.

[60] S. Bethke, Z. Kunszt, D.E. Soper and W.J. Stirling, Nucl. Phys. B370 (1992) 310.

[61] S. Catani, Yu.L. Dokshitzer, F. Fiorani and B.R. Webber, Nucl. Phys. B377 (1992) 445.

[62] ALEPH Collaboration, D. Decamp et al., Phys. Lett. B257 (1991) 479.

[63] F. Bloch and A. Nordsieck, Phys. Rev. 52 (1937) 54.

[64] T. Kinoshita, J. Math. Phys. 3 (1962) 650.

[65] T.D. Lee and M. Nauenberg, Phys. Rev. 133 (1964) 1549.

[66] S. Catani, L. Trentadue, G. Turnock and B.R. Webber, CERN-TH.6640/92 and CavendishHEP-91/11.

[67] P. Nason, Program EVENT - Monte-Carlo integration of ERT matrix elements

[68] ALEPH Collaboration, D. Buskulic et al., Phys. Lett. B307 (1993) 209.

[69] M. Schmelling and R.D. St.Denis, Phys. Lett. B329 (1994) 393 and preprint CERN/PPE 93-193 and MPI-PhE/93-31.

[70] M. Kobel, "Determination of $\alpha_{s}$ and $\Lambda$ from heavy quarkonium decays", Proc. XXVIIth Rencontres de Moriond, Les Arcs, 22-28 March 1992.

[71] DELPHI Collaboration, P. Abreu et al., Z. Phys. C54 (1992) 55.

[72] L3 Collaboration, O. Adriani et al., Phys. Rep. 236 (1993) 1.

[73] OPAL Collaboration, P.D. Acton et al., CERN-PPE/93-38.

[74] OPAL Collaboration, P.D. Acton et al., Z. Phys. C55 (1992) 1.

[75] OPAL Collaboration, M.Z. Akrawy et al., CERN-PPE/90-143 + Erratum. 
[76] Mark II Collaboration, S. Komamiya et al., Phys. Rev. Lett. 64 (1990) 987.

[77] SLD Collaboration, K. Abe et al., SLAC-PUB-6133, July 1993.

[78] ALEPH Collaboration, D. Decamp et al., Phys. Lett. B284 (1992) 163.

[79] ALEPH Collaboration, ALEPH note 92-114, PHYSIC 92-103, Dallas 1992.

[80] DELPHI Collaboration, P. Abreu et al., CERN-PPE/93-43 subm. to ZPC.

[81] L3 Collaboration, L3 note \#1441, Marseille 1993.

[82] L3 Collaboration, O. Adriani et al., Phys. Lett. B284 (1992) 471.

[83] M. Schmelling, "Averaging Correlated Data", to appear in the same issue.

[84] P. Nason and B.R. Webber, CERN-TH.70118/93.

[85] G. Gurci, W. Furmanski and R. Petronzio, Nucl. Phys. B175 (1980) 27.

[86] W. Furmanski and R. Petronzio, CERN-TH.2933 and Nucl. Phys. B175 (1980) 27.

[87] G. Altarelli, R.K. Ellis, G. Martinelli and So-Young Pi, Nucl. Phys. B160 (1979) 301.

[88] M. Virchaux and A. Milsztajn, Phys. Lett. B274 (1992) 221.

[89] DELPHI Collaboration, P. Abreu et al., Phys. Lett. B311 (1993) 408.

[90] ALEPH Collaboration, contributed Paper to ICHEP94, Glasgow, 20-27 July 1994, Ref. 0525.

[91] M.A. Shifman, A.L. Vainshtein and V.I. Zakharov, Nucl. Phys. B147 (1979) 385, 448, 519.

[92] E. Braaten, S. Narison and A. Pich, Nucl. Phys. B373 (1992) 581.

[93] F. Le Diberder and A. Pich, Phys. Lett. B286 (1992)147.

[94] F. Le Diberder and A. Pich, Phys. Lett. B289 (1992)165.

[95] A. Pich, "QCD predictions for the tau hadronic width and determination of $\alpha_{s}\left(M_{\tau}\right)$ ", CERNTH.6738/92.

[96] ALEPH Collaboration, L. Duflot et al., " $\alpha_{s}$ from spectral moments", Talk given at the 3rd Workshop on Tau Lepton Physics, Montreux, 19-22 September 1994.

[97] CLEO Collaboration, D. Miller et al., contributed Paper to ICHEP94, Glasgow, 20-27 July 1994, ref GLS0383.

[98] R. Patterson, Plenary talk "Weak Rare Decays", ICHEP94, Glasgow, 20-27 July 1994.

[99] A. Kounine, XXIX Rencontres de Moriond, Meribel, France, March 12-19, 1994.

[100] E. Fernandez, “Tau Physics”, IFAE 93-001, December 20, 1993. 
[101] S. Bethke and S. Catani, "A summary of $\alpha_{s}$ measurements", Proc. XXVIIth Rencontres de Moriond, Les Arcs, 22-28 March 1992, and preprint CERN-TH/6484-92.

[102] G. Altarelli, "QCD and experiment: Status of $\alpha_{s}$ ", Proc. QCD 20 years later, Aachen, 9-13 June 1992, and preprint CERN-TH/6623-92.

[103] BCDMS Collaboration, A.C. Benvenuti et al., Phys. Lett. B195 (1987) 97.

[104] S. Wimpenny, EMC Collaboration, Proc. Lepton-Photon Symposium, Geneva, 21 July - 1 August, 1991, and preprint UCR DIS 91-03.

[105] NMC Collaboration, M. Arneodo et al., Phys. Lett. B309 (1993) 222.

[106] CHARM Collaboration, F. Bergsma et al., Phys. Lett. B153 (1985) 111.

[107] CDHSW Collaboration, P. Berge et al., Z. Phys. C49 (1990) 187.

[108] CCFR Collaboration, W.H. Smith et al., "QCD analysis of neutrino scattering results from CCFR", WISC EX 93-331.

[109] M. Virchaux, "Nucleon structure functions", Proc. QCD 20 years later, Aachen, 9-13 June 1992, and preprint DAPNIA SPP 92-30.

[110] G. d'Agostini, W. de Boer and G. Grindhammer, Phys. Lett. B229 (1989) 160.

[111] R. Marshall, Z. Phys. C43 (1989) 595.

[112] The LEP Electroweak Working Group,CERN-PPE/93-157.

[113] S.G. Gorishny, A.L. Kataev and S.A. Larin, Phys. Lett. B212 (1988) 238.

[114] S.G. Gorishny, A.L. Kataev and S.A. Larin, Phys. Lett. B259 (1991) 144.

[115] L.R. Surguladze and M.A. Samuel, Phys. Rev. Lett. 66 (1991) 560.

[116] CLEO Collaboration, D. Miller et al., contributed Paper to ICHEP94, Glasgow, 20-27 July 1994, Ref. 0168.

[117] S. Bethke, "Recent results on jet physics and tests of QCD in $e^{+} e^{-}$annihilation", preprint LBL-28112, November 1989.

[118] B. Naroska, Phys. Rep. 148 (1987) 67.

[119] S.L. Wu, Proc. Lepton-Photon Symposium, Hamburg, July 27-31, 1987.

[120] VENUS Collaboration, K. Abe et al., Phys. Lett. B240 (1990) 232.

[121] AMY Collaboration, K.B. Lee et al., Phys. Lett. B313 (1993) 469.

[122] TOPAZ Collaboration, Y. Ohnishi et al., Phys. Lett. B313 (1993) 475.

[123] A.X. El-Khadra, G. Hockney, A.S. Kronfeld and P.B. Mackenzie, Phys. Rev. Lett. 69 (1992) 729. 
[124] A.X. El-Khadra, "The strong coupling from Quarkonia", Proc. Lattice '93, Dallas, October $12-16,1993$.

[125] P. Lepage and J. Sloan, "Precision Upsilon spectroscopy and fundamental parameters from NRQCD", Proc. Lattice '93, Dallas, October 12-16, 1993.

[126] C.T.H Davies, K.Hornbostel, G.P. Lepage, A. Lidsey, J. Shigemitsu and J. Sloan, “A Precise Determination of $\alpha_{s}$ From Lattice QCD", contributed Paper to ICHEP94, Glasgow, 20-27 July 1994, HEP-PH-9408328.

[127] J. Shigemitsu, Plenary talk "Latice Gauge Theory", ICHEP94, Glasgow, 20-27 July 1994.

[128] A.Geiser (UA1 Collaboration), PITHA 92/19 (1992).

[129] UA2 Collaboration, J. Alitti et al., Phys. Lett. B263 (1991) 563.

[130] UA6 Collaboration, G. Ballocchi et al., CERN-PPE/91-129.

[131] T.N. Truong, "Uncertainties in the determination of $\alpha_{s}$ using hadronic $\tau$ decay data and $e^{+} e^{-}$hadronic cross sections", preprint, EP-CPth.A266.1093, January 1994.

[132] G. Altarelli, " $\alpha_{s}$ from tau", Talk given at the 3rd Workshop on Tau Lepton Physics, Montreux, 19-22 September 1994.

[133] B.L. Ioffe, Phys. Lett. B78 (1978) 277.

[134] E. Laermann and P.M. Zerwas, Phys. Lett. B89 (1980) 225.

[135] A. Ballestrero, E. Maina and S. Moretti, Phys. Lett. B294 (1992) 425.

[136] A. Ballestrero, E. Maina and S. Moretti, Nucl. Phys. B415 (1994) 265.

[137] ALEPH Collaboration, Internal note 93-147, PHYSIC 93-127.

[138] ALEPH Collaboration, contributed Paper to ICHEP94, Glasgow, 20-27 July 1994, Ref. 0527.

[139] DELPHI Collaboration, P. Abreu et al., CERN-PPE/93-59.

[140] L3 Collaboration, B. Adeva et al., Phys. Lett. B271 (1991) 461.

[141] OPAL Collaboration, R. Akers et al., Z. Phys. C60 (1993) 397.

[142] OPAL Collaboration, R. Akers et al., CERN-PPE/94-123.

[143] M. Hildreth, SLD Collaboration, XXIX Rencontres de Moriond, Meribel, France, March 19-26, 1994.

[144] O. Biebel and P. Mättig, private communication.

[145] ALEPH Collaboration, D. Decamp et al., Phys. Lett. B284 (1992) 151.

[146] A. Ricker, L3 Collaboration, XXIX Rencontres de Moriond, Meribel, France, March 19-26, 1994. 
[147] L3 Collaboration, contributed Paper to ICHEP94, Glasgow, 20-27 July 1994, Ref. 0630.

[148] J.G. Körner, G. Schierholz and J. Willrodt, Nucl. Phys. B185 (1981) 365.

[149] O. Nachtmann and A. Reiter, Z. Phys. C16 (1982) 45.

[150] M. Bengtson and P.M. Zerwas, Phys. Lett. B208 (1988) 306.

[151] M. Bengtson, Z. Phys. C42 (1989) 75.

[152] G. Rudolph, "Physics at LEP", CERN 86-02 (1986) Vol.2, 150.

[153] AMY Collaboration, I.H. Park et al., Phys. Rev. Lett. 62 (1989) 1713.

[154] VENUS Collaboration, K. Abe et al., Phys. Rev. Lett. 66 (1991) 280.

[155] ABCDHW Collaboration, A. Breakstone et al., Phys. Lett. B248 (1990) 220.

[156] L3 Collaboration, B. Adeva et al., Phys. Lett. B248 (1990) 227.

[157] OPAL Collaboration, M.Z. Akrawy et al., Z. Phys. C49 (1991) 49.

[158] DELPHI Collaboration, P. Abreu et al., Phys. Lett. B255 (1991) 466.

[159] DELPHI Collaboration, P. Abreu et al., Z. Phys. C59 (1993) 357.

[160] DELPHI Collaboration, contributed Paper to ICHEP94, Glasgow, 20-27 July 1994, Ref. 0180 and A. Seitz, private communication.

[161] OPAL Collaboration, contributed Paper to ICHEP94, Glasgow, 20-27 July 1994, Ref. 0597.

[162] ALEPH-Collaboration, contributed Paper to ICHEP94, Glasgow, 20-27 July 1994, Ref. 0546.

[163] Achim Geiser, "Measurement of the triple-gluon vertex from jet and heavy flavour production at the CERN $p \bar{p}$ collider", CERN-PPE/94-38.

[164] OPAL Collaboration, contributed Paper to ICHEP94, Glasgow, 20-27 July 1994, Ref. 0601 and S. Kluth, private communication.

[165] Ya.I. Azimov, Yu.L. Dokshitzer, V.A. Khoze and S.I.Troyan, Z. Phys. C31 (1986) 213.

[166] C.P. Fong and B.R. Webber, Nucl. Phys. B335 (1991) 54.

[167] Ya.I. Azimov, Yu.L. Dokshitzer, V.A. Khoze and S.I.Troyan, Z. Phys. C27 (1985) 65.

[168] OPAL Collaboration, M.Z. Akrawy et al., Phys. Lett. B247 (1990) 617.

[169] OPAL Collaboration, R. Akers et al., CERN-PPE/94-49.

[170] L3 Collaboration, M. Acciarri et al., CERN-PPE/94-53.

[171] TASSO Collaboration, W. Braunschweig et al., Z. Phys. C47 (1990) 187. 
[172] MARK II Collaboration, A. Petersen et al., Phys. Rev. D37 (1988) 1.

[173] TPC/2 $\gamma$ Collaboration, H. Aihara et al., LBL-23737 (1988);

update from $\mathrm{G}$. Cowan, private communication.

[174] O. Podobrin, Ph.D. Thesis, University of Hamburg;

see also DELPHI Collaboration, P. Abreu et al., Phys. Lett. B311 (1993) 408.

[175] AMY Collaboration, Y.K. Li et al., Phys. Rev. D411 (1990) 2675.

[176] ALEPH Collaboration, D. Buskulic et al., Z. Phys. C55 (1992) 209.

[177] DELPHI Collaboration, P. Abreu et al., Phys. Lett. B275 (1992) 231.

[178] L3 Collaboration, B. Adeva et al., Phys. Lett. B259 (1991) 199.

[179] ALEPH Collaboration, D. Buskulic et al., CERN-PPE/94-74.

[180] OPAL Collaboration, G. Alexander et al., Phys. Lett. B264 (1991) 467.

[181] L3 Collaboration, O. Adriani et al., Phys. Lett. B286 (1992) 403.

[182] OPAL Collaboration, P.D. Acton et al., Phys. Lett. B291 (1992) 503.

[183] Z. Kunszt, P. Nason, G. Marchesini and B.R. Webber, "Z Physics at LEP", Vol. 1, CERNReport 89-08, p. 434ff.

[184] E.D. Malaza and B.R. Webber, Phys. Lett. B149 (1984) 501.

[185] E.D. Malaza and B.R. Webber, Nucl. Phys. B267 (1986) 702.

[186] JADE Collaboration, W. Bartel et al., Z. Phys. C20 (1983) 187.

[187] PLUTO Collaboration, C. Berger et al., Phys. Lett. B95 (1980) 313.

[188] TASSO Collaboration, W. Braunschweig et al., Z. Phys. C45 (1989) 193.

[189] TPC/2 $\gamma$ Collaboration, H. Aihara et al., Phys. Lett. B134 (1987) 299.

[190] HRS Collaboration, M. Derrick et al., Phys. Rev. D34 (1986) 3304.

[191] TOPAZ Collaboration, M. Yamauchi et al., Proc. XXIVth International Conference on High Energy Physics, Munich, 4-10 August 1988, p. 852.

[192] AMY Collaboration, H.W. Zheng et al., Phys. Rev. D42 (1990) 737.

[193] ALEPH Collaboration, D. Decamp et al., Phys. Lett. B273 (1991) 181.

[194] DELPHI Collaboration, P. Abreu et al., Z. Phys. C50 (1991) 185.

[195] OPAL Collaboration, P.D. Acton et al., Z. Phys. C53 (1992) 539.

[196] L3 Collaboration, B. Adeva et al., CERN-PPE /92-50. 
[197] MARKII Collaboration, G.S. Abrams et al., Phys. Rev. Lett. 64 (1990) 1334.

[198] S. Carius and G. Ingelmann, Phys. Lett. B252 (1990) 647.

[199] R. Szwed and G. Wrochna, Z. Phys. C47 (1990) 447.

[200] A. Bialas and R. Peschanski, Nucl. Phys. B273 (1986) 703.

[201] ALEPH Collaboration, D. Decamp et al., CERN-PPE/91-121.

[202] DELPHI Collaboration, P. Abreu et al., Phys. Lett. B247 (1990) 137.

[203] OPAL Collaboration, M.Z. Akrawy et al., Phys. Lett. B262 (1991) 351.

[204] OPAL Collaboration, P.D. Acton et al., Z. Phys. C54 (1992) 193.

[205] ALEPH Collaboration, D. Buskulic et al., CERN-PPE/92-143.

[206] L3 Collaboration, O. Adriani et al., Phys. Lett. B292 (1992) 472.

[207] S. Catani, F. Fiorani, Yu.L. Dokshitzer and B.R. Webber, Nucl. Phys. B383 (1992) 419.

[208] OPAL Collaboration, R. Akers et al., CERN-PPE/94-52.

[209] DELPHI Collaboration, P. Abreu et al., Phys. Lett. B286 (1992) 201.

[210] DELPHI Collaboration, P. Abreu et al., CERN-PPE/94-03 K0-K0 BE-correlations

[211] OPAL Collaboration, P.D. Acton et al., PLB267 (1991) 143.

[212] OPAL Collaboration, P.D. Acton et al., CERN-PPE/92-192.

[213] ALEPH Collaboration, D. Decamp et al., CERN-PPE/91-183

[214] OPAL Collaboration, P.D. Acton et al., CERN-PPE/93-26.

[215] DELPHI Collaboration, P. Abreu et al., CERN-PPE/93-171.

[216] B. Anderson, G. Gustafson and T. Sjöstrand, Physica Scripta 32 (1985) 574.

[217] S.J. Brodsky and J. Gunion, Phys. Rev. Lett. 37 (1976) 402.

[218] K. Konishi, A. Ukawa and G. Veneziano, Phys. Lett. B78 (1978) 243.

[219] OPAL Collaboration, G. Alexander et al., CERN-PPE/91-91.

[220] OPAL Collaboration, P.D. Acton et al., CERN-PPE/93-02.

[221] DELPHI Collaboration, contributed Paper to ICHEP94, Glasgow, 20-27 July 1994, Ref. 0187.

[222] ALEPH Collaboration, contributed Paper to ICHEP94, Glasgow, 20-27 July 1994, Ref. 0539. 
[223] Ya.I. Azimov, Yu.L. Dokshitzer, V.A. Khoze and S.I. Troyan, Phys. Lett. B165 (1985) 147.

[224] Yu.L. Dokshitzer, V.A. Khoze, S.I. Troyan and A.H. Mueller, Reviews of modern Physics 60 (1988) 373.

[225] OPAL Collaboration, M.Z. Akrawy et al., Phys. Lett. B261 (1991) 334.

[226] ALEPH Collaboration, contributed Paper to ICHEP94, Glasgow, 20-27 July 1994, Ref. 0543.

[227] OPAL Collaboration, M.Z. Akrawy et al., Z. Phys. C47 (1990) 505.

[228] OPAL Collaboration, P.D. Acton et al., Phys. Lett. B276 (1992) 547.

[229] ALEPH Collaboration, D. Decamp et al., Phys. Lett. B255 (1991) 492.

[230] DELPHI Collaboration, P. Abreu et al., Phys. Lett. B252 (1990) 149.

[231] DELPHI Collaboration, P. Abreu et al., Phys. Lett. B247 (1990) 169.

[232] L3 Collaboration, B. Adeva et al., Phys. Lett. B257 (1991) 469.

[233] L3 Collaboration, B. Adeva et al., Phys. Lett. B248 (1990) 464.

[234] OPAL Collaboration, P.D. Acton et al., Phys. Lett. B276 (1992) 547.

[235] OPAL Collaboration, M.Z. Akrawy et al., Phys. Lett. B252 (1990) 159.

[236] OPAL Collaboration, M.Z. Akrawy et al., Phys. Lett. B235 (1990) 389. 


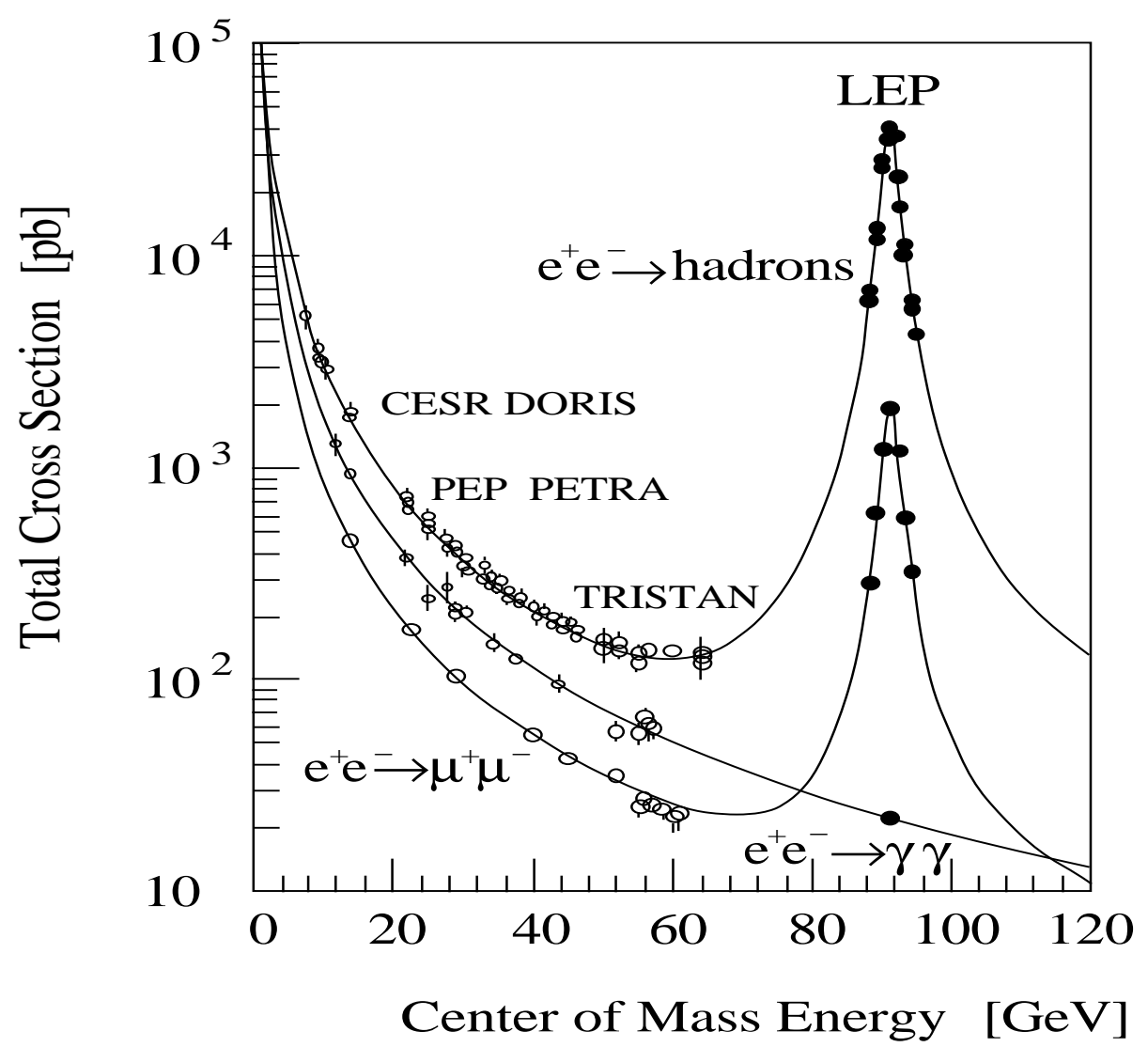

Figure 1: Total cross section for $e^{+} e^{-}$annihilation into hadrons and muon pairs as function of the centre-of-mass energy. Also given is the two-photon cross section. Experimental data are compared with predictions from the standard model.

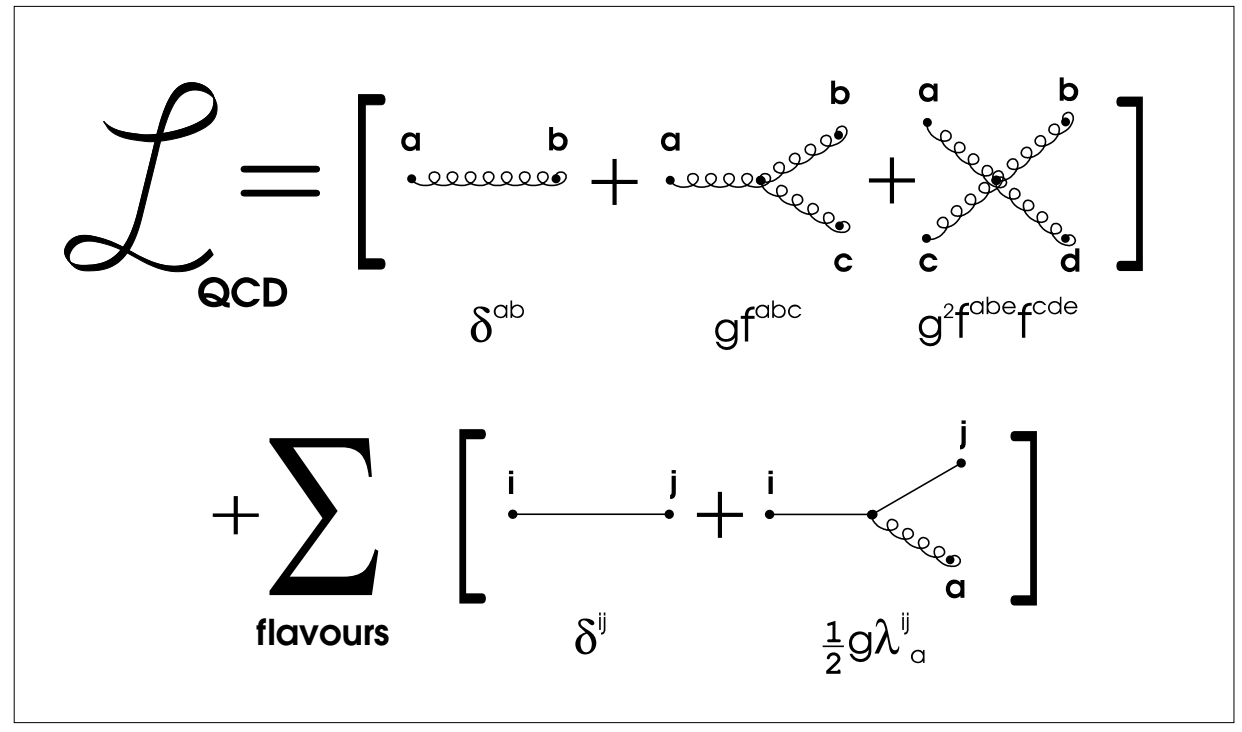

Figure 2: Pictorial representation of the QCD Lagrangian with the factors that determine the coupling strengths. 


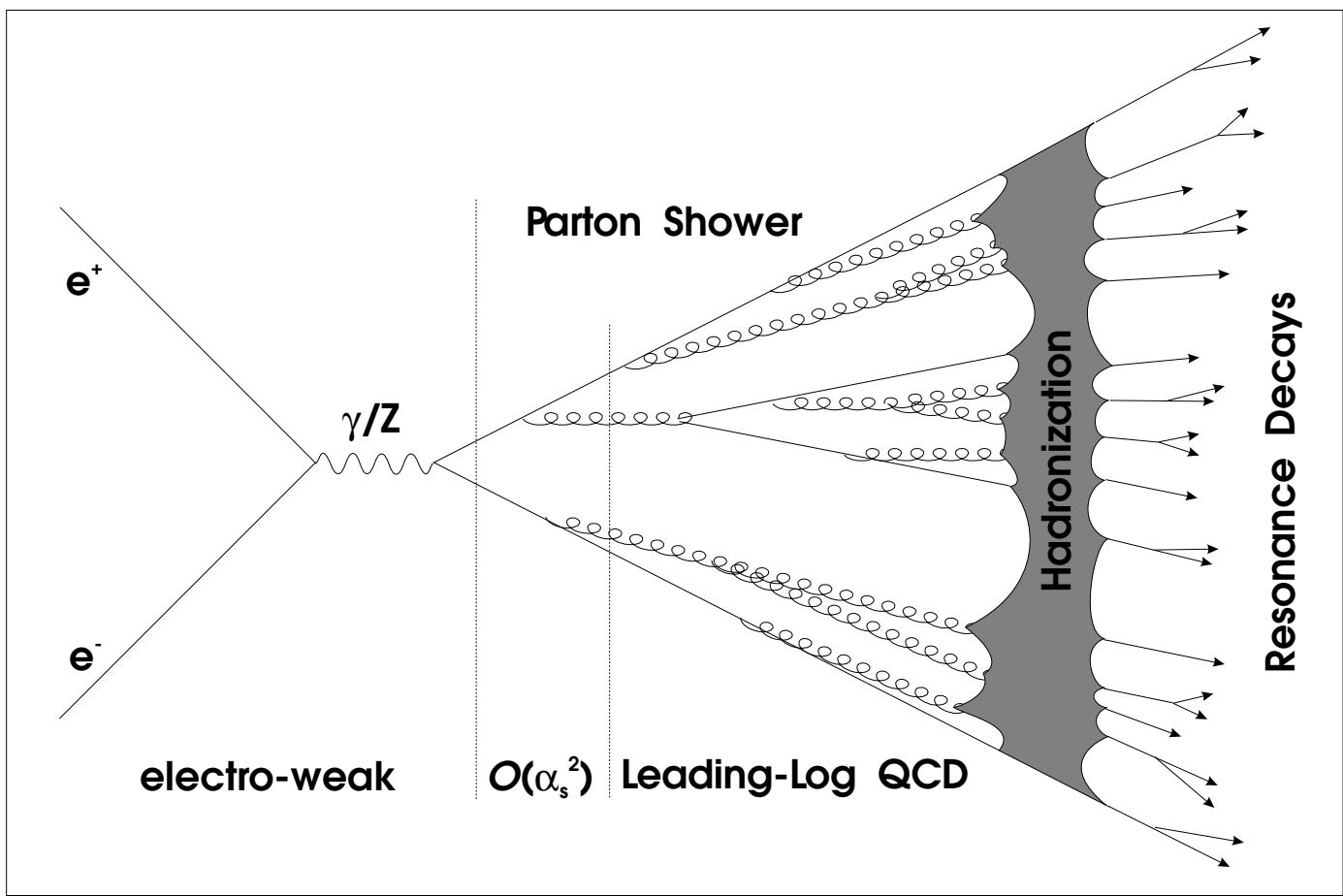

Figure 3: Schematic representation of a parton shower.

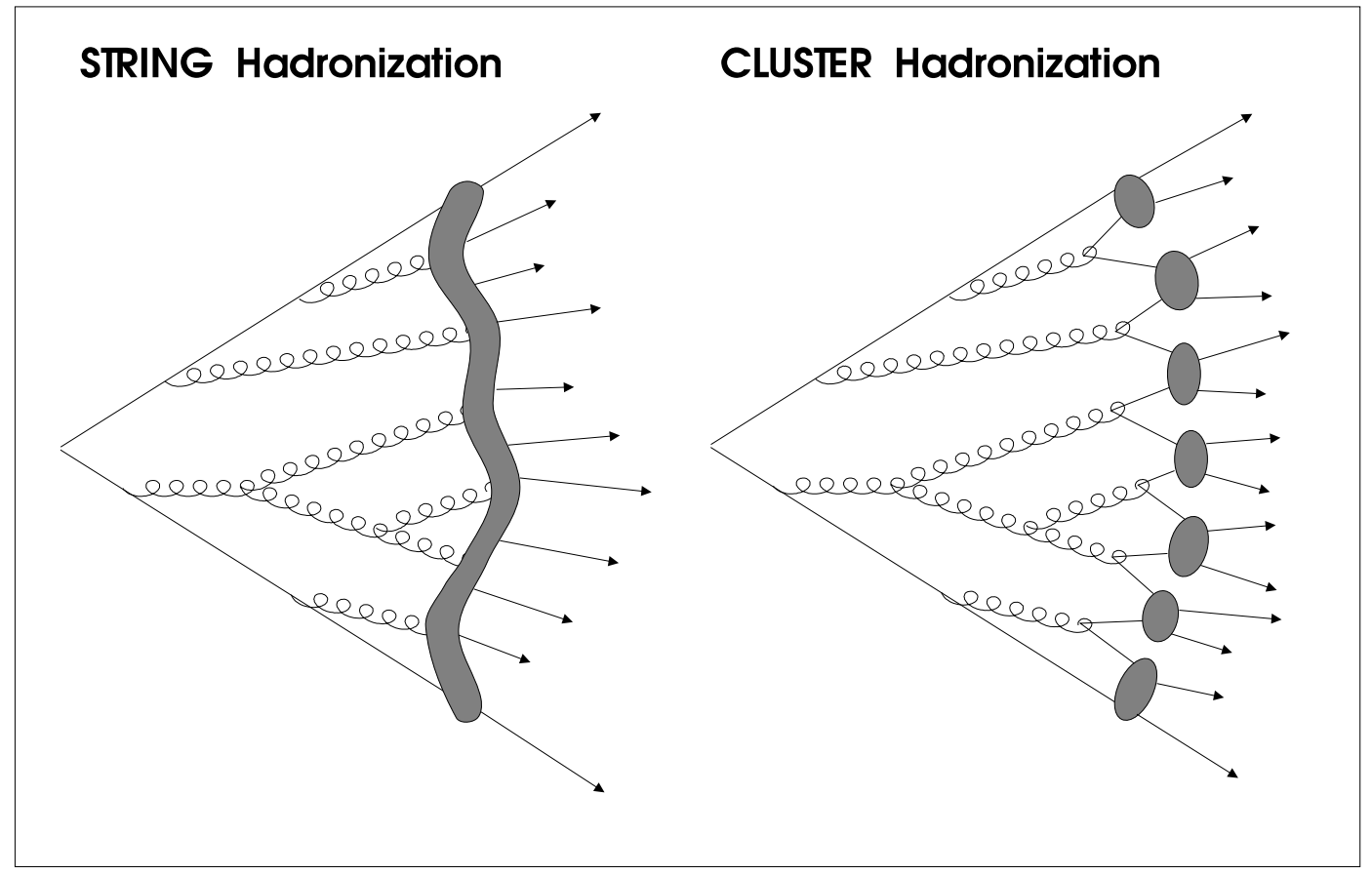

Figure 4: Pictorial presentation of the string and the cluster hadronization model. 


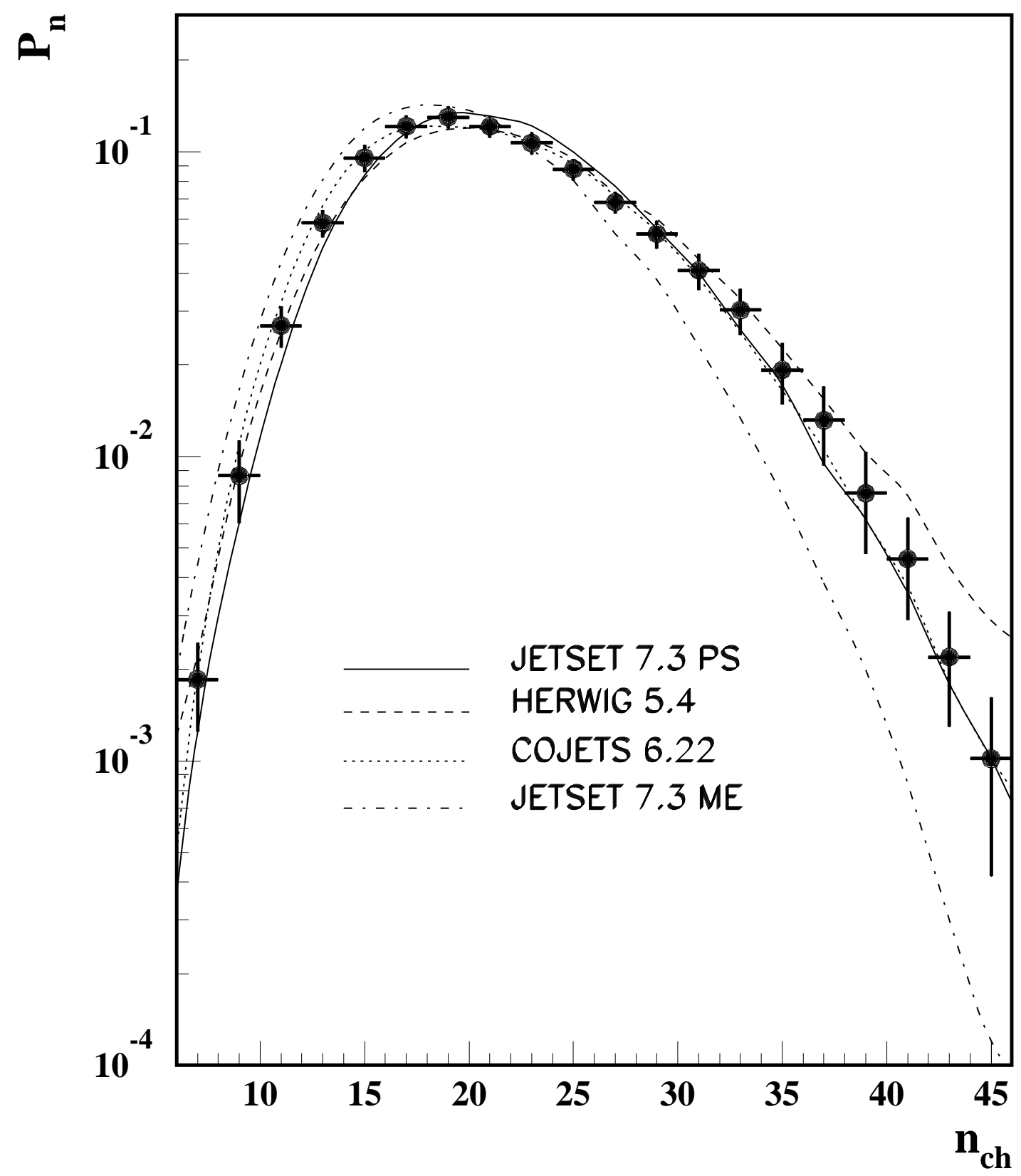

Figure 5: Charged multiplicity distribution measured by the L3 collaboration [28]. The points with error bars are the experimental data, the curves are model predictions. 


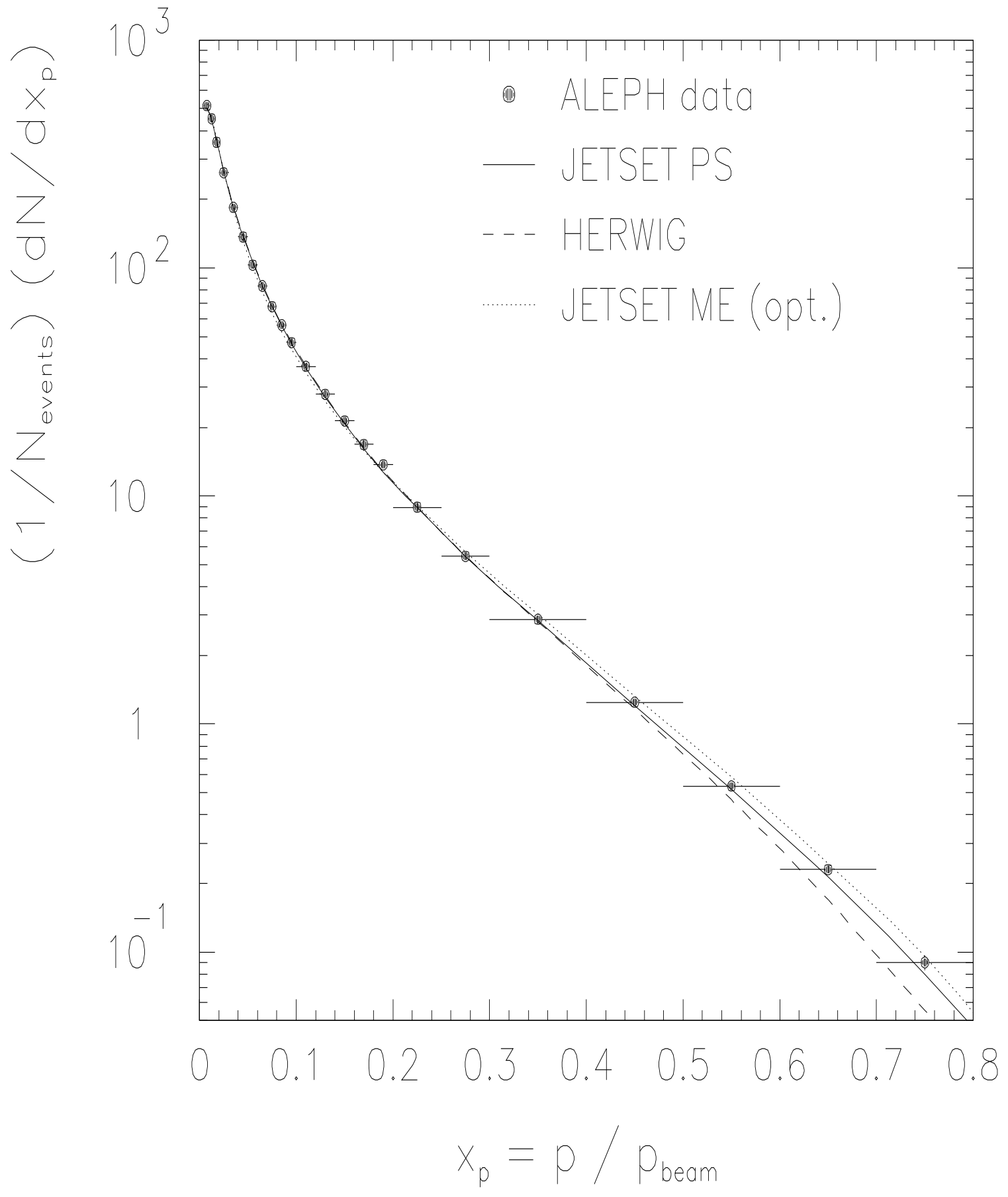

Figure 6: Inclusive momentum distributions $d \sigma / d x$ measured by the ALEPH collaboration [29]. The experimental data are compared to various models. 


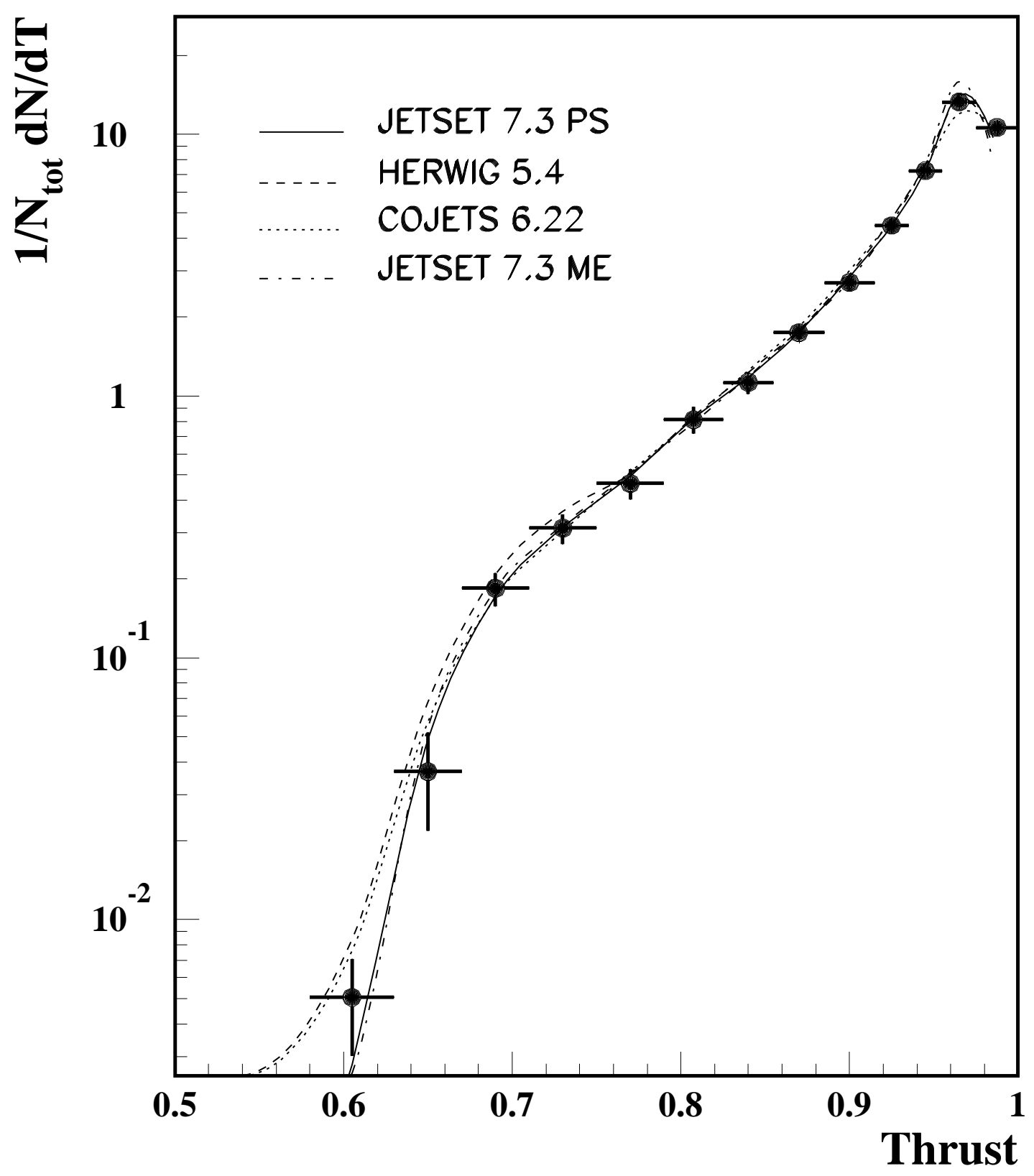

Figure 7: Thrust distribution measured by the L3-collaboration [28]. The points represent the experimental data, the curves are results from model calculations. 


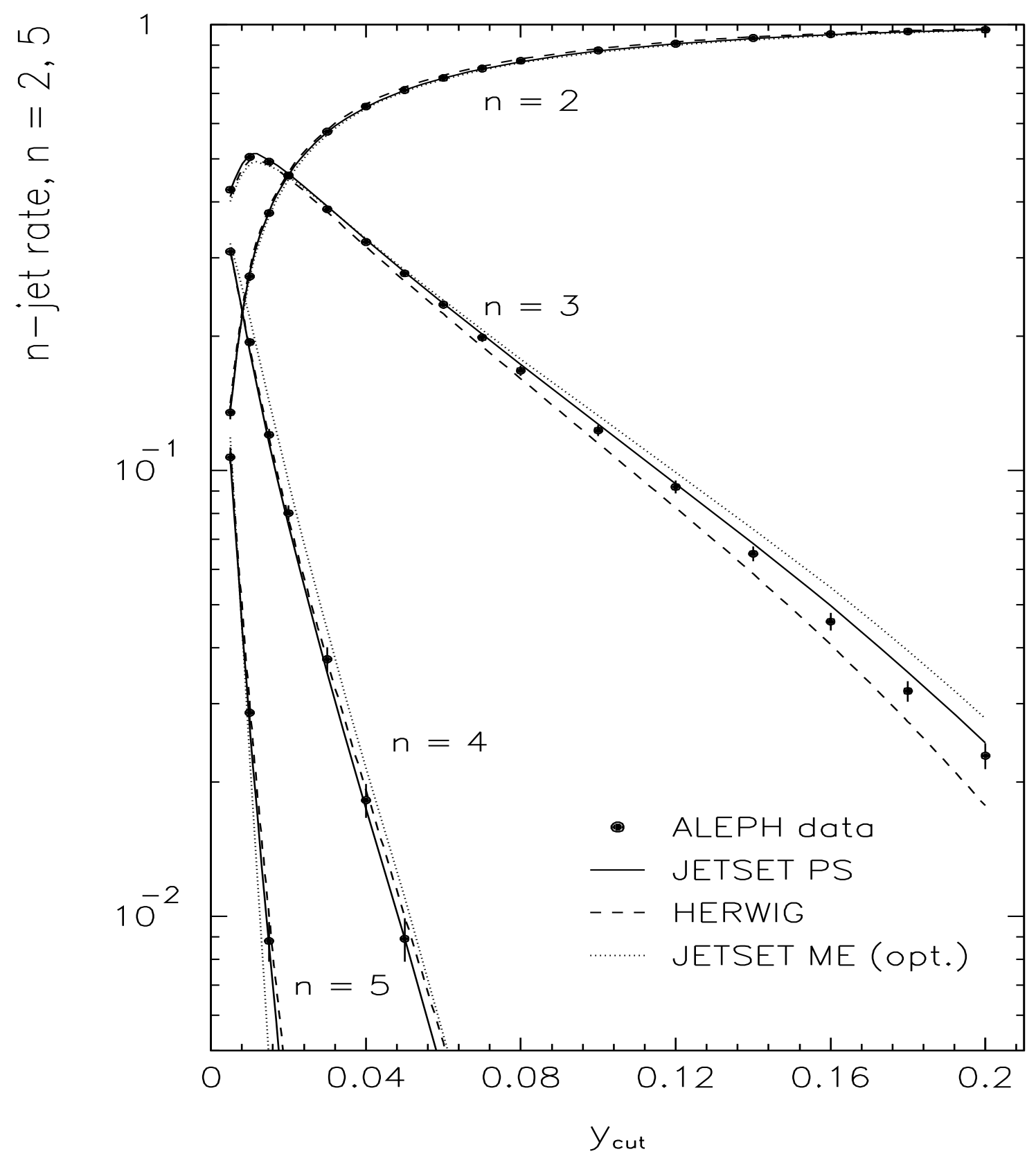

Figure 8: Jet rates determined by the ALEPH-collaboration [29] as function of the jet resolution parameter $y_{c u t}$. The experimental results are compared to model calculations. Note that neighbouring points are highly correlated. 


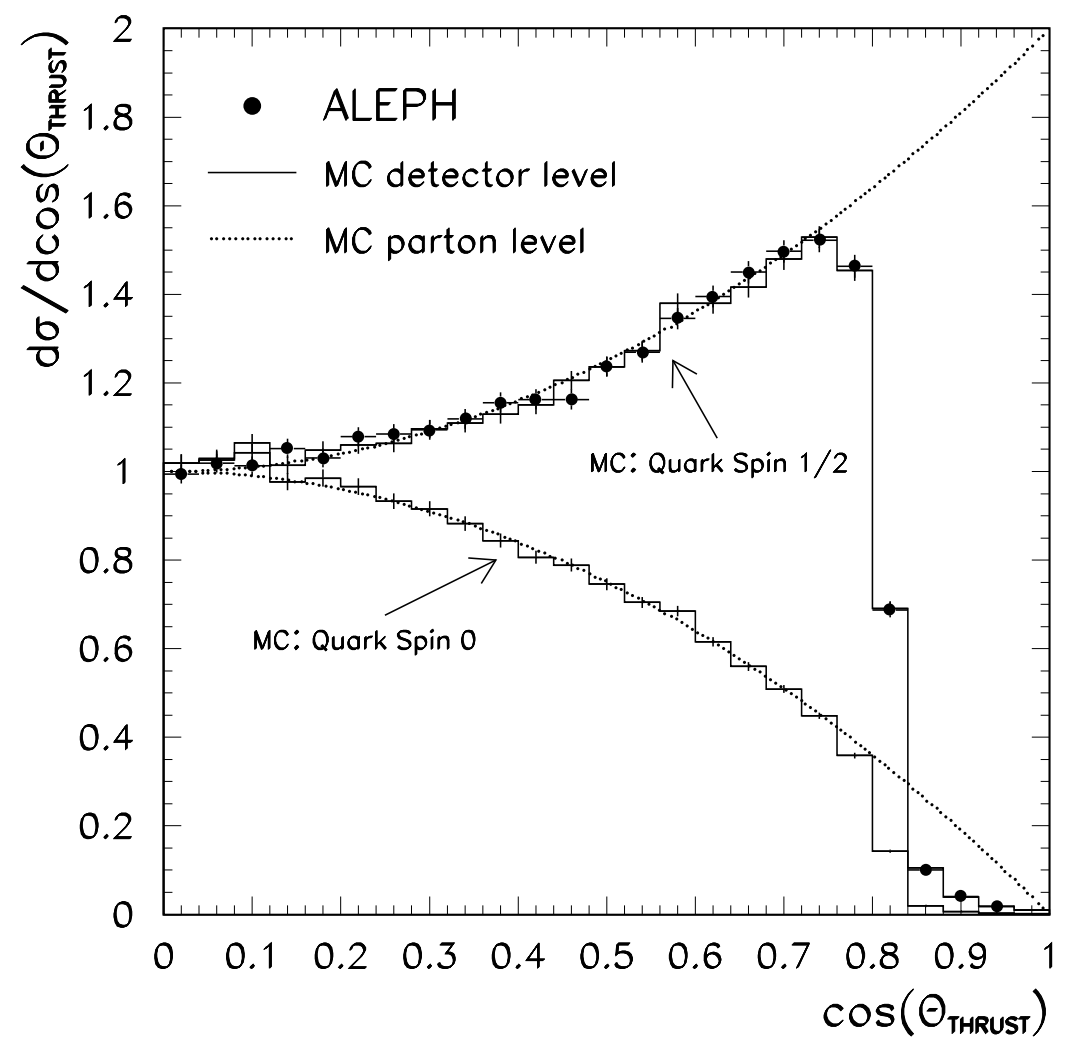

Figure 9: Angular distribution of the Thrust axis. The drop in the data above $\cos \Theta \approx 0.8$ is due to the event selection procedure.
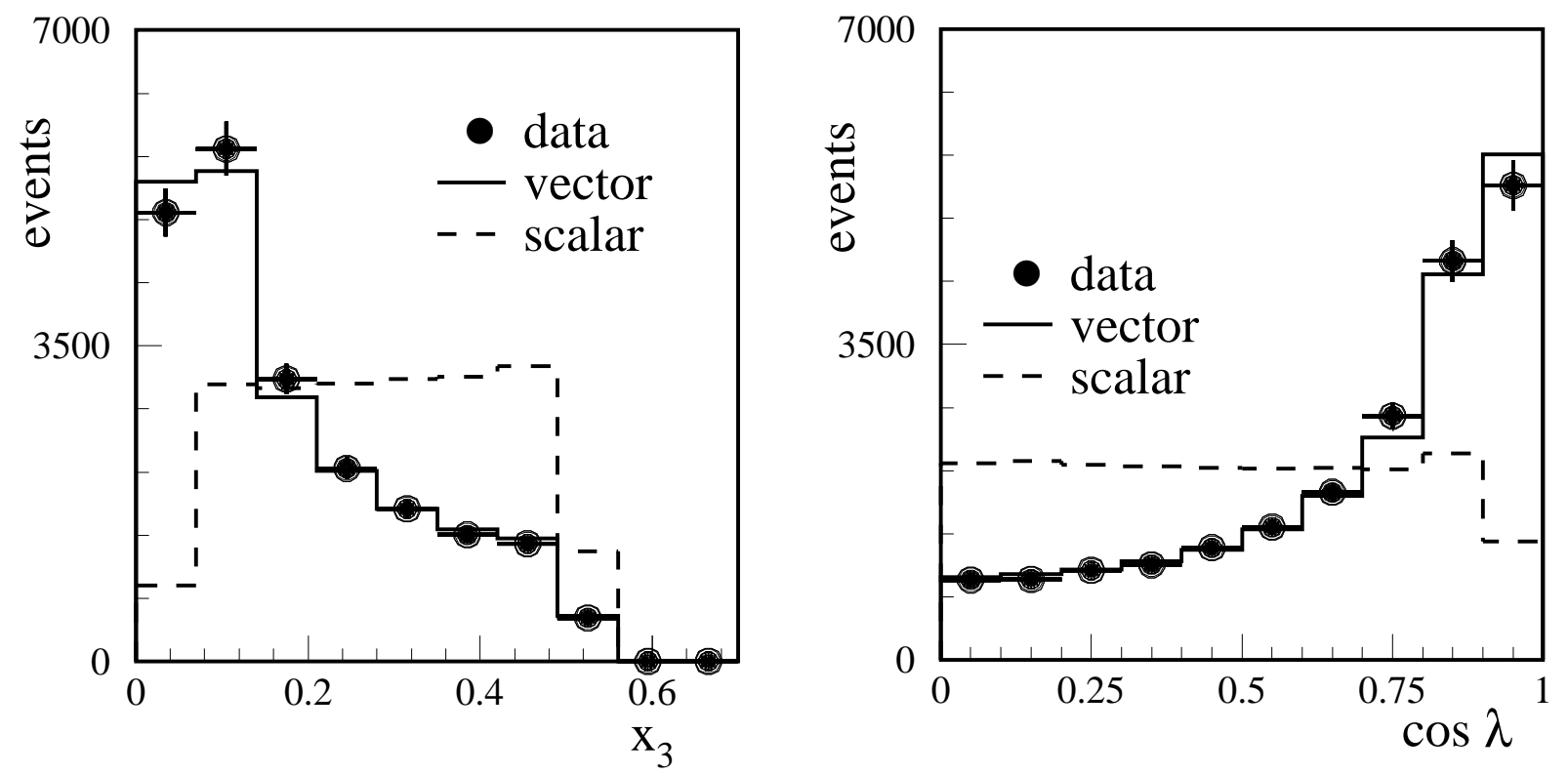

Figure 10: Distributions sensitive to the gluon spin as measured by the L3-collaboration [38]. The experimental data are compared to predictions from a vector- and a scalar gluon model. 


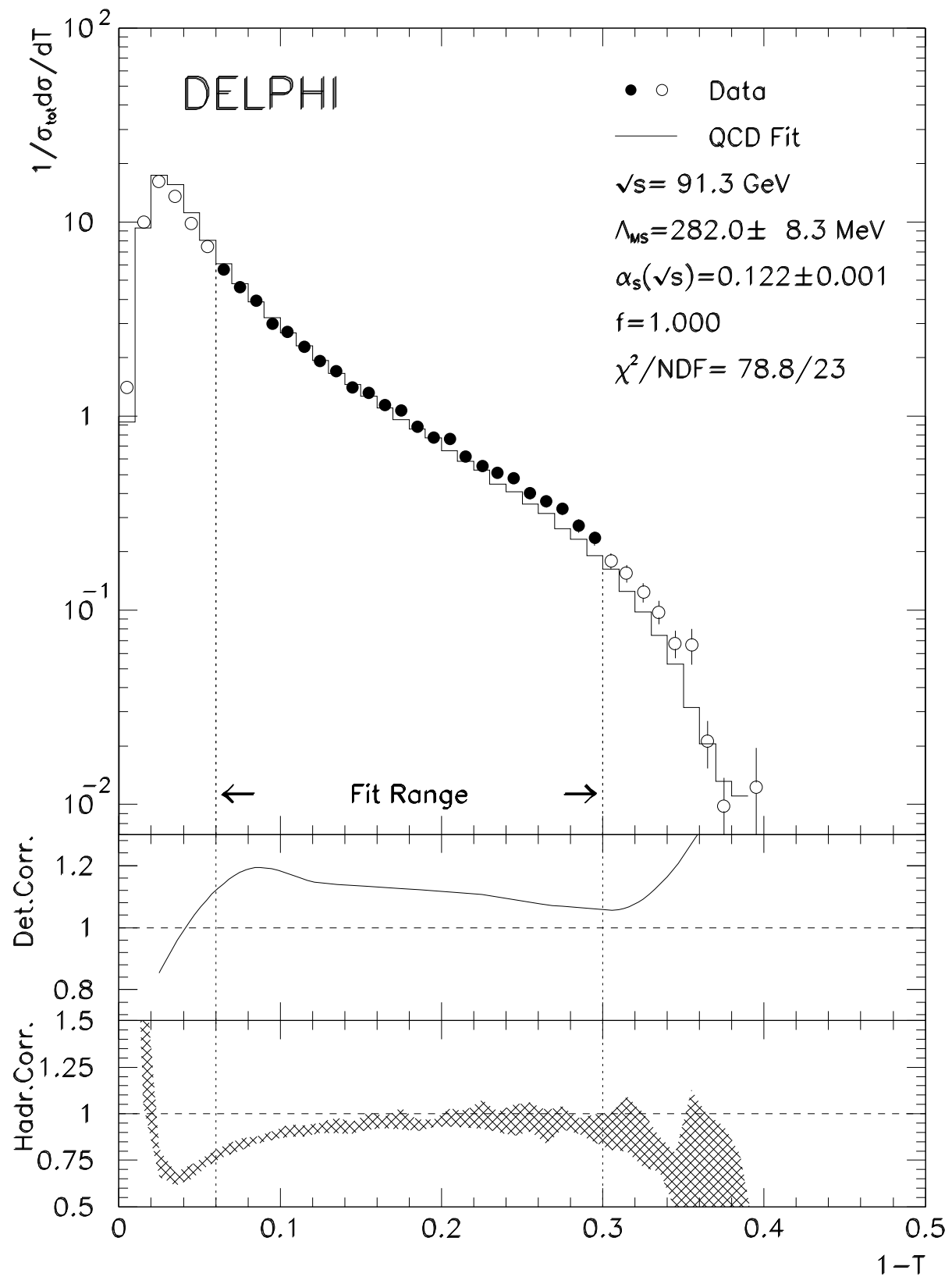

Figure 11: Measurement of the strong coupling constant from the Thrust distribution. The data points used in the fit are indicated by the full dots. Detector and hadronization corrections are indicated below. The theoretical prediction is the second order matrix element plus resummation of leading and next-to-leading logarithms. 


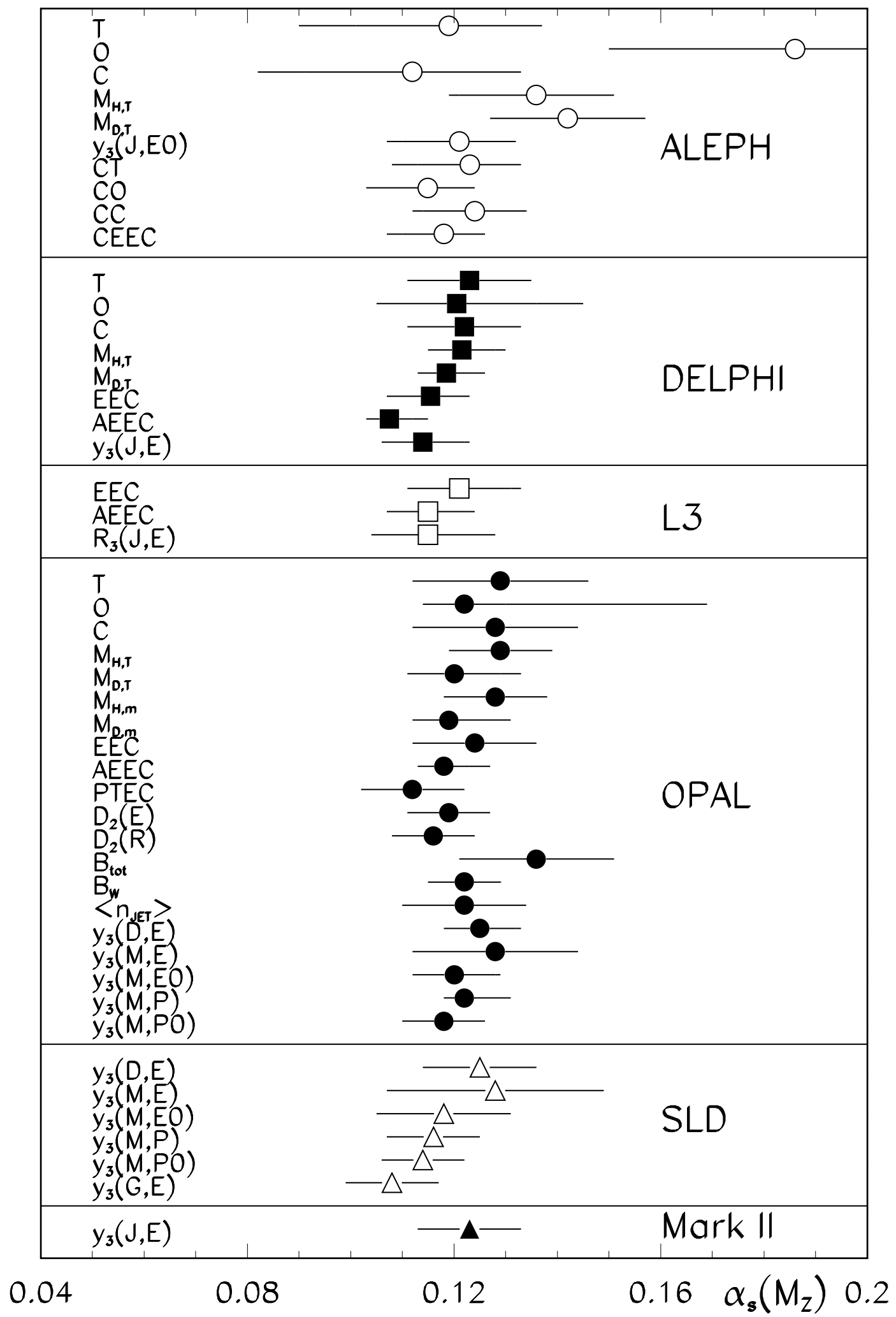

Figure 12: Measurements of the strong coupling constant from event shape variables based on second order QCD predictions. 


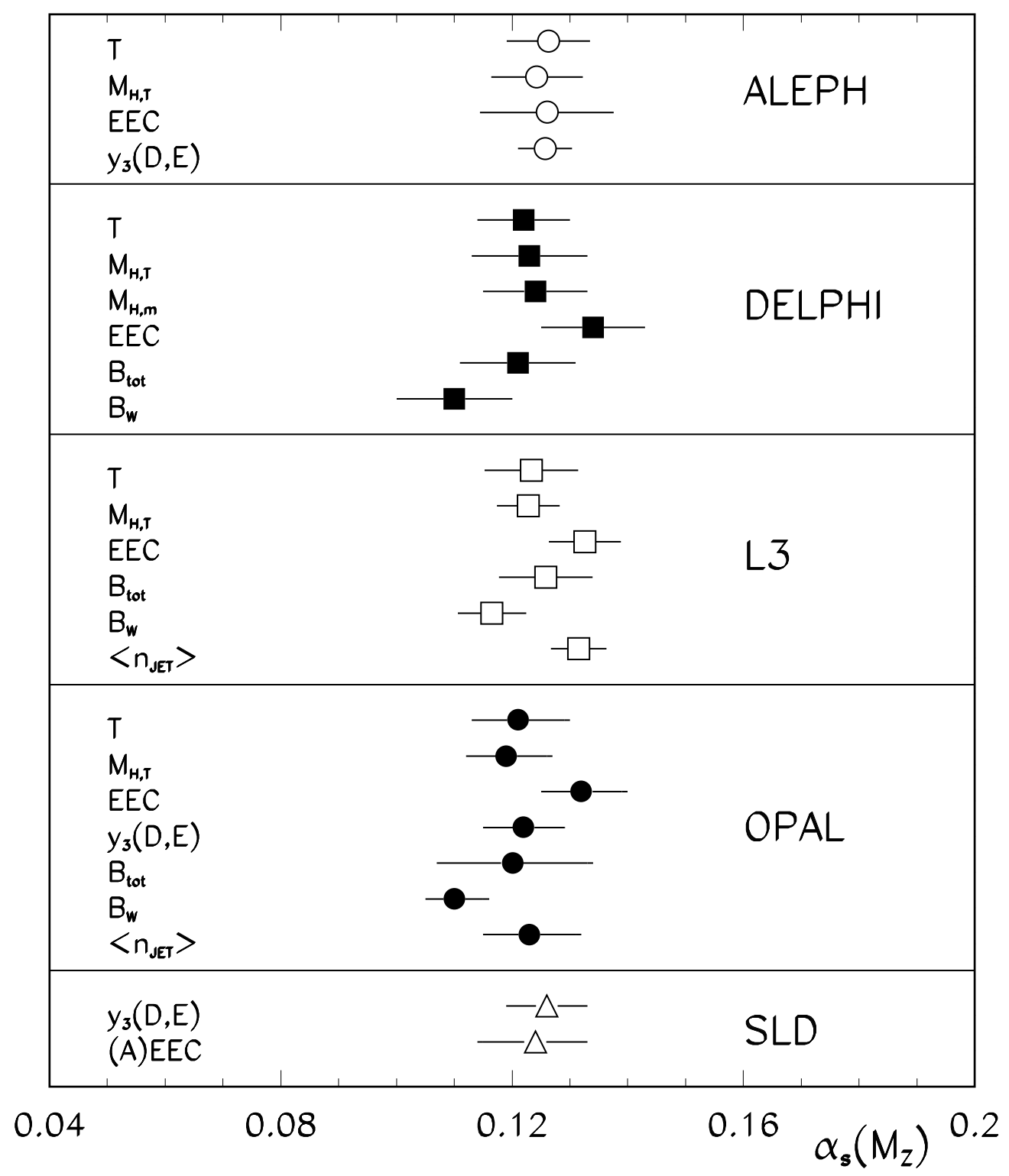

Figure 13: Measurements of the strong coupling constant from event shape variables based on the improved theoretical prediction combining second order matrix elements with resummed leading-log and next-to-leading log predictions. 


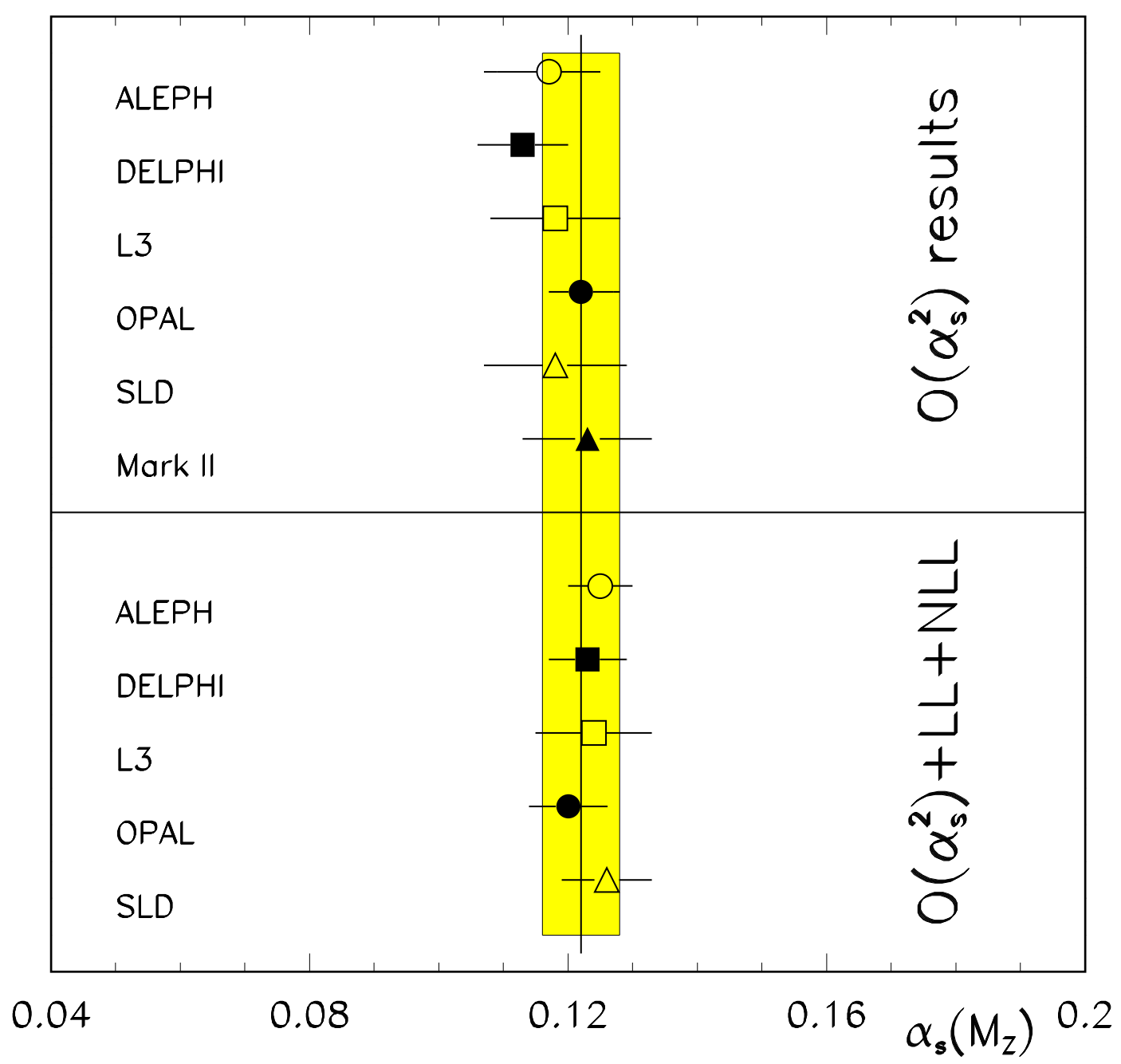

Figure 14: Measurements of the strong coupling constant obtained by combining information from various event shape variables. Results are given both for the purely second order QCD predictions and improved resummed predictions. The shaded region indicates the error band estimated [83] for a global average based on these results. 


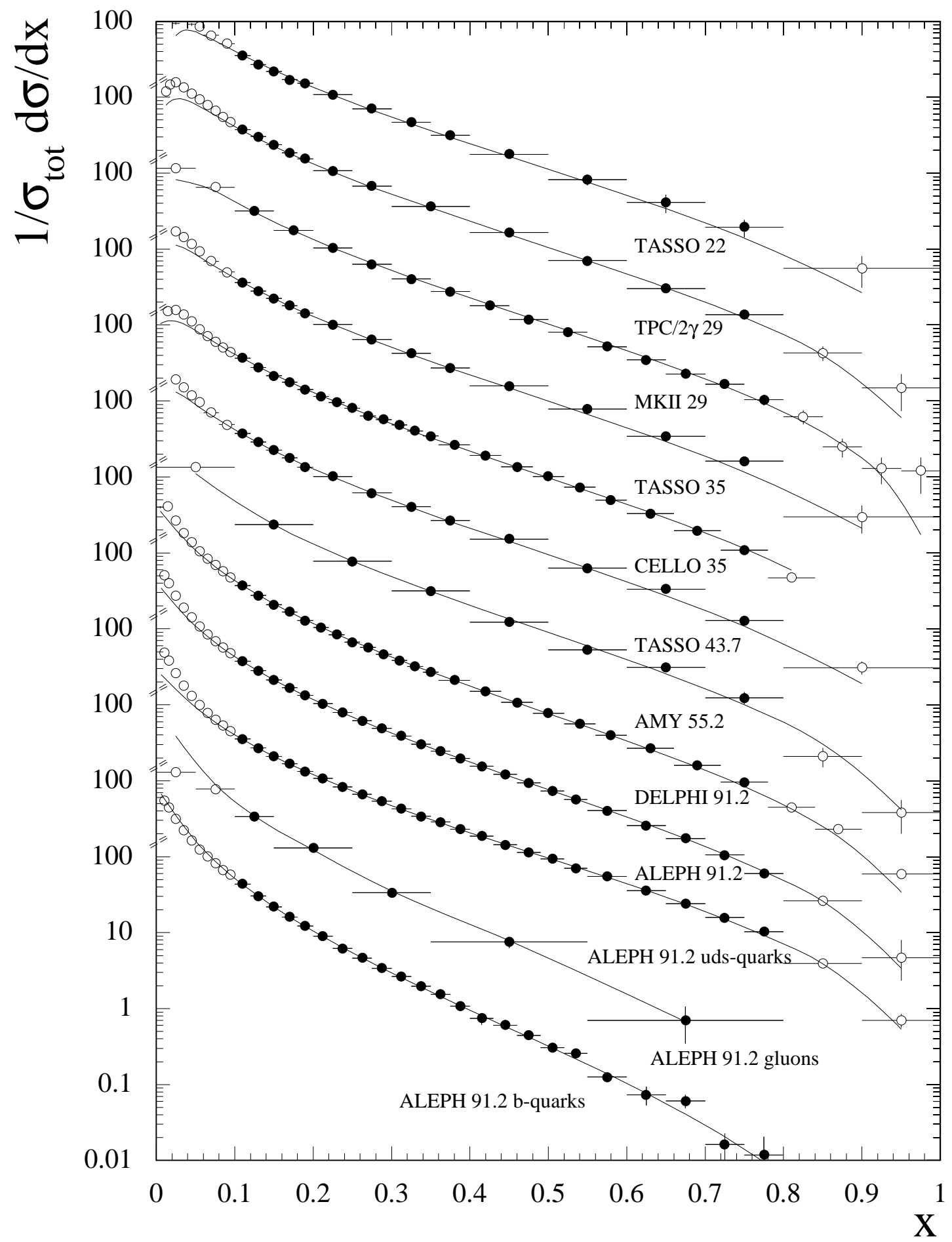

Figure 15: Analysis of scaling violations in fragmentation functions. Measured $x-$ distributions for the natural flavour mix at energies between $22 \mathrm{GeV}$ and $91.2 \mathrm{GeV}$, and data for pure $u d s-, b$-quark and gluon samples at $91.2 \mathrm{GeV}$ are compared to the result of a global QCD fit. The full dots contribute to the fit, the continous lines represent the result. 


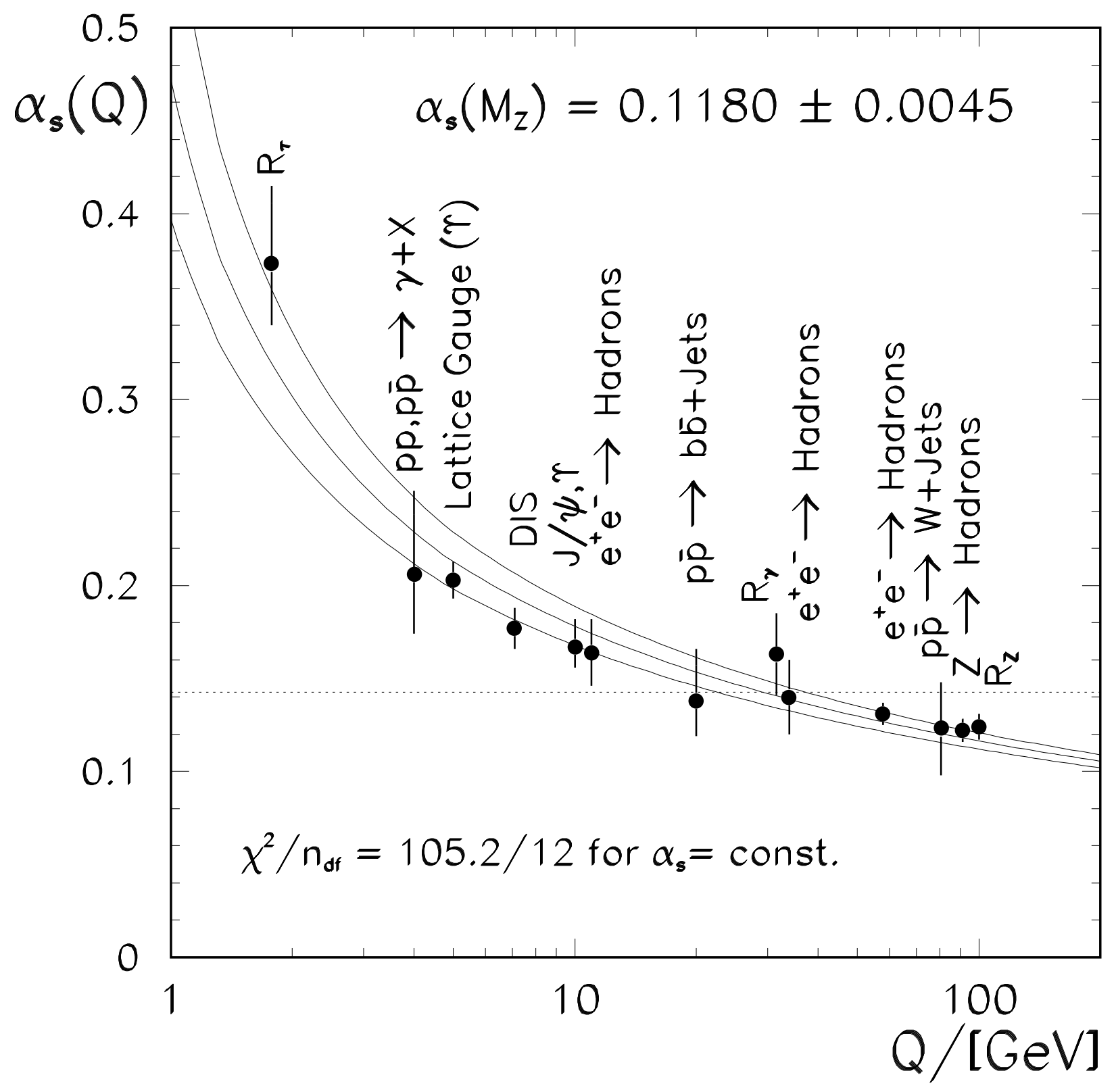

Figure 16: Measurements of the strong coupling constant at various energy scales. The curves show the QCD prediction for the running with $\alpha_{s}\left(M_{Z}\right)=0.1180 \pm 0.0045$. The dotted line is a simple weighted average of the individual results, which is clearly incompatible with the data. 


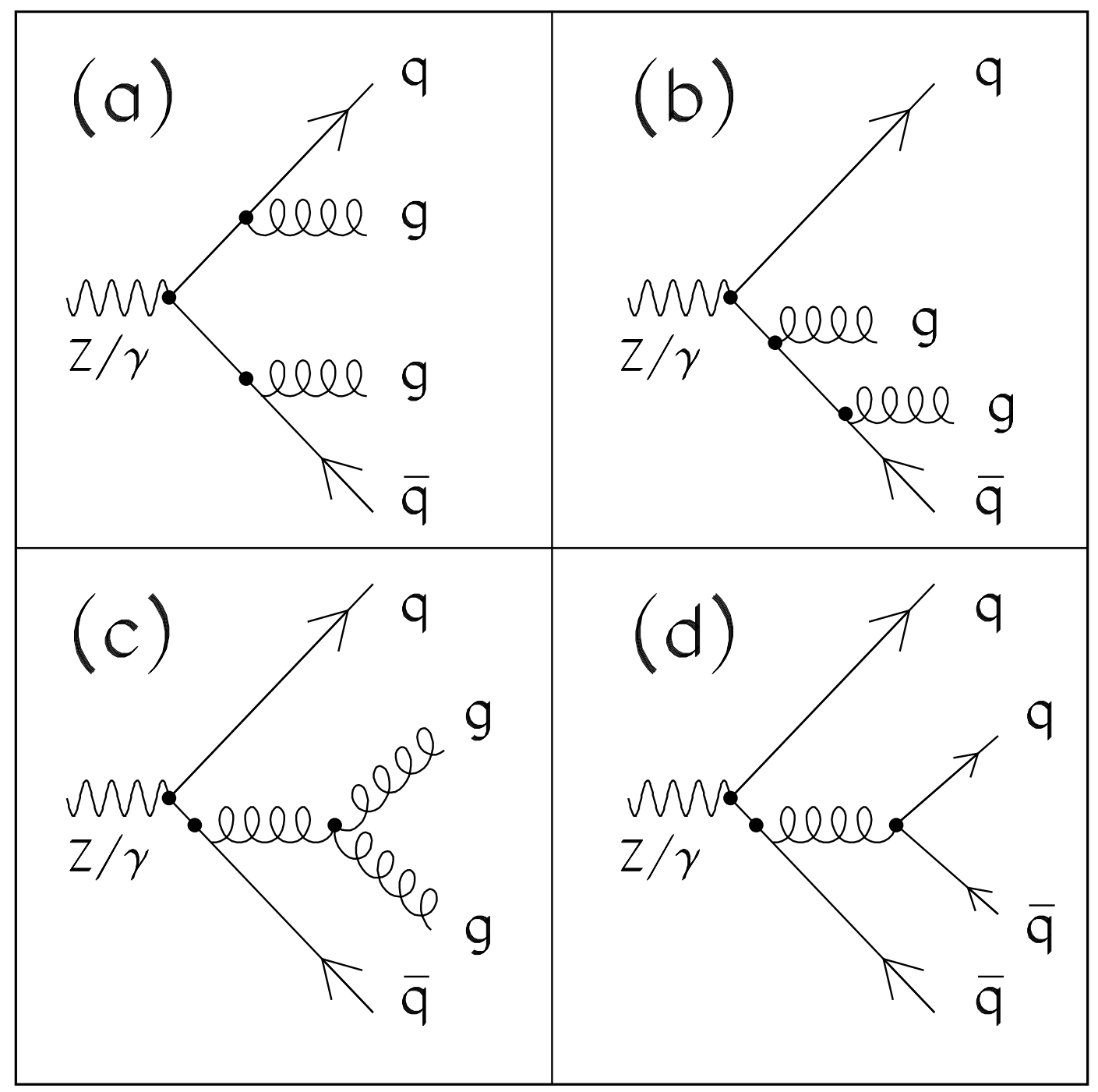

Figure 17: Generic $\mathrm{O}\left(\alpha_{s}^{2}\right)$-contributions to multihadron production from a virtual photon or a $Z$. 


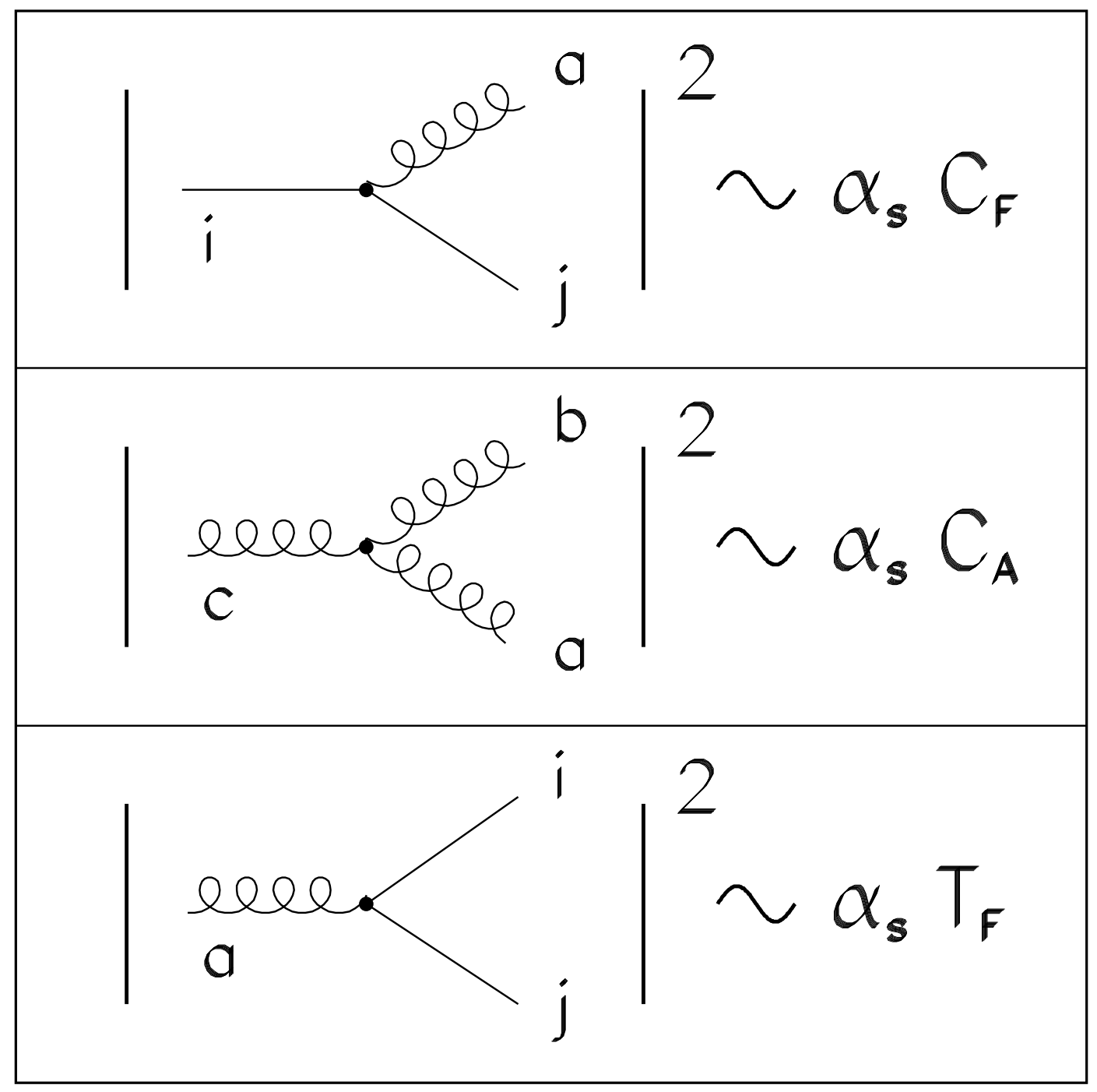

Figure 18: Definition of the colour factors as the basic probabilities for a quark to radiate a gluon $\left(C_{F}\right)$, a gluon to radiate a gluon $\left(C_{A}\right)$ or a gluon to split into secondary quarks $\left(T_{F}\right)$. Note that $C_{F}$ and $T_{F}$ are not independent as they are defined by topologically equivalent diagrams. 


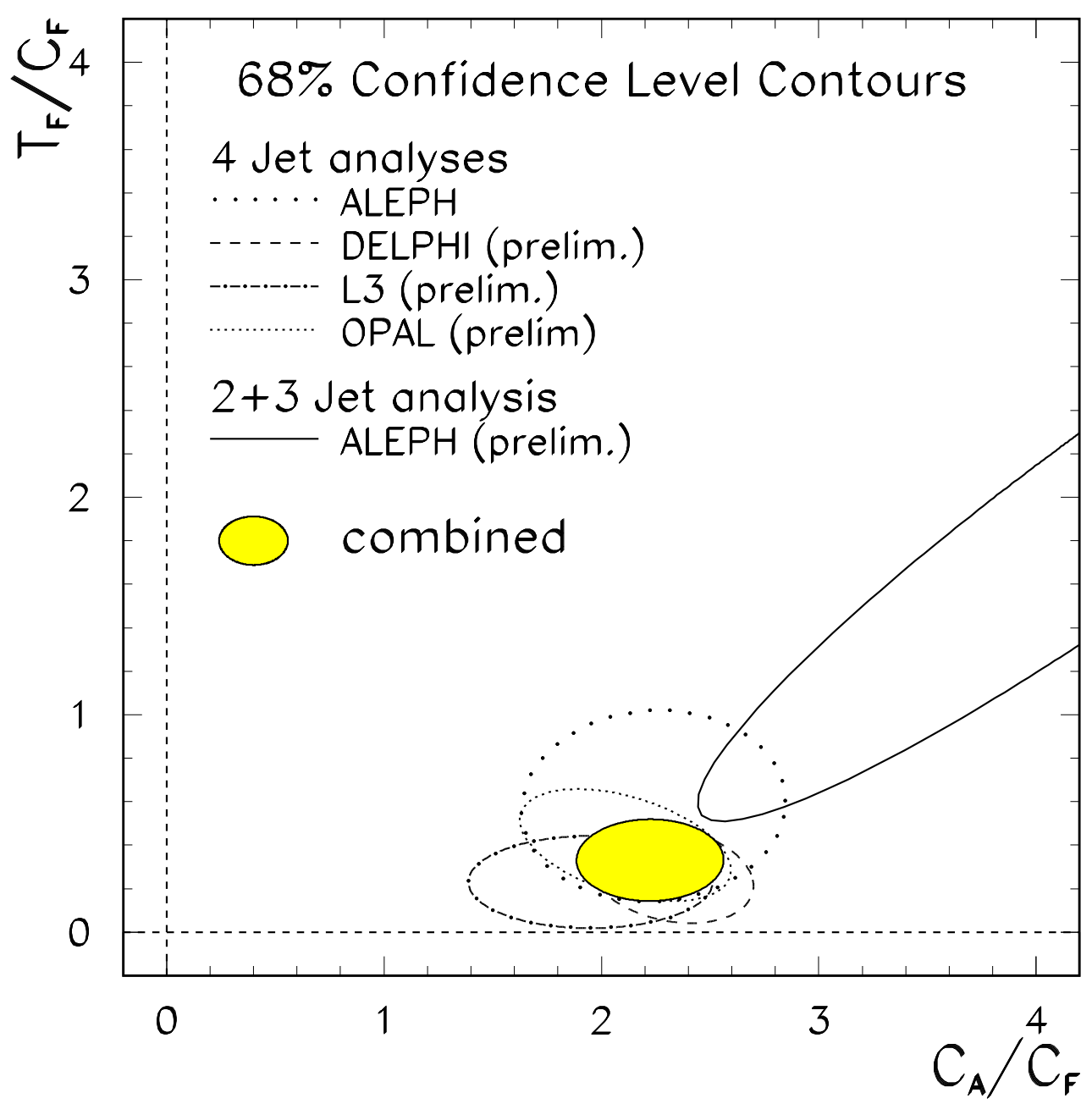

Figure 19: Colour factor measurements from jet studies done at LEP. The $68 \%$ confidence level contours from the analysis of 2-, 3- and 4-jet events are plotted in the $C_{A} / C_{F}$ versus $T_{F} / C_{F}$ plane. The shaded ellipse is the combined result. 


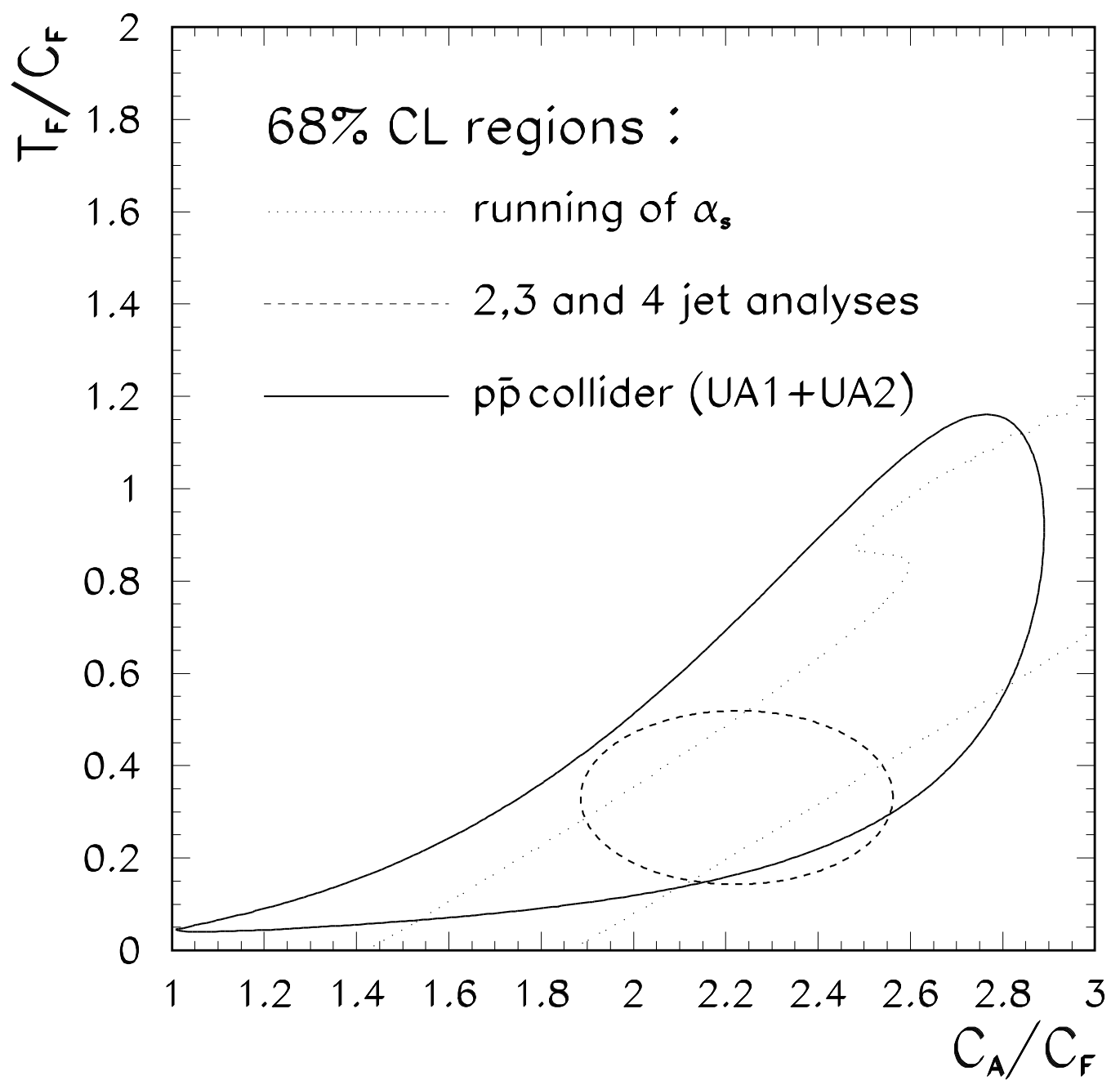

Figure 20: Comparison of colour factor measurements from different sources. In addition to the combined result from multi-jet events at LEP the results from an analysis of the running of the strong coupling, based on $R_{Z}$ and $R_{\tau}$, and from jet production in proton-antiproton collisions is shown. 


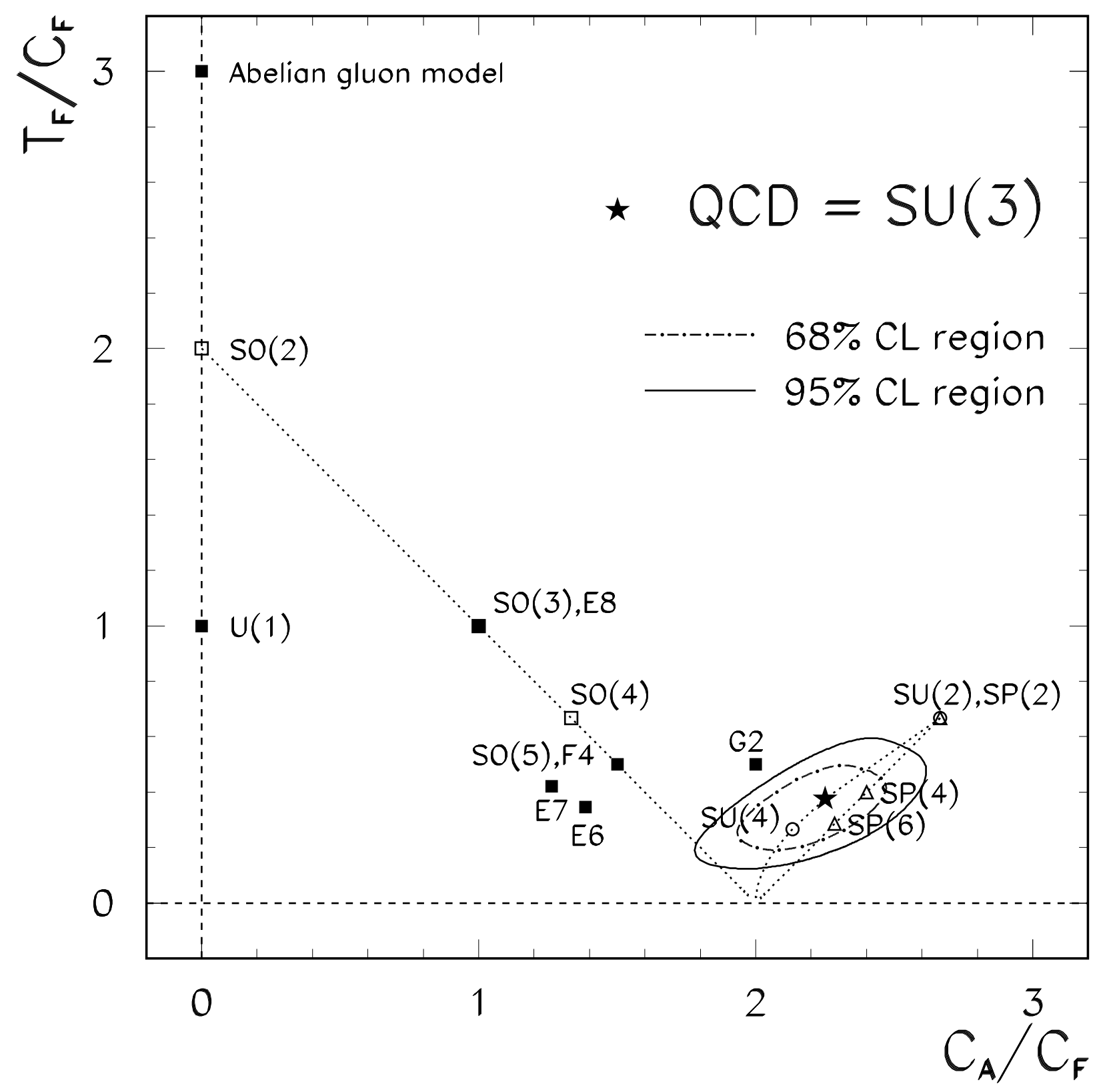

Figure 21: Combined result from all colour factor measurements. The $68 \%$ and $95 \%$ confidence level contours are plotted together with the expectation for all simple Lie-groups with the fermions in the fundamental and the gluons in the adjoint representation. 

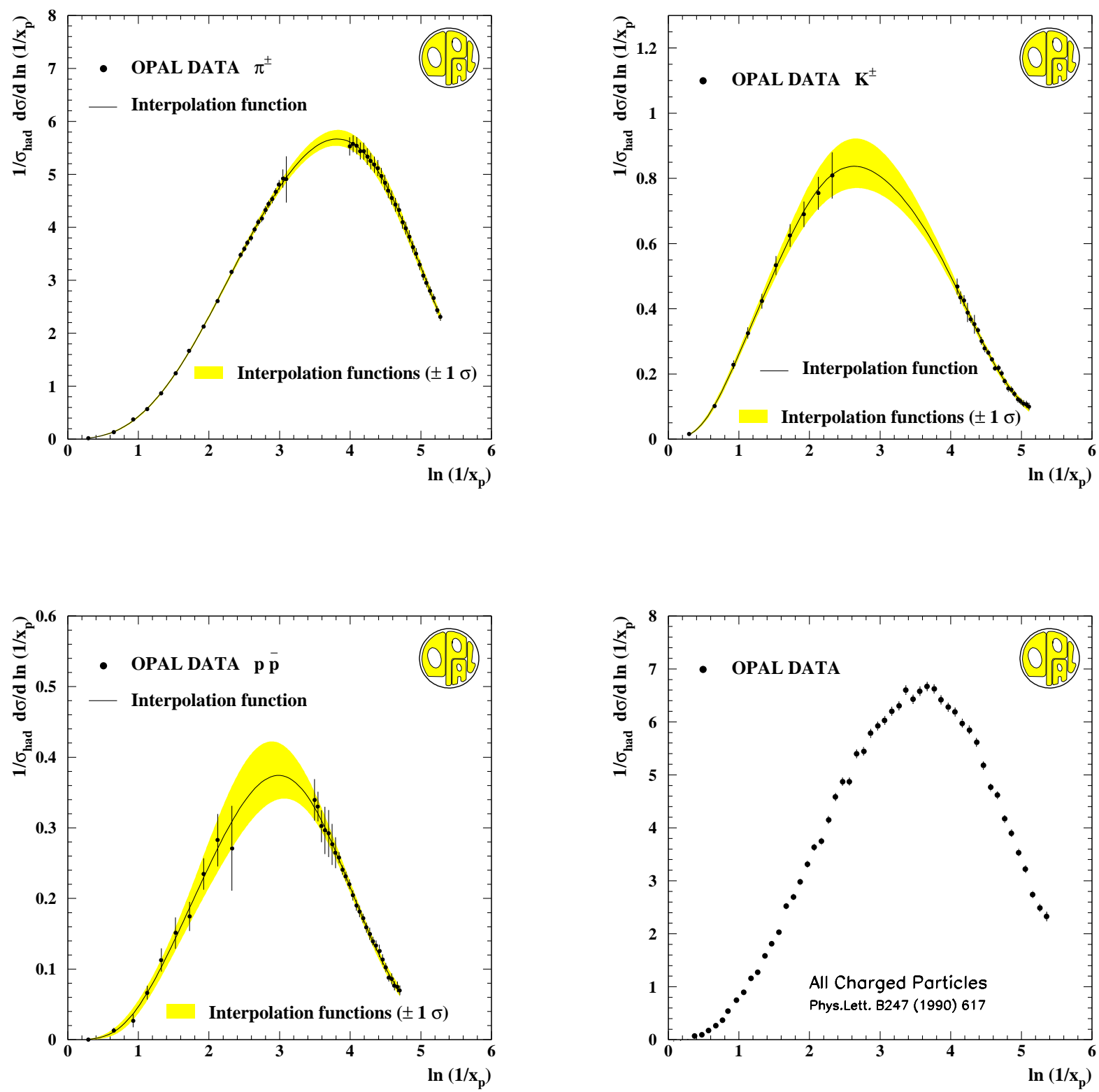

Figure 22: $\ln \left(1 / x_{p}\right)$ distributions for charged hadrons measured by the OPALcollaboration. The lower right graph is for the inclusive spectrum of all charged hadrons, taken from [168], the other ones from [169] are for identified particles. The continuous lines are the results from a QCD fit to the data, based on the MLLA and LPHD theoretical prediction. 

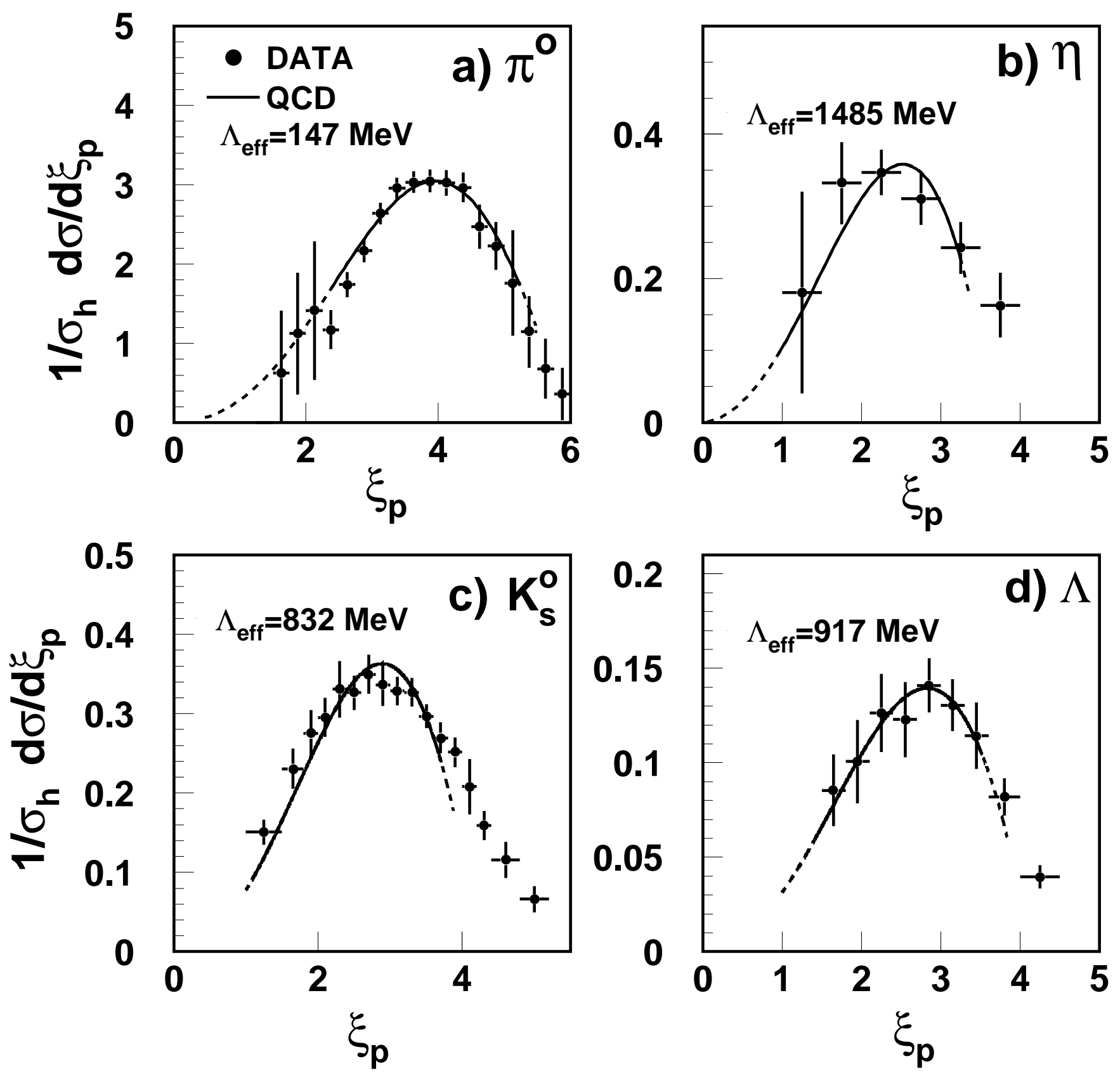

Figure 23: $\ln \left(1 / x_{p}\right)$ distributions for identified neutral hadrons determined by the L3-collaboration [170]. The curves are QCD fits using the MLLA+LPHD theoretical prediction. The resulting values for $\Lambda_{\text {eff }}$ are indicated in the individual figures. 


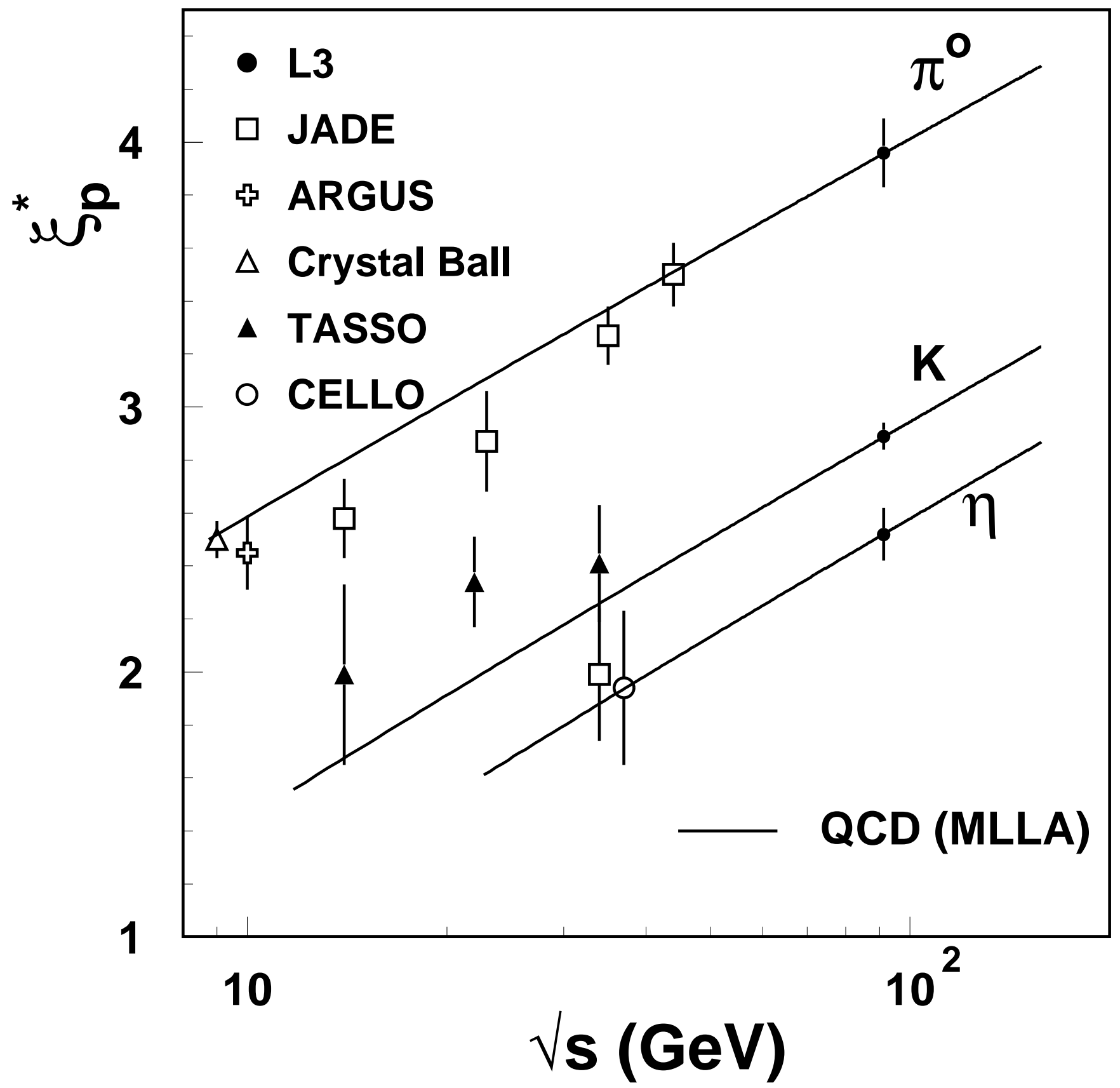

Figure 24: Dependence of the peak position on the centre-of-mass energy for identified neutral hadrons. The lines show the MLLA prediction using the values $\Lambda_{\text {eff }}$ determined from the $x i$-spectra fig.23 [170] at $E_{c m}=91.2 \mathrm{GeV}$. 


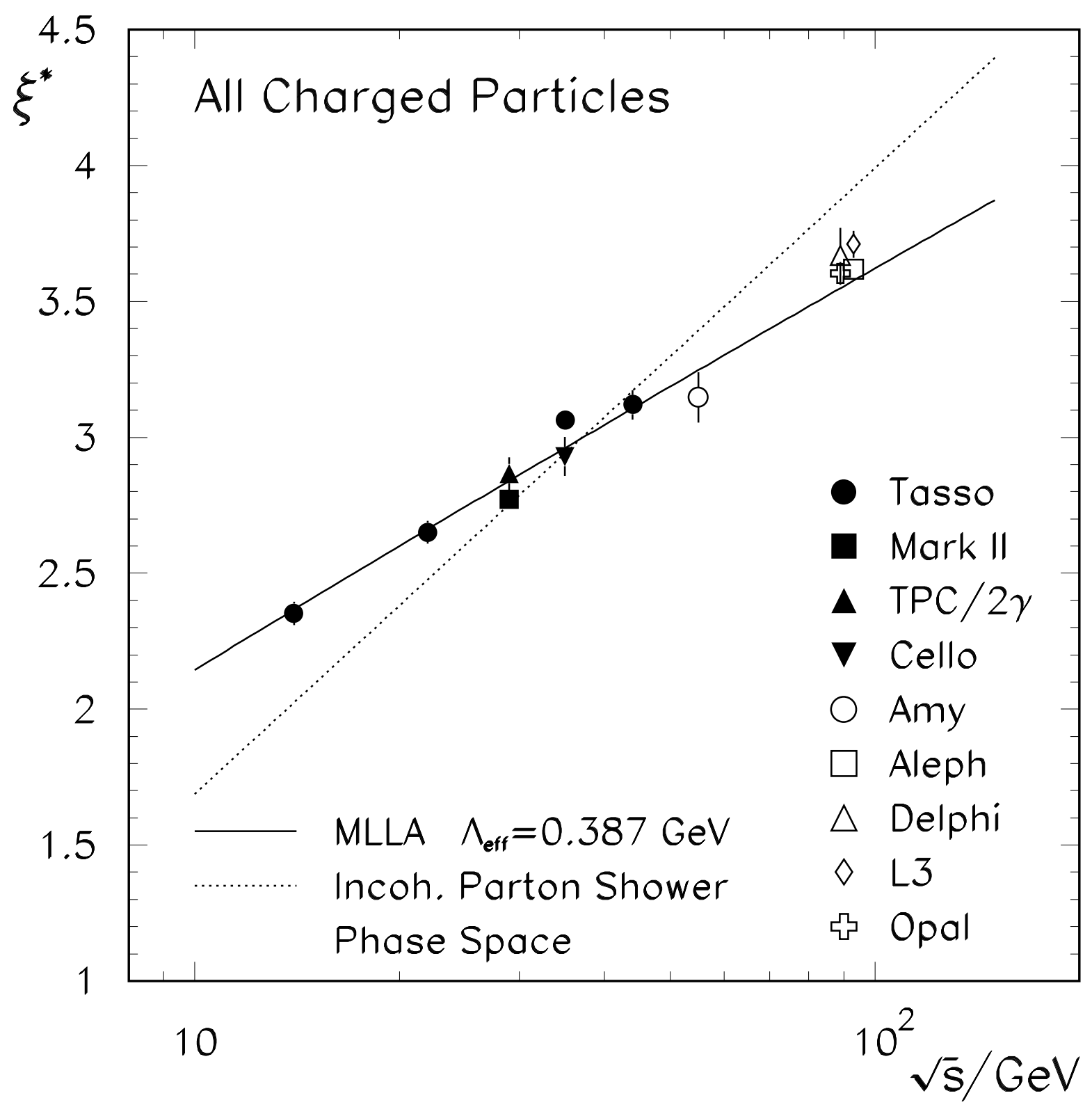

Figure 25: Peak position $\xi^{\star}$ of the inclusive $\xi$-spectra of all charged particles as function of the centre-of-mass energy. The full line is a fit to the MLLA prediction, the dotted line represents the expectation for an incoherent parton shower or a simple phase space model. 


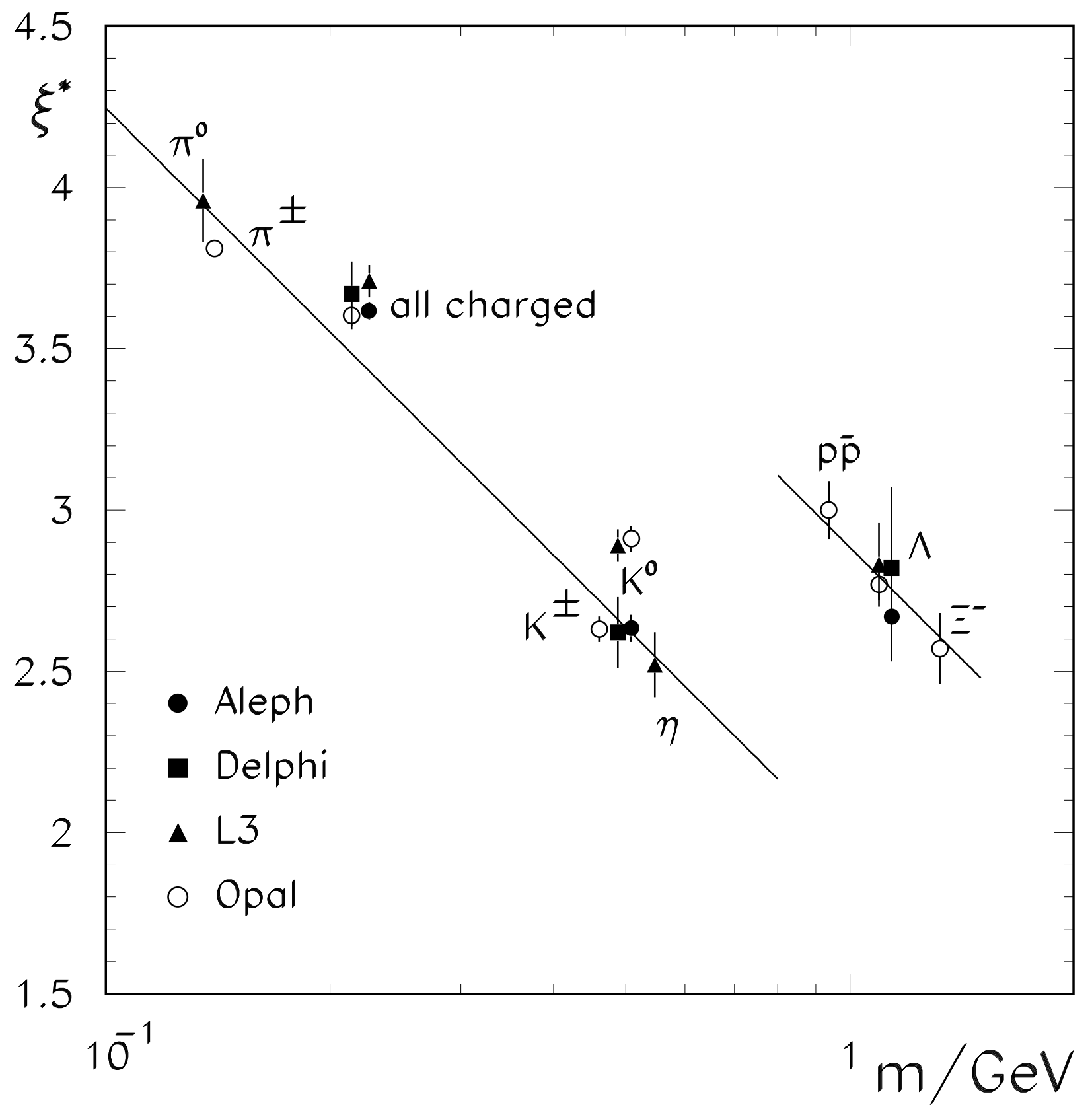

Figure 26: Relation between the peak position of the $\xi$-distribution at $E_{c m}=M_{Z}$ and the mass of the hadron. The lines are fits to the data using the functional form $\xi^{\star}=\ln m_{0} / m$ with different parameters $m_{0}$ for mesons and baryons. For better visibility measurements for the same particle species from different experiments are plotted slightly displaced from the true mass value. Note that despite qualitative agreement there are some discrepancies in the $K^{0}$-results obtained by the different LEP experiments. 


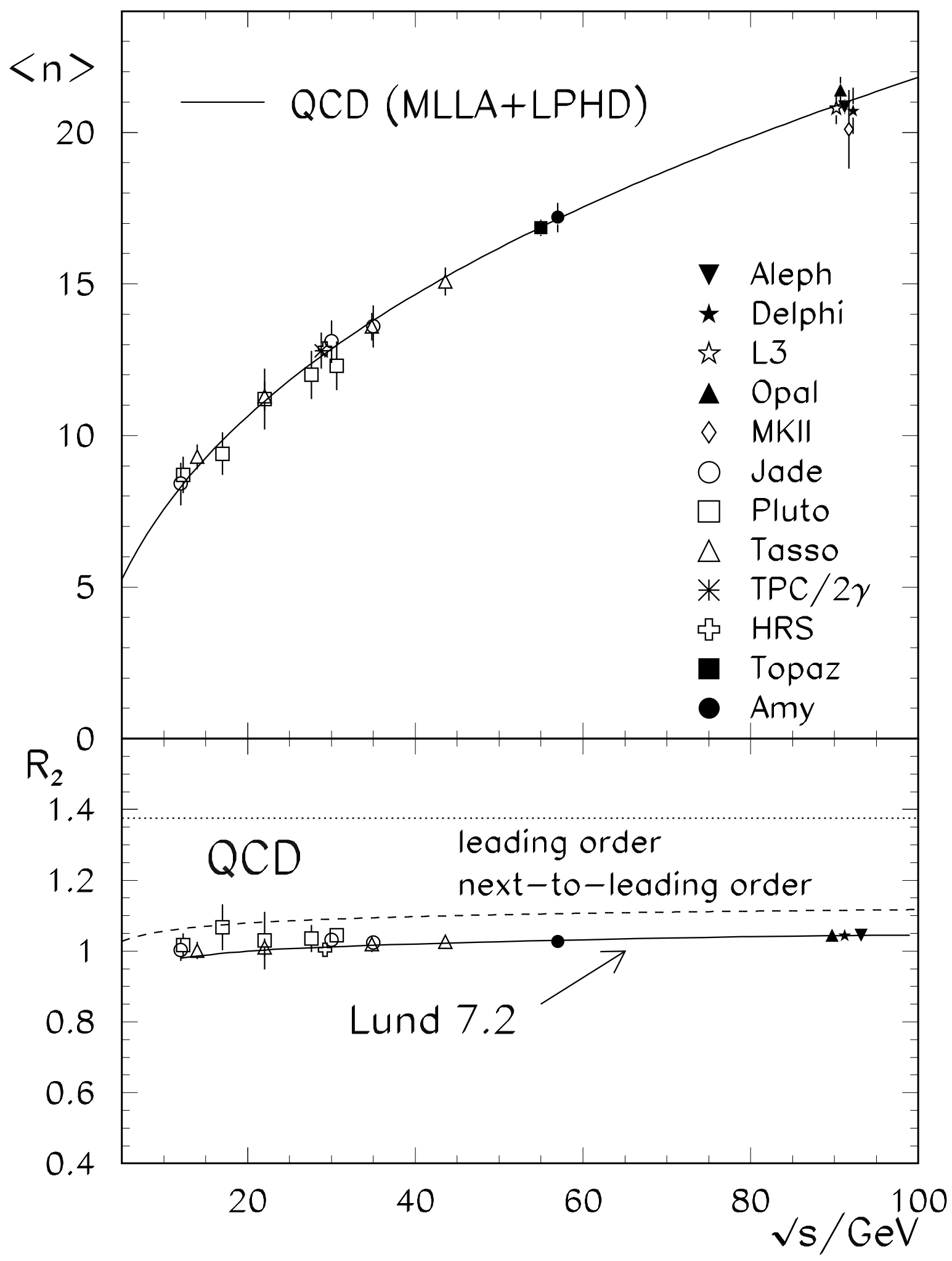

Figure 27: Energy dependence of the mean charged particle multiplicity $<n>$ and the second binomial moment $R_{2}$. The experimental data are compared to QCD predictions obtained in the framework of MLLA+LPHD. 


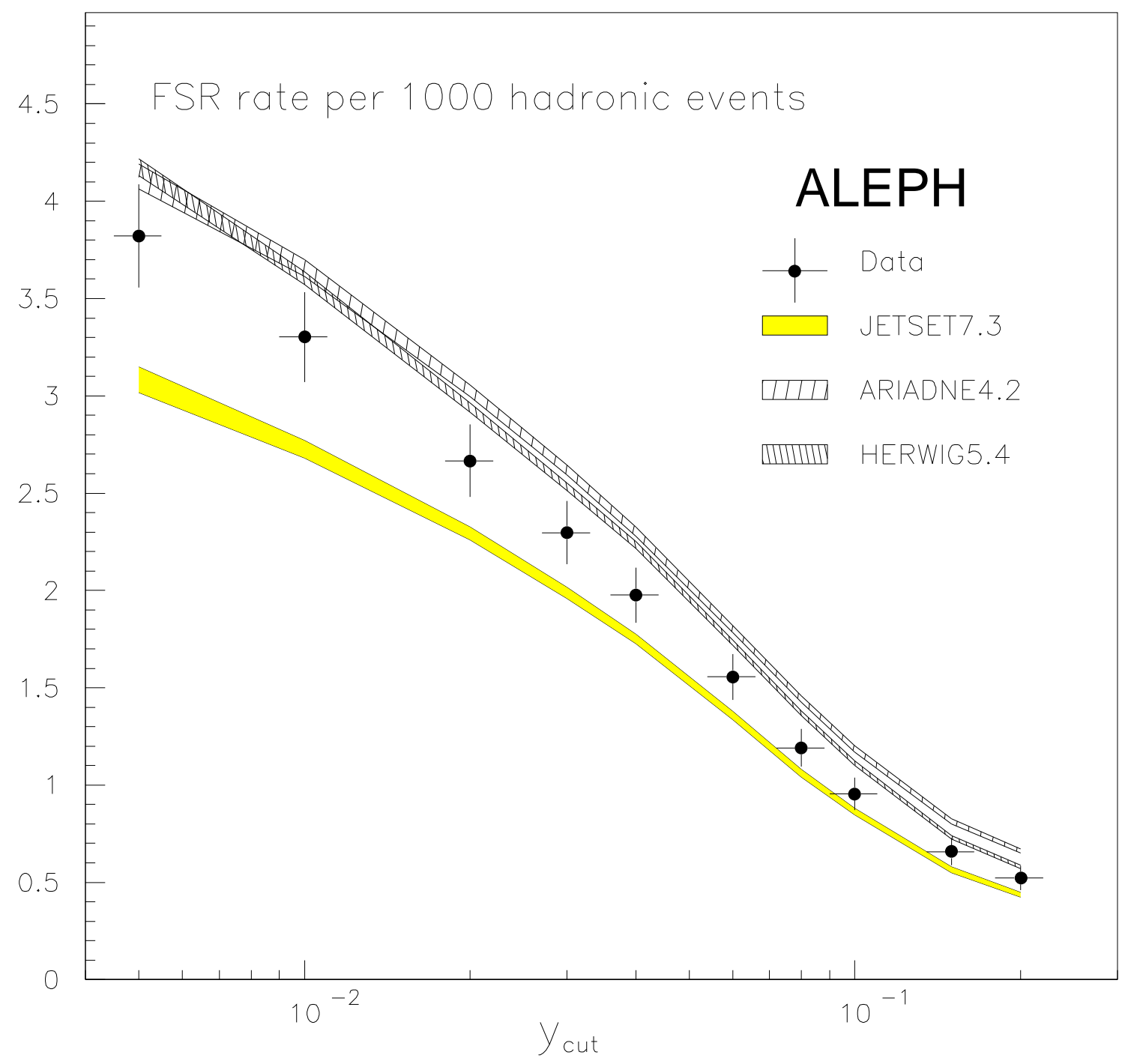

Figure 28: Production rates for isolated photons with an energy above $5 \mathrm{GeV}$ measured by the ALEPH collaboration [205] as function of the isolation parameter $y_{\text {cut }}$. The photon was considered isolated at a resolution $y_{\text {cut }}$, if its distance to the closest jet according to the JADE-metric was greater than $y_{\text {cut }}$ with the jets defined by the JADE algorithm applied to all final state particles except the photon. The measurements are compared to expectation from various parton shower models that were tuned to describe the global features of hadronic $Z$-decays. 

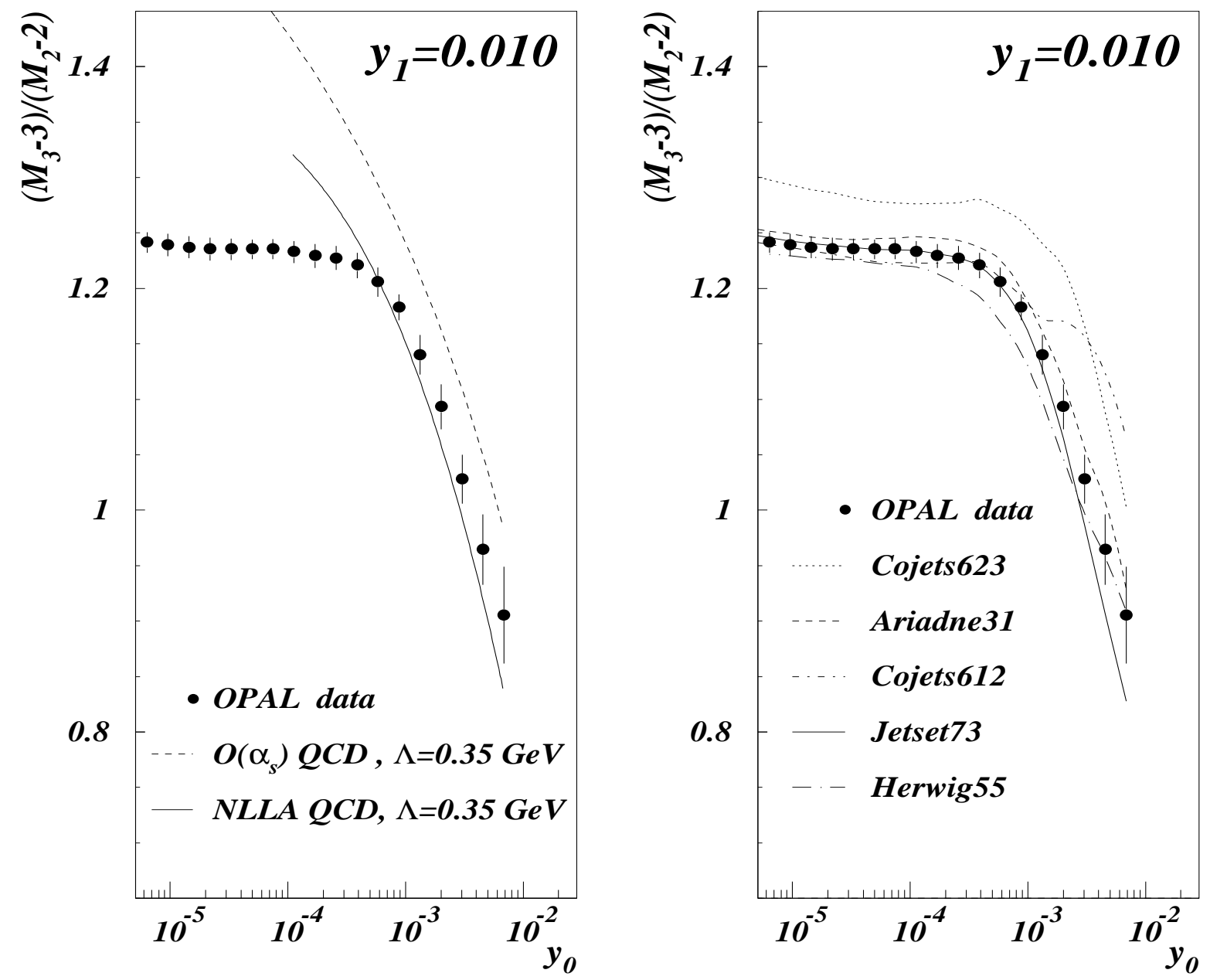

Figure 29: Ratio of subjet multiplicities in events with two or three jets at an initial resolution parameter $y_{1}=0.01$ [208] as function of the secondary resolution $y_{0}$. The data are compared to theoretical predictions from perturbative QCD (left) and different Monte-Carlo models (right). 


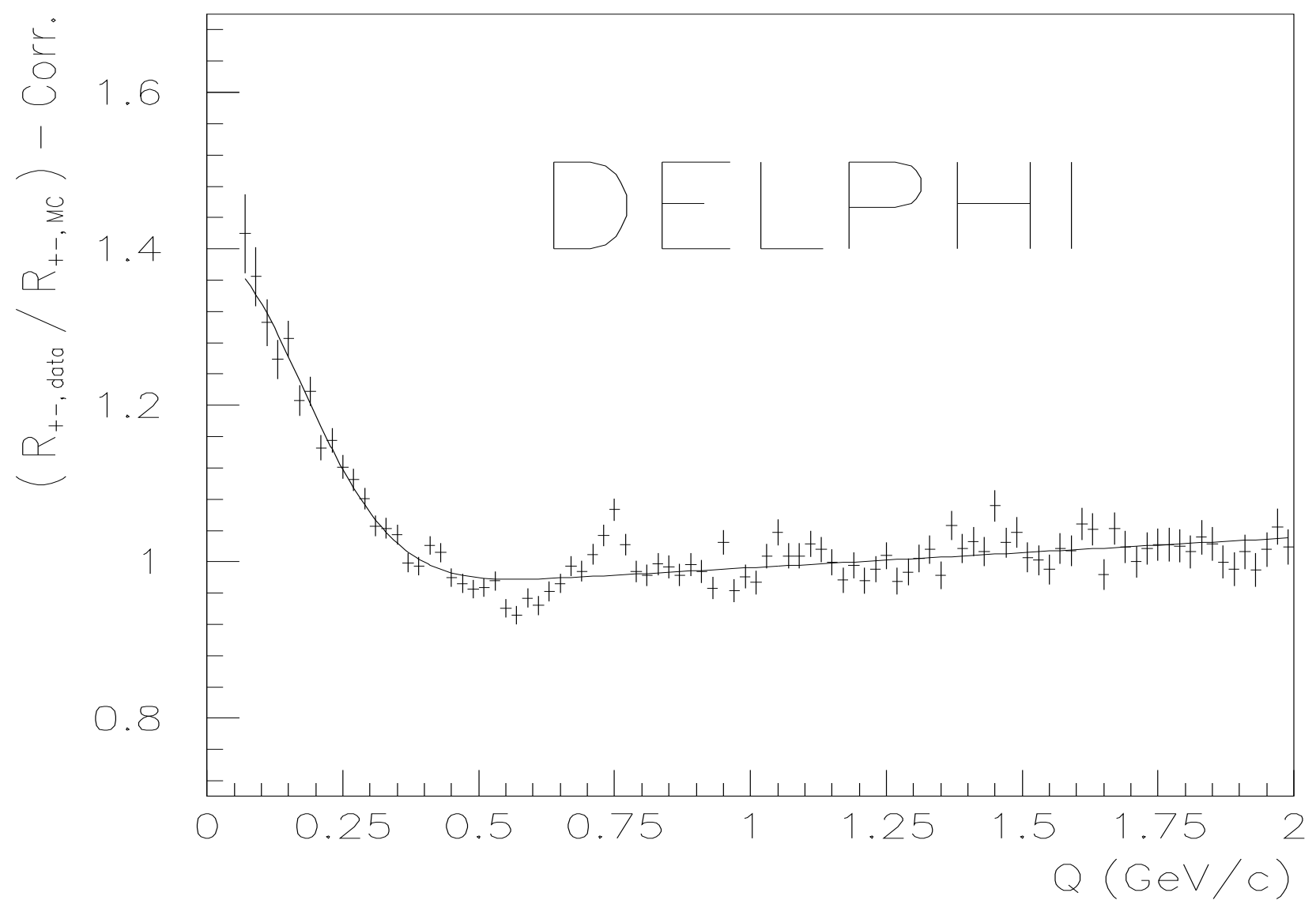

Figure 30: Bose-Einstein correlations measured for charged pions [209]. The continous line shows the parametrization of the correlation function. 


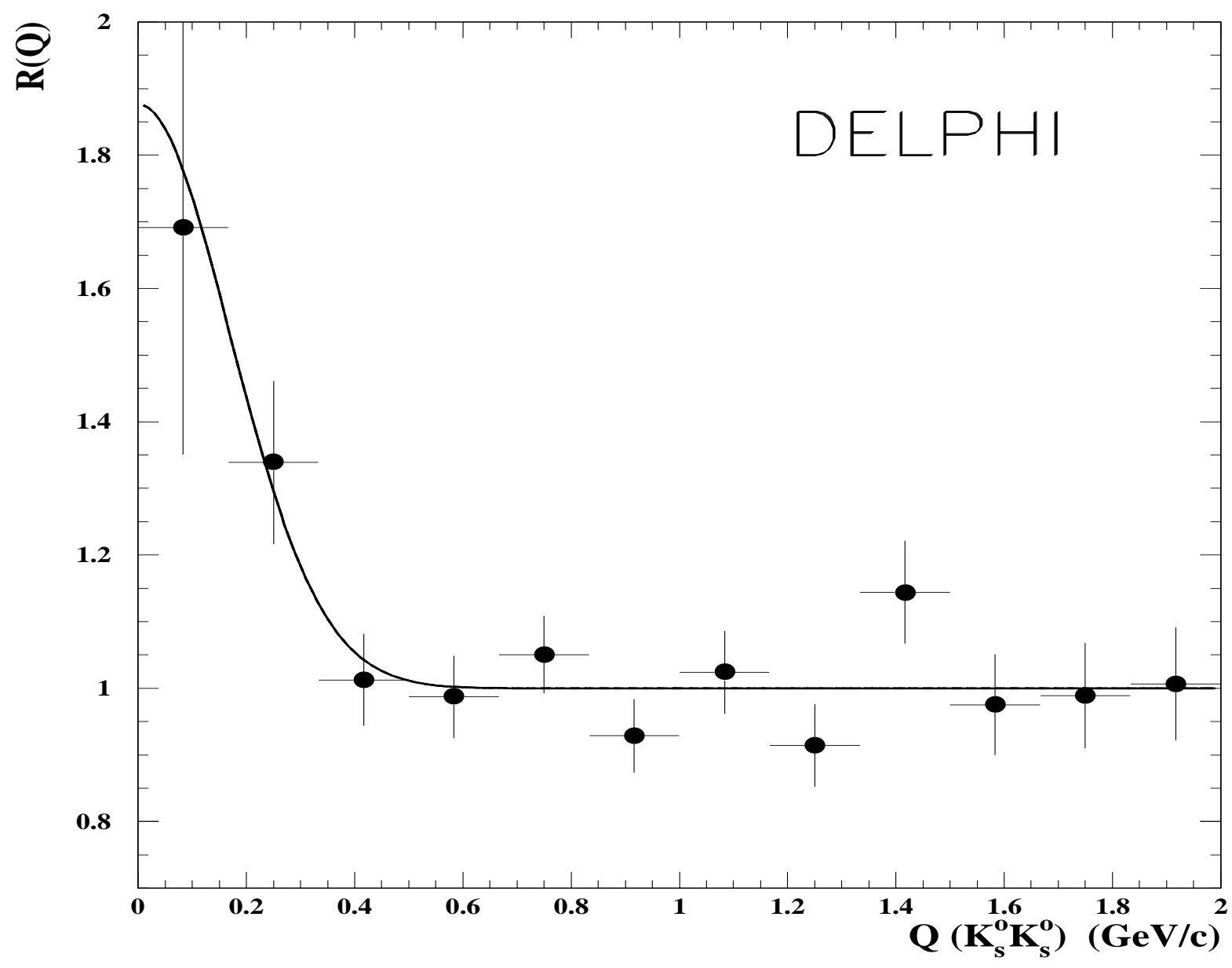

Figure 31: Bose-Einstein correlations observed for neutral kaons [210]. The continous line shows the fitted correlation function in comparison to the experimental data. 


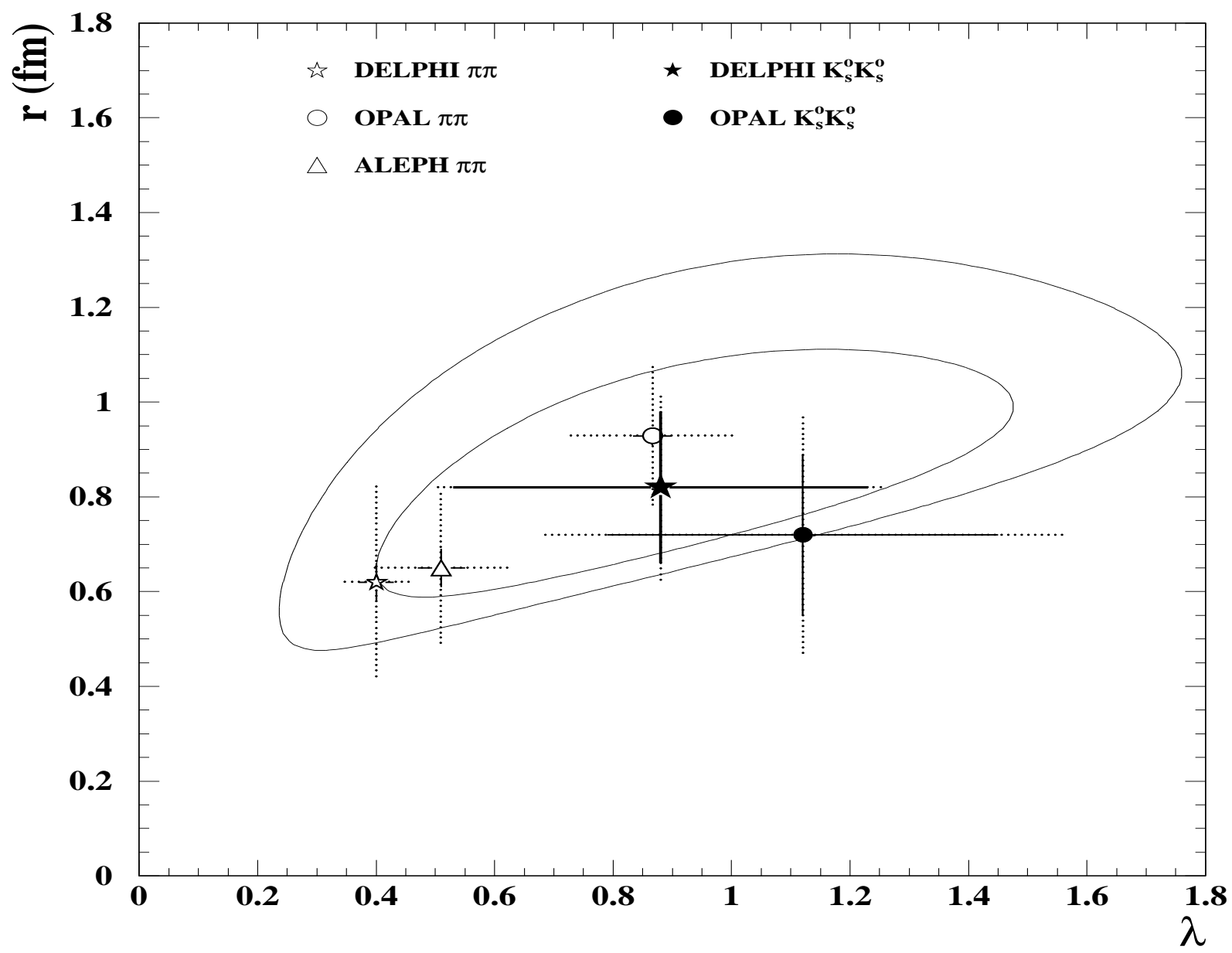

Figure 32: Compilation of fit results from the study of Bose-Einstein correlations for charged pions and neutral kaons. The lines represent $68 \%$ and $90 \%$ confidence level contours [210]. 


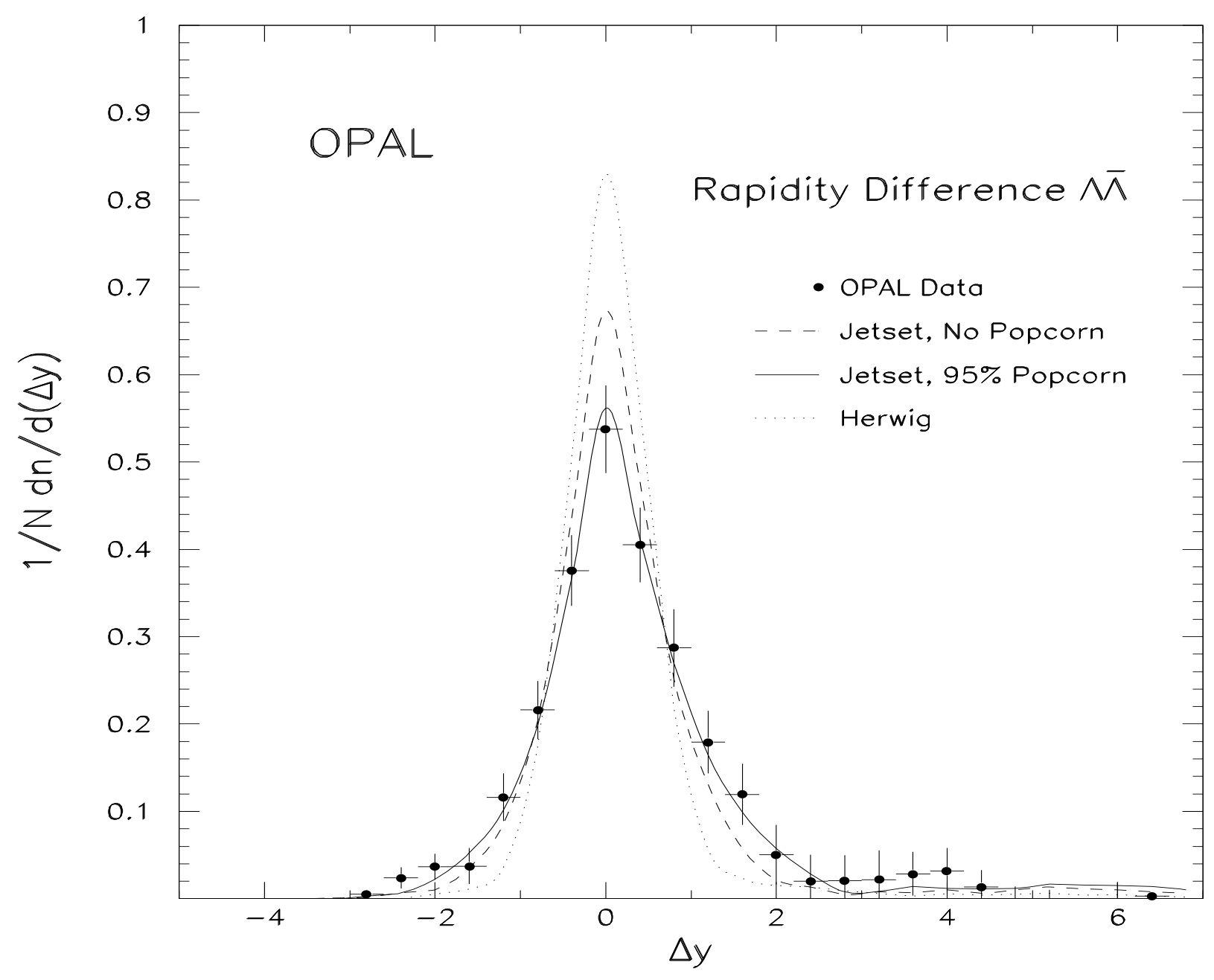

Figure 33: Rapidity difference with respect to the Thrust axis for $\Lambda \bar{\Lambda}$ pairs. The width of the measured distribution [214] compared to the predictions from various Monte-Carlo models supports a string like production mechanism for the final state hadrons. 